\title{
Search for anomalous production of prompt like-sign muon pairs and constraints on physics beyond the standard model with the ATLAS detector
}

\author{
G. Aad et al.* \\ (ATLAS Collaboration) \\ (Received 5 January 2012; published 17 February 2012)
}

\begin{abstract}
An inclusive search for anomalous production of two prompt, isolated muons with the same electric charge is presented. The search is performed in a data sample corresponding to $1.6 \mathrm{fb}^{-1}$ of integrated luminosity collected in 2011 at $\sqrt{s}=7 \mathrm{TeV}$ with the ATLAS detector at the LHC. Muon pairs are selected by requiring two isolated muons of the same electric charge with $p_{\mathrm{T}}>20 \mathrm{GeV}$ and $|\eta|<2.5$. Minimal requirements are placed on the rest of the event activity. The distribution of the invariant mass of the muon pair $m(\mu \mu)$ is found to agree well with the background expectation. Upper limits on the cross section for anomalous production of two muons with the same electric charge are placed as a function of $m(\mu \mu)$ within a fiducial region defined by the event selection. The fiducial cross-section limit constrains the like-sign top-quark pair-production cross section to be below $3.7 \mathrm{pb}$ at $95 \%$ confidence level. The data are also analyzed to search for a narrow like-sign dimuon resonance as predicted for e.g. doubly charged Higgs bosons $\left(H^{ \pm \pm}\right)$. Assuming pair production of $H^{ \pm \pm}$bosons and a branching ratio to muons of $100 \%$ (33\%), this analysis excludes masses below 355 (244) GeV and 251 (209) GeV for $H^{ \pm \pm}$bosons coupling to left-handed and right-handed fermions, respectively.
\end{abstract}

DOI: 10.1103/PhysRevD.85.032004

PACS numbers: $13.85 . \mathrm{Rm}, 12.60 . \mathrm{Cn}, 14.80 . \mathrm{Fd}$

\section{INTRODUCTION}

Events containing two high- $p_{\mathrm{T}}$, prompt, like-sign leptons are rarely produced in the standard model (SM), but occur with an enhanced rate in several models of new physics. For example, supersymmetry [1], universal extra dimensions [2], left-right symmetric models [3-6], Higgs triplet models [7-9], the little Higgs model [10], fourthfamily quarks [11], and flavor-changing neutral currents resulting in the production of like-sign top quarks [12-20] could all give rise to final states with two leptons of the same electric charge. Most of these models would result in an excess of like-sign dimuons over the background with no distinct kinematic features. However, doubly charged Higgs bosons $\left(H^{ \pm \pm}\right)$, predicted by some of those models, would be observed as a narrow resonance in the dimuon mass spectrum.

In the analysis described in this article, events containing like-sign muon pairs are selected and their invariant mass distribution is compared to the SM prediction. Both muons are required to have transverse momentum $p_{\mathrm{T}}>$ $20 \mathrm{GeV}$ and pseudorapidity [21] $|\eta|<2.5$, and they must be isolated from other activity in the event. Upper limits on the cross section of non-SM physics in a fiducial region corresponding to the experimental requirements are derived as a function of the dimuon invariant mass. Results

*Full author list given at the end of the article.

Published by the American Physical Society under the terms of the Creative Commons Attribution 3.0 License. Further distribution of this work must maintain attribution to the author(s) and the published article's title, journal citation, and DOI. are presented inclusively for $\mu^{ \pm} \mu^{ \pm}$production and separately for $\mu^{+} \mu^{+}$and $\mu^{-} \mu^{-}$final states. The $\mu^{+} \mu^{+}$result is further used to constrain like-sign top-quark pair production. The data are also used to search for a narrow dimuon resonance with a width much smaller than the detector resolution of $\sim 3 \%$. An example of a particle that may result in a narrow mass peak is a short-lived $H^{ \pm \pm}$boson, predicted by a number of the models for new physics mentioned above. Constraints on the $H^{ \pm \pm}$ mass as a function of its branching ratio to two muons are presented.

The ATLAS Collaboration has previously reported an inclusive search for new physics in the like-sign dilepton final state in a data sample corresponding to an integrated luminosity of $34 \mathrm{pb}^{-1}$ [22]. No significant deviation from SM expectations was observed, and fiducial cross-section limits as well as limits on several specific models of physics beyond the SM were derived. The CDF Collaboration has performed similar inclusive searches $[23,24]$ without observing any evidence for new physics. Like-sign top-quark pair production has previously been searched for by the CDF [25] and the CMS Collaborations [26]. The upper limit on the cross section set by the CMS Collaboration in $p p$ collisions at $\sqrt{s}=7 \mathrm{TeV}$ is $17 \mathrm{pb}$. Direct limits on $H^{ \pm \pm}$bosons have previously been set at hadron colliders by the CDF [24,27] and D0 [28,29] Collaborations. The most stringent limits to date for $H^{ \pm \pm}$ bosons decaying to dimuons with a branching ratio of $100 \%$ exclude masses below 205-245 GeV depending on the couplings [24].

This article is organized as follows. A brief description of the ATLAS detector is given in Sec. II. Sec. III presents the data and simulation samples used. The event selection 
is described in Sec. IV. The backgrounds are discussed in Sec. V, and Sec. VI summarizes the systematic uncertainties. The data are compared to the background estimate in Sec. VII. The interpretation of the data as a cross-section upper limit within the fiducial region, for four ranges of dimuon invariant mass, and its implication on like-sign top-quark pair production are reported in Secs. VIII and IX, respectively. The narrow resonance search and its interpretation in terms of $H^{ \pm \pm}$boson production is presented in Sec. X. Finally, Sec. XI summarizes the conclusions.

\section{THE ATLAS DETECTOR}

The ATLAS detector [30] consists of an inner tracking system, calorimeters, and a muon spectrometer. The inner detector, directly surrounding the interaction point, is composed of a silicon pixel detector, a silicon strip detector, and a transition radiation tracker, all embedded in a $2 \mathrm{~T}$ axial magnetic field. It covers the pseudorapidity range $|\eta|<2.5$ and is enclosed by a calorimeter system containing electromagnetic and hadronic sections. The calorimeter system is surrounded by a large muon spectrometer built with three air-core toroids. This spectrometer is equipped with precision chambers (composed of monitored drift tubes and cathode strip chambers) to provide precise position measurements in the bending plane in the range $|\eta|<2.7$. In addition, resistive plate chambers and thin gap chambers with a fast response time are used primarily to trigger muons in the rapidity ranges $|\eta| \leq$ 1.05 and $1.05<|\eta|<2.4$, respectively. Momentum measurements in the muon spectrometer are based on track segments formed in at least two of the three precision chambers. The resistive plate chambers and thin gap chambers provide position measurements in the nonbending plane which is used to improve the pattern recognition and the track reconstruction.

The ATLAS detector has a three-level trigger system [31] which reduces the event rate to approximately $200 \mathrm{~Hz}$ before data transfer to mass storage. The Level-1 muon trigger searches for hit coincidences between different muon trigger detector layers inside programmed geometrical windows that define the muon transverse momentum and provide a rough estimate of its position. It selects muons in the rapidity range $|\eta|<2.4$. The Level-1 trigger is followed by a high-level, software-based trigger selection which is similar to that of the offline reconstruction.

\section{DATA SAMPLE AND MONTE CARLO SIMULATION}

This analysis is carried out using a data sample corresponding to an integrated luminosity of $1.6 \mathrm{fb}^{-1}$ recorded between March and July of 2011 at a center-of-mass energy of $7 \mathrm{TeV}$. The data are selected using single-muon triggers with a $p_{\mathrm{T}}$ threshold of $10 \mathrm{GeV}$ at Level-1. At the high-level trigger, a muon with $p_{\mathrm{T}}>18 \mathrm{GeV}$ is required. In this data set, the average number of interactions per beam crossing is about six.

Monte Carlo (MC) simulation is used to estimate some of the background contributions and to determine the selection efficiency and acceptance for possible new physics signals. The dominant SM processes that contribute to prompt likesign dimuon production are $W Z, Z Z, W^{ \pm} W^{ \pm}$, and $t \bar{t} W$. These are all estimated using MC simulation. For processes with a $Z$ boson, the contribution from $\gamma^{*}$ is also simulated for $m(\ell \ell)>20 \mathrm{GeV}$. $W Z$ and $Z Z$ events are generated using HERWIG [32], and $W^{ \pm} W^{ \pm}$and $t \bar{t} W$ production is generated with MADGRAPH [33] for the matrix element and PYTHIA [34] for the parton shower and fragmentation.

The normalization of the $W Z$ and $Z Z$ MC samples is based on cross sections determined at next-to-leadingorder (NLO) using MCFM [35]. The NLO cross sections times branching ratios for $W^{ \pm} Z \rightarrow \ell^{ \pm} \nu \ell^{ \pm} \ell^{\mp}$ and $Z Z \rightarrow$ $\ell^{ \pm} \ell^{\mp} \ell^{ \pm} \ell^{\mp}$, where $\ell^{ \pm}$is an electron, muon, or tau lepton, after requiring two charged leptons with the same electric charge and with $p_{\mathrm{T}}>20 \mathrm{GeV}$ and $|\eta|<2.5$, are $347 \mathrm{fb}$ and $54 \mathrm{fb}$, respectively. The $K$ factors for $W Z$ and $Z Z$ production, defined as the ratios between the NLO and the leading order (LO) cross sections, depend on the kinematic requirements placed on the muons and the invariant mass of the like-sign muon pair. Therefore, $K$-factors that depend on this invariant mass are applied.

Opposite-sign dimuon events due to Drell-Yan, $t \bar{t}$, and $W^{ \pm} W^{\mp}$ production constitute a background if the charge of one of the muons is misidentified. $W^{ \pm} W^{\mp}$ production is generated using HERWIG. The Drell-Yan process is generated with ALPGEN [36], whereas the $t \bar{t}$ background is modeled using MC@NLO [37].

In addition, a variety of new physics signals are simulated in order to study the efficiency and acceptance of the selection cuts.

Like-sign top-quark pair production can occur in models with flavor-changing neutral currents, e.g. via a $t$-channel exchange of a $Z^{\prime}$ boson with $u t Z^{\prime}$ coupling. Since the lefthanded coupling is highly constrained by $B_{d}^{0}-\bar{B}_{d}^{0}$ mixing [38], only right-handed top quarks $\left(t_{R}\right)$ are considered. Samples for this process are produced with the PROTOS [39] generator, using $Z^{\prime}$ mass values of 100,150 and $200 \mathrm{GeV}$. An additional sample is generated, based on an effective four-fermion operator $u u \rightarrow t t$ corresponding to $Z^{\prime}$ masses $\gg 1 \mathrm{TeV}$ [18]. The parton shower and hadronization are performed with PYTHIA.

Pair production of doubly charged Higgs bosons $\left(p p \rightarrow H^{ \pm \pm} H^{\mp \mp}\right.$ ) via a virtual $Z / \gamma^{*}$ exchange is generated using PYTHIA for $H^{ \pm \pm}$mass values between 100 and $400 \mathrm{GeV}$ [40].

Production of a right-handed $W$ boson $\left(W_{R}\right)$ decaying to a charged lepton and a Majorana neutrino $\left(N_{R}\right)$ [41,42], and pair production of heavy down-type fourth generation quarks $\left(d_{4}\right)$ decaying to $t W$ are generated using PYTHIA. 
Parton distribution functions taken from CTEQ6L1 [43] are used for the LO MC generators, while for the $t \bar{t}$ MC@NLO sample CTEQ6.6 [44] parton distribution functions are used.

The detector response to the generated events is simulated with the ATLAS simulation framework [45] using GEANT4 [46], and the events are reconstructed with the same software used to process the data. The simulated response is corrected for the small differences in efficiencies, momentum scales, and momentum resolutions between data and simulation.

\section{EVENT SELECTION}

Events are selected with an inclusive single-muon trigger with a $p_{\mathrm{T}}$ threshold of $18 \mathrm{GeV}$ as described in Sec. III. They must further contain at least two muons of the same electric charge with $p_{\mathrm{T}}>20 \mathrm{GeV}$ and $|\eta|<2.5$. The efficiency of the trigger selection for muon pairs in $Z \rightarrow$ $\mu^{+} \mu^{-}$events passing the event selection used here is $97 \%$. Any combination of two muons is considered, allowing more than one muon pair per event to be included. The invariant mass of the two muons, $m(\mu \mu)$, is required to be larger than $15 \mathrm{GeV}$ to exclude the low-mass hadronic resonances such as the $J / \psi$ and $Y$ mesons. All events used in this analysis are required to have a primary vertex determined with at least five tracks with $p_{\mathrm{T}}>0.4 \mathrm{GeV}$. If more than one interaction vertex is found, the vertex with the highest $\sum_{i=1}^{N} p_{\mathrm{T}, i}^{2}$, where $N$ is the number of tracks associated to the vertex, is defined as the primary vertex.

Muons selected for this analysis are formed from tracks reconstructed in the inner detector combined with tracks reconstructed in the muon spectrometer [47]. The independent charge measurements from these two detectors are required to agree to reduce the charge mismeasurement rate. In addition, the transverse and longitudinal impact parameters with respect to the primary event vertex must be small, $\left|d_{0}\right|<0.2 \mathrm{~mm}$ and $\left|z_{0} \sin \theta\right|<5.0 \mathrm{~mm}$, and the transverse impact parameter significance, $\left|d_{0}\right| / \sigma\left(d_{0}\right)$, is required to be less than 3.0. The muon isolation ( $p_{\mathrm{T}}^{\text {cone40 }}$ ) is defined as the scalar sum of the transverse momenta of all tracks with $p_{\mathrm{T}}>0.5 \mathrm{GeV}$ within a cone around the muon axis of size $\Delta R=\sqrt{\Delta \phi^{2}+\Delta \eta^{2}}=0.4$ that are within $\left|z_{0}\right|<1 \mathrm{~cm}$ of the primary event vertex. Requirements of $p_{\mathrm{T}}^{\text {cone } 40}<5 \mathrm{GeV}$ and $p_{\mathrm{T}}^{\text {cone } 40} / p_{\mathrm{T}}(\mu)<$ 0.08 are made.

The above selection cuts are chosen to retain a high efficiency for prompt muons while rejecting a large fraction of nonprompt backgrounds. For muons from $Z$-boson decays, the efficiency of the impact parameter significance and the isolation cuts ranges from $87 \%$ to $97 \%$ depending on $p_{\mathrm{T}}$, while for muons from $b$ - and $c$-hadron decays, the efficiency is about $3.5 \%$. For muons arising from $\tau$ decays in $Z \rightarrow \tau \tau$ events, the efficiency is about $60 \%$.

\section{BACKGROUND DETERMINATION}

The SM backgrounds for like-sign dimuon final states can be divided into background from production of prompt like-sign dimuons, background caused by muons from hadronic decays (nonprompt muons), and background from processes with two prompt opposite-sign muons where the charge of one of the muons is mismeasured.

The dominant SM processes with two prompt leptons of the same electric charge in the final state are $W^{ \pm} Z \rightarrow$ $\ell^{ \pm} \nu \ell^{ \pm} \ell^{\mp}, Z Z \rightarrow \ell^{ \pm} \ell^{\mp} \ell^{ \pm} \ell^{\mp}, W^{ \pm} W^{ \pm} \rightarrow \ell^{ \pm} \nu \ell^{ \pm} \nu$, and $t \bar{t} W \rightarrow \ell^{ \pm} \ell^{ \pm}+X$. Any other SM processes are found to be negligible. The contribution of these processes to the signal region is estimated from MC simulation using the samples described in Sec. III. In these simulated samples, only muons that originate from a $\tau$ lepton, a $W$ boson, or a $Z$ boson are considered prompt. Muons originating from any other sources are discarded in order to avoid doublecounting with the nonprompt muon background that is derived from data.

Background from nonprompt muons may originate from several different sources: semileptonic $b$ - or $c$-hadron decays, muons from pion or kaon decays in flight, and misidentified muons from hadronic showers in the calorimeter which reach the muon spectrometer and are incorrectly matched to a reconstructed inner detector track [48]. The background from nonprompt muons is estimated from data using a matrix method [49]. This method requires knowledge of the probabilities for prompt and nonprompt muons to pass the isolation requirement. The probability for nonprompt muons to pass the isolation cut is determined using muons with $\left|d_{0}\right| / \sigma\left(d_{0}\right)>5$ in dimuon or single-muon samples. These are dominated by semileptonic $b$ - and $c$ hadron decays. The probability is found to be $5 \%$ rather independently of $p_{\mathrm{T}}$ and $\eta$. A systematic uncertainty is derived from a complementary sample where $\left|d_{0}\right| / \sigma\left(d_{0}\right)<3$ is required. In this sample, prompt muons from $W$ or $Z$ decays are suppressed by requiring there to be exactly one muon in the event, the transverse mass [50] of the muon and the missing transverse energy [51] to be below $10 \mathrm{GeV}$, and at least one jet with $p_{\mathrm{T}}>20 \mathrm{GeV}$ to be present. The resulting systematic uncertainty on the probability for nonprompt muons to pass the isolation cut varies between $30 \%$ and $100 \%$ depending on $p_{\mathrm{T}}$. The probability for prompt muons to pass the isolation cut as a function of $p_{\mathrm{T}}$ and $\eta$ is derived from $Z \rightarrow \mu^{+} \mu^{-}$MC events and is cross-checked with data.

Another source of background arises from opposite-sign muon pairs where the charge of one of the two muons is misidentified. This background source is negligible in the relevant mass range as estimated from simulation. The charge misidentification probability is also measured from $Z \rightarrow \mu \mu$ events in data by exploiting the independent charge measurements provided by the inner detector and the muon spectrometer. It is found to be consistent with zero in the relevant $p_{\mathrm{T}}$ range. Based on observing zero 
charge misidentified events in data, a $68 \%$ upper limit is placed on this probability as function of $p_{\mathrm{T}}$, which ranges up to $10 \%$ at $p_{\mathrm{T}}(\mu)=400 \mathrm{GeV}$. This upper limit is applied as a function of $p_{\mathrm{T}}(\mu)$ to opposite-sign prompt muon pairs in the Drell-Yan, $W^{+} W^{-}$, and $t \bar{t}$ MC samples to determine the systematic uncertainty on this background source.

The background estimate is cross-checked in a variety of samples complementary to the signal region. These include like-sign muon pairs where at least one muon fails the $\left|d_{0}\right| / \sigma\left(d_{0}\right)$ cut, like-sign muon pairs where both muons fail the isolation requirement used in the analysis but pass a looser isolation requirement, like-sign and opposite-sign muon pairs where both muons fail the isolation requirement used in the analysis but pass a looser isolation requirement and at least one muon fails the $\left|d_{0}\right| / \sigma\left(d_{0}\right)$ cut, and opposite-sign muon pairs where both muons pass the final analysis requirements. For all control regions, the data are found to agree with the background prediction within the systematic uncertainties, both in overall event yield and in the shape of the dimuon mass distribution.

\section{SYSTEMATIC UNCERTAINTIES}

Uncertainties on the event selection efficiencies and the luminosity affect the predicted yield of signal events as well as those backgrounds that are estimated purely from MC simulation, i.e. $W Z, Z Z, W^{ \pm} W^{ \pm}$, and $t \bar{t} W$ production. The uncertainty on the muon reconstruction efficiency is $\pm 1 \%$ [52]. In addition, the efficiency of the requirements on impact parameter and isolation is observed to be $3 \%$ lower in data than in simulation at the lowest $p_{\mathrm{T}}$ values while for $p_{\mathrm{T}}>30 \mathrm{GeV}$ data and simulation agree typically within $\pm 1 \%$. The resulting uncertainty on the muon pair selection efficiency due to the muon identification efficiency is ${ }_{-1.8}^{+1.0} \%$. The uncertainty on the muon trigger efficiency of $<1 \%$ [52] results in an uncertainty on the selection efficiency of $\pm 0.3 \%$. The uncertainty in the muon momentum scale [53] results in an uncertainty on the dimuon pair selection efficiency of $\pm 0.9 \%$ due to the migrations across the $p_{\mathrm{T}}$ and $m(\mu \mu)$ cut thresholds. In addition, the integrated luminosity measurement has an uncertainty of $\pm 3.7 \%[54,55]$.

The uncertainty in the production cross sections of the SM processes affect the predicted yield of the prompt muon background. The $W Z$ and $Z Z$ cross-section uncertainties due to higher-order corrections are estimated to be $\pm 10 \%$ by varying the renormalization and factorization scales by a factor of 2 . For $t \bar{t} W$ production, the higher-order corrections are estimated to be similar to those for $t \bar{t} Z$, which are calculated in Ref. [56], and the cross section is taken to be a factor of $1.30 \pm 0.65$ higher than the LO cross section [57]. The full higher-order corrections for $W^{ \pm} W^{ \pm}$ production have not yet been calculated. However, for parts of the process, the NLO QCD corrections have

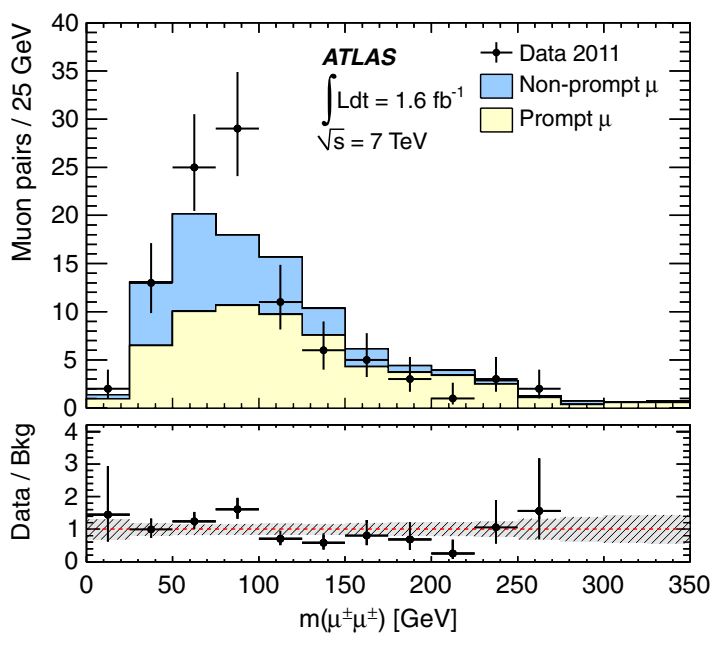

(a)

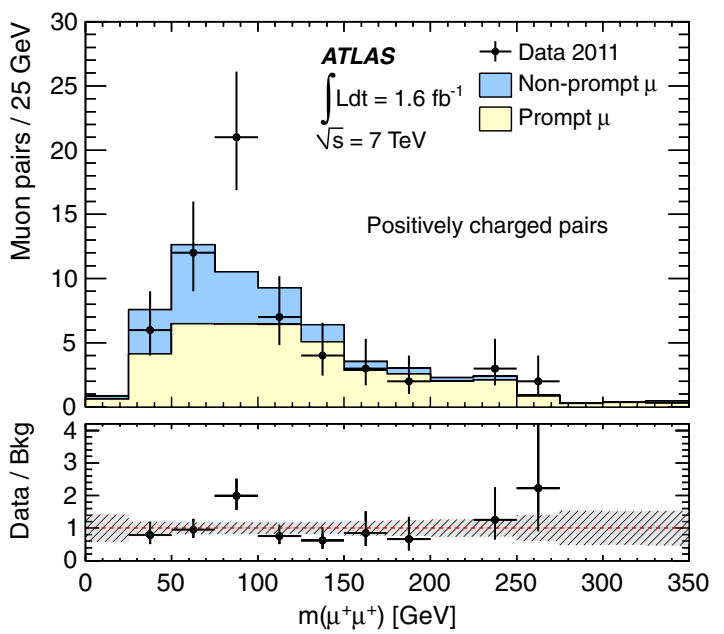

(b)

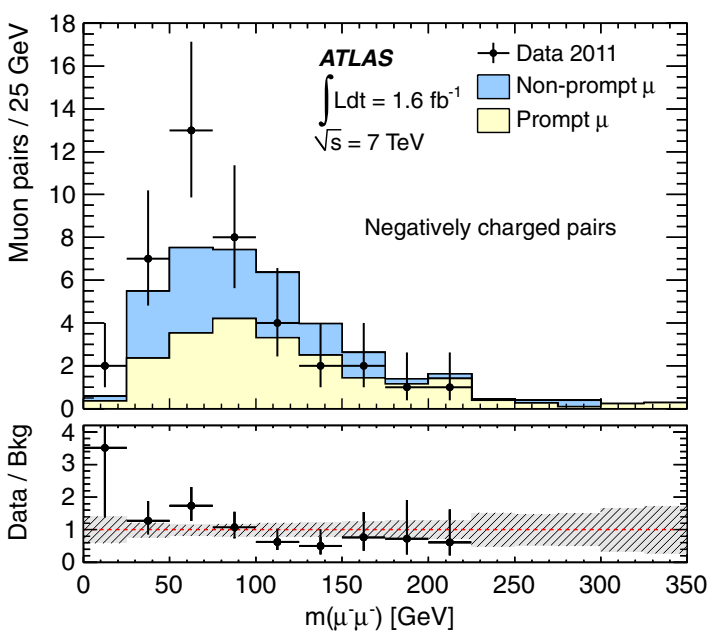

(c)

FIG. 1 (color online). Distribution of the dimuon invariant mass for (a) $\mu^{ \pm} \mu^{ \pm}$pairs, (b) $\mu^{+} \mu^{+}$pairs, and (c) $\mu^{-} \mu^{-}$pairs. The data are compared to the stacked background estimates. The ratio between the data and the predicted background is also shown, where the shaded region is the total systematic uncertainty on the background prediction. 
been shown to be small [58]. Here, the LO cross section is used and an uncertainty of $\pm 50 \%$ is assumed.

Uncertainties on the parton distribution functions affect both the acceptance and the normalization of the prompt muon backgrounds and the new physics models constrained in this paper. This uncertainty is evaluated using the eigenvectors provided by the MSTW20081o68cl set [59] of parton distribution functions using the prescription given in Ref. [60] and adding in quadrature the difference between the central cross-section value obtained using this set and that obtained with the CTEQ6L1 [43] parton distribution functions. For the diboson background, the resulting uncertainty on the cross section is $\pm 7 \%$. The uncertainty on the acceptance due to this source is typically $\pm 2 \%$.

The uncertainty on the number of muon pairs from nonprompt muon backgrounds has systematic and statistical components which are added in quadrature to give the total uncertainty on this background source. The systematic component is derived from the uncertainty on the measurement of the fraction of nonprompt muons passing the isolation cuts which ranges from $\pm 30 \%$ for $m(\mu \mu)>$ $15 \mathrm{GeV}$ to $\pm 80 \%$ for $m(\mu \mu)>300 \mathrm{GeV}$ (see Sec. V).
The statistical component arises from the limited number of nonisolated muons used in the matrix method: this is $\pm 3 \%$ for $m(\mu \mu)>15 \mathrm{GeV}$ and $\pm 45 \%$ for $m(\mu \mu)>$ $300 \mathrm{GeV}$. The background due to charge misidentification has an uncertainty of +2.7 events for the full sample and +0.6 events in the highest mass region.

Any statistical uncertainties due to limited size of the background and signal MC samples are also considered.

Systematic uncertainties on different processes from the same origin are assumed to be $100 \%$ correlated.

\section{COMPARISON OF THE DATA TO THE BACKGROUND EXPECTATION}

The invariant mass distributions observed in the data are compared to the predicted background for $\mu^{ \pm} \mu^{ \pm}, \mu^{+} \mu^{+}$, and $\mu^{-} \mu^{-}$production in Fig. 1 .

Table I summarizes the number of observed and expected muon pairs for $\mu^{ \pm} \mu^{ \pm}, \mu^{+} \mu^{+}$, and $\mu^{-} \mu^{-}$production for four cuts on the dimuon invariant mass. The data agree with the background within the systematic uncertainties and no excess is observed. The number of data events in high-mass bins is lower than the background

TABLE I. Expected and observed numbers of pairs of isolated like-sign muons for various cuts on the dimuon invariant mass, $m(\mu \mu)$. The uncertainties shown are the quadratic sum of the statistical and systematic uncertainties. The prompt muon background contribution includes the $W Z, Z Z, W^{ \pm} W^{ \pm}$, and $t \bar{t} W$ processes.

\begin{tabular}{|c|c|c|c|c|}
\hline \multirow[t]{2}{*}{ Sample } & \multicolumn{4}{|c|}{ Number of muon pairs with $m\left(\mu^{ \pm} \mu^{ \pm}\right)$} \\
\hline & $>15 \mathrm{GeV}$ & $>100 \mathrm{GeV}$ & $>200 \mathrm{GeV}$ & $>300 \mathrm{GeV}$ \\
\hline Prompt muons & $63.1 \pm 7.8$ & $34.9 \pm 4.5$ & $9.6 \pm 1.6$ & $2.24 \pm 0.54$ \\
\hline Nonprompt muons & $37.5_{-12.4}^{+10.3}$ & $13.0 \pm 4.5$ & $1.8 \pm 0.7$ & $0.31 \pm 0.18$ \\
\hline Charge flip & $0_{-0.0}^{+2.7}$ & $0_{-0.0}^{+0.9}$ & $0_{-0.0}^{+0.7}$ & $0_{-0.00}^{+0.61}$ \\
\hline Total & $100.6_{-14.7}^{+13.2}$ & $48.0 \pm 6.4$ & $11.4_{-1.7}^{+1.8}$ & $2.56_{-0.57}^{+0.83}$ \\
\hline Data & 101 & 32 & 7 & 1 \\
\hline \multirow[t]{2}{*}{ Sample } & \multicolumn{4}{|c|}{ Number of muon pairs with $m\left(\mu^{+} \mu^{+}\right)$} \\
\hline & $>15 \mathrm{GeV}$ & $>100 \mathrm{GeV}$ & $>200 \mathrm{GeV}$ & $>300 \mathrm{GeV}$ \\
\hline Prompt muons & $41.2 \pm 5.3$ & $23.5 \pm 3.2$ & $6.6 \pm 1.2$ & $1.33 \pm 0.40$ \\
\hline Nonprompt muons & $20.2_{-6.9}^{+5.9}$ & $6.3 \pm 2.2$ & $1.0 \pm 0.4$ & $0.24 \pm 0.15$ \\
\hline Charge flip & $0_{-0.0}^{+1.3}$ & $0_{-0.0}^{+0.5}$ & $0_{-0.0}^{+0.3}$ & $0_{-0.00}^{+0.30}$ \\
\hline Total & $61.4_{-8.7}^{+8.0}$ & $29.8 \pm 3.9$ & $7.5 \pm 1.3$ & $1.57_{-0.42}^{+0.52}$ \\
\hline Data & 61 & 22 & 6 & 1 \\
\hline \multirow[t]{2}{*}{ Sample } & \multicolumn{4}{|c|}{ Number of muon pairs with $m\left(\mu^{-} \mu^{-}\right)$} \\
\hline & $>15 \mathrm{GeV}$ & $>100 \mathrm{GeV}$ & $>200 \mathrm{GeV}$ & $>300 \mathrm{GeV}$ \\
\hline Prompt muons & $21.9 \pm 3.0$ & $11.4 \pm 1.8$ & $3.04 \pm 0.67$ & $0.91 \pm 0.32$ \\
\hline Nonprompt muons & $17.4_{-5.8}^{+4.7}$ & $6.8 \pm 2.4$ & $0.83 \pm 0.38$ & $0.07_{-0.07}^{+0.08}$ \\
\hline Charge flip & $0_{-0.0}^{+1.3}$ & $0_{-0.0}^{+0.5}$ & $0_{-0.0}^{+0.34}$ & $0_{-0.00}^{+0.30}$ \\
\hline Total & $39.3_{-6.5}^{+5.8}$ & $18.2 \pm 3.0$ & $3.87_{-0.77}^{+0.84}$ & $0.98_{-0.33}^{+0.45}$ \\
\hline Data & 40 & 10 & 1 & 0 \\
\hline
\end{tabular}


expectation, but in all mass bins the probability that the background gives a fluctuation as low or lower than observed in the data is found to be greater than 5\%. In all mass bins, prompt muons from diboson production are the dominant background but nonprompt muons also contribute significantly: about $40 \%$ at low mass and $10 \%$ at high mass.

\section{UPPER LIMITS ON THE CROSS SECTION FOR PROMPT LIKE-SIGN DIMUON PRODUCTION}

A $95 \%$ confidence level (C.L.) upper limit on the number of like-sign muon pairs due to anomalous production, $N_{95}(\mu \mu)$, is obtained using a Bayesian approach with a flat prior for the number of events from new physics, integrating over Gaussian priors for the systematic uncertainties $[61,62]$. All systematic uncertainties discussed above are included, and correlations between their effects on signal and background processes are taken into account.

The upper limit on the number of anomalously produced muon pairs, $N_{95}(\mu \mu)$, ranges from 41 pairs for $m(\mu \mu)>$ $15 \mathrm{GeV}$ to 3.8 pairs for $m(\mu \mu)>300 \mathrm{GeV}$ at $95 \%$ C.L. The limit on the number of muon pairs is translated to a 95\% C.L. limit on the cross section measured in the phase space region defined by the fiducial cuts as

$$
\sigma_{95}^{\mathrm{fid}}(\mu \mu)=\frac{N_{95}(\mu \mu)}{\varepsilon_{\mathrm{fid}} \int \mathcal{L} d t},
$$

where $\int \mathcal{L} d t$ is the integrated luminosity of $1.61 \pm$ $0.06 \mathrm{fb}^{-1}$. The efficiency of the experimental cuts with respect to the fiducial region, $\varepsilon_{\text {fid }}$, depends on the model of new physics. The fiducial cuts used to define the efficiency are closely matched to those imposed at reconstruction level: both muons must have $p_{\mathrm{T}}>20 \mathrm{GeV},|\eta|<2.5$, and be separated by $\Delta R>0.4$ from any jet or prompt muon or electron with $p_{\mathrm{T}}>20 \mathrm{GeV}$.

A variety of models is considered for the determination of $\varepsilon_{\text {fid }}$, and the lowest efficiency value obtained among all the models is used. The models considered are like-sign top-quark pair production via an effective four-fermion coupling, Majorana neutrino $\left(N_{R}\right)$ production from the decay of a $W_{R}$ boson, pair production of fourth generation quarks decaying via top quarks, and doubly charged Higgs boson production. A variety of mass values for those models is considered: $800 \leq m\left(W_{R}\right) \leq 1500 \mathrm{GeV}$ and $100 \leq m\left(N_{R}\right) \leq 1300 \mathrm{GeV}, \quad 300 \leq m\left(d_{4}\right) \leq 500 \mathrm{GeV}$, and $100 \leq m\left(H^{ \pm \pm}\right) \leq 300 \mathrm{GeV}$. The efficiency values obtained from any of these samples with respect to the fiducial cuts vary for different models and mass bins due primarily to the $p_{\mathrm{T}}$ dependence of the isolation efficiency. Like-sign top-quark pair production results in the lowest fiducial efficiency of $43.9_{-2.4}^{+1.9} \%$ for $m\left(\mu^{ \pm} \mu^{ \pm}\right)>$ $300 \mathrm{GeV}$, while a model with $W_{R}$ boson of $800 \mathrm{GeV}$ decaying to a $500 \mathrm{GeV}$ Majorana neutrino gives the highest value of $72.5_{-2.2}^{+1.6 \%}$. For pair production of $100 \mathrm{GeV} H^{ \pm \pm}$
TABLE II. Expected and observed 95\% C.L. upper limit on the cross section, $\sigma_{95}^{\mathrm{fid}}$, for new physics in bins of dimuon mass for like-sign muon pairs with $p_{\mathrm{T}}(\mu)>20 \mathrm{GeV},|\eta(\mu)|<2.5$, and $\Delta R>0.4$ between the muon and any jet, prompt electron or prompt muon with $p_{T}>20 \mathrm{GeV}$.

\begin{tabular}{lcc}
\hline \hline Mass range $[\mathrm{GeV}]$ & \multicolumn{2}{c}{$\sigma_{95}^{\mathrm{fid}}[\mathrm{fb}]$} \\
& Expected & Observed \\
\hline All muon pairs & $58_{-17}^{+19}$ & 58 \\
$m\left(\mu^{ \pm} \mu^{ \pm}\right)>15$ & $30_{-9}^{+11}$ & 16 \\
$m\left(\mu^{ \pm} \mu^{ \pm}\right)>100$ & $13.7_{-4.4}^{+5.7}$ & 8.4 \\
$m\left(\mu^{ \pm} \mu^{ \pm}\right)>200$ & $8.0_{-2.6}^{+3.3}$ & 5.3 \\
$m\left(\mu^{ \pm} \mu^{ \pm}\right)>300$ & & \\
\hline Positively charged muon pairs & $37_{-11}^{+14}$ & 37 \\
$m\left(\mu^{+} \mu^{+}\right)>15$ & $21.8_{-6.9}^{+9.1}$ & 14.1 \\
$m\left(\mu^{+} \mu^{+}\right)>100$ & $10.3_{-2.2}^{+5.7}$ & 9.1 \\
$m\left(\mu^{+} \mu^{+}\right)>200$ & $7.2_{-2.9}^{+1.8}$ & 5.6 \\
$m\left(\mu^{+} \mu^{+}\right)>300$ & & \\
\hline Negatively charged muon pairs & $29_{-8}^{+11}$ & 30 \\
$m\left(\mu^{-} \mu^{-}\right)>15$ & $17.0_{-5.1}^{+6.5}$ & 9.5 \\
$m\left(\mu^{-} \mu^{-}\right)>100$ & $8.7_{-2.5}^{+3.1}$ & 5.2 \\
$m\left(\mu^{-} \mu^{-}\right)>200$ & $5.9_{-1.6}^{+1.8}$ & 4.3 \\
$m\left(\mu^{-} \mu^{-}\right)>300$ & & \\
\hline \hline
\end{tabular}

bosons, the fiducial efficiency is $69.8_{-2.0}^{+1.5} \%$ for $m\left(\mu^{ \pm} \mu^{ \pm}\right)>15 \mathrm{GeV}$. The efficiency uncertainties include all sources discussed in Sec. VI. To derive the cross-section limits, the lowest efficiency value of $43.9_{-2.4}^{+1.9} \%$ is used in all mass bins. The resulting limits are given in Table II for the four mass ranges and separately for $\mu^{ \pm} \mu^{ \pm}, \mu^{+} \mu^{+}$, and $\mu^{-} \mu^{-}$production.

\section{LIMITS ON LIKE-SIGN TOP-QUARK PAIR PRODUCTION}

Like-sign top-quark pair production can occur if e.g. a flavor-changing $Z^{\prime}$ boson that couples to $u$ and $t$ quarks is exchanged in the $t$ channel. The fiducial cross-section limits presented above are used to constrain this model.

In order to assess the impact on any physics model, the acceptance of the fiducial cuts with respect to the full phase space, $A_{\text {fid }}$, needs to be determined. The cross-section limit for that model is then given by

$$
\sigma_{95}=\frac{\sigma_{95}^{\mathrm{fid}}(\mu \mu)}{A_{\mathrm{fid}}} .
$$

For the model of like-sign top-quark production, only $\mu^{+} \mu^{+}$pairs are considered since the $\mu^{-} \mu^{-}$process contributes less than $3 \%$ at the LHC due to the much smaller $\bar{u}$-quark density compared to the $u$-quark density in the proton. The fiducial acceptance for the production of 
TABLE III. Upper limit at 95\% C.L. on the $t_{R} t_{R}$ production cross section, $\sigma_{95}\left(t_{R} t_{R}\right)$, for four $Z^{\prime}$ mass values based on the $\mu^{+} \mu^{+}$search with $m\left(\mu^{+} \mu^{+}\right)>200 \mathrm{GeV}$.

\begin{tabular}{lcc}
\hline \hline$m\left(Z^{\prime}\right)$ & \multicolumn{2}{c}{$\sigma_{95}\left(t_{R} t_{R}\right)[\mathrm{pb}]$} \\
& Expected & Observed \\
\hline $100 \mathrm{GeV}$ & $4.2_{-0.9}^{+2.3}$ & 3.7 \\
$150 \mathrm{GeV}$ & $3.3_{-0.7}^{+1.9}$ & 3.0 \\
$200 \mathrm{GeV}$ & $2.9_{-0.6}^{+1.6}$ & 2.6 \\
$\gg 1 \mathrm{TeV}$ & $2.5_{-0.5}^{+1.4}$ & 2.2 \\
\hline \hline
\end{tabular}

right-handed like-sign top quarks, $A_{\text {fid }}\left(t_{R} t_{R}\right)$, is determined for each mass cut and for four $Z^{\prime}$ mass values. For $m\left(Z^{\prime}\right)=$ $100 \mathrm{GeV}\left(m\left(Z^{\prime}\right) \gg 1 \mathrm{TeV}\right), A_{\text {fid }}$ ranges from $0.69 \%$ $(0.62 \%)$ for $m\left(\mu^{+} \mu^{+}\right)>15 \mathrm{GeV}$ to $0.12 \%$ (0.29\%) for $m\left(\mu^{+} \mu^{+}\right)>300 \mathrm{GeV}$. This acceptance is defined with respect to inclusive decays of the $W$ bosons, so the small values are primarily caused by the low $W \rightarrow \mu \nu$ branching ratio. The relative uncertainty on the acceptance is typically $2-3 \%$ and accounts for both the statistical uncertainty and the uncertainty due to the parton distribution functions as discussed in Sec. VI.

The mass range that gives the best expected limits is $m\left(\mu^{+} \mu^{+}\right)>200 \mathrm{GeV}$ for all $m\left(Z^{\prime}\right)$. The results are listed in Table III for four $Z^{\prime}$ masses. The upper limits on the $t_{R} t_{R}$ production cross section range from 2.2 to $3.7 \mathrm{pb}$ depending on $m\left(Z^{\prime}\right)$.

\section{CONSTRAINTS ON DOUBLY CHARGED HIGGS BOSONS}

The data are used to constrain the production of a narrow resonance decaying to two muons, using as reference model the production of $H^{ \pm \pm}$bosons. In Sec. X A the model considered for $H^{ \pm \pm}$production is described and the results are presented in Sec. X B.

\section{A. $\boldsymbol{H}^{ \pm \pm}$boson production}

The production process of doubly charged Higgs bosons considered here is pair production via the exchange of a virtual $Z / \gamma^{*}$ [63]. Other production mechanisms may contribute in addition but they depend on other model parameters such as the masses of the neutral and singly charged Higgs bosons and are therefore not included. Only $H^{ \pm \pm}$ bosons decaying to muons with coupling values between $10^{-5}$ and 0.5 are considered to ensure a short lifetime $(c \tau<10 \mu \mathrm{m})$ and that the relative natural width, $\Gamma / M$, is less than 1\%. Doubly charged Higgs bosons couple to Higgs and electroweak gauge bosons and either lefthanded or right-handed charged leptons, and are denoted $H_{L}^{ \pm \pm}$or $H_{R}^{ \pm \pm}$, respectively. While $H_{L}^{ \pm \pm}$couple both to the $Z$ boson and to photons, $H_{R}^{ \pm \pm}$bosons only couple to photons, i.e. coupling to any hypothetical right-handed gauge bosons is neglected, resulting in a 2.5 times smaller pair-production cross section for the latter.
Next-to-leading-order calculations of the $H^{ \pm \pm}$pairproduction cross section via the Drell-Yan process are used [64]. Higher-order QCD corrections beyond the next-to-leading-order accuracy are expected to increase the cross section by about $5 \%$ but are neglected here. The uncertainty on the cross section is $\pm 10 \%$ due to scale dependence in the NLO calculation, parton distribution function uncertainties, and neglected electroweak corrections [65].

\section{B. Constraints on $\boldsymbol{H}^{ \pm \pm}$bosons}

The data are used to derive an upper limit on $H^{ \pm \pm}$pair production via the Drell-Yan process. For this purpose, counting experiments are performed in steps of 10 (20) $\mathrm{GeV}$ for $m(\mu \mu)<200 \mathrm{GeV}(m(\mu \mu) \geq 200 \mathrm{GeV})$ in a mass window of size $\pm 10 \%$ of the central mass, corresponding to about 3 times the experimental mass resolution.

The product of the acceptance and efficiency to detect a single $H^{ \pm \pm}$boson is evaluated based on simulated samples. It is $46 \%$ at $m\left(H^{ \pm \pm}\right)=100 \mathrm{GeV}$ and increases to $57 \%$ at $300 \mathrm{GeV}$. Uncertainties on the acceptance arise from the parton distribution functions, the interpolation between $H^{ \pm \pm}$mass values, and the limited MC statistics. Adding these three uncertainties in quadrature, an overall acceptance uncertainty of $\pm 3.6 \%$ is obtained. The other systematic uncertainties are propagated as described in Sec. VI.

This analysis aims to constrain the pair production ( $p p \rightarrow H^{++} H^{--}$) process. In the analysis, however, likesign muon pairs are counted, and two muon pairs per event can contribute. The cross section for pair production of $H^{ \pm \pm}$bosons, $\sigma_{H H}$, is related to the number of reconstructed dimuon pairs, $N\left(\mu^{ \pm} \mu^{ \pm}\right)$, by

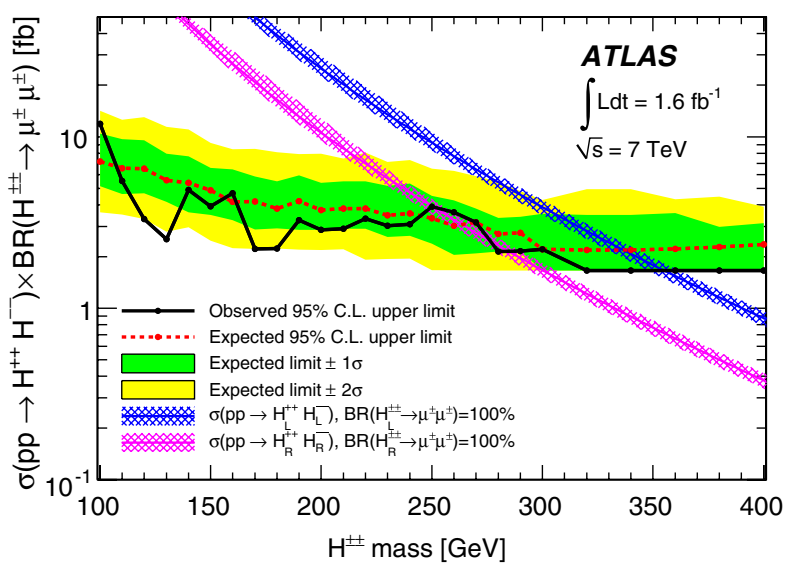

FIG. 2 (color online). Upper limit at 95\% C.L. on the cross section times branching ratio for pair production of doubly charged Higgs bosons decaying to two muons. Superimposed is the predicted cross section for $H_{L}^{++} H_{L}^{--}$and $H_{R}^{++} H_{R}^{--}$ production assuming a branching ratio to muons of $100 \%$. The bands on the predicted cross sections corresponds to the theoretical uncertainty of $10 \%$. 


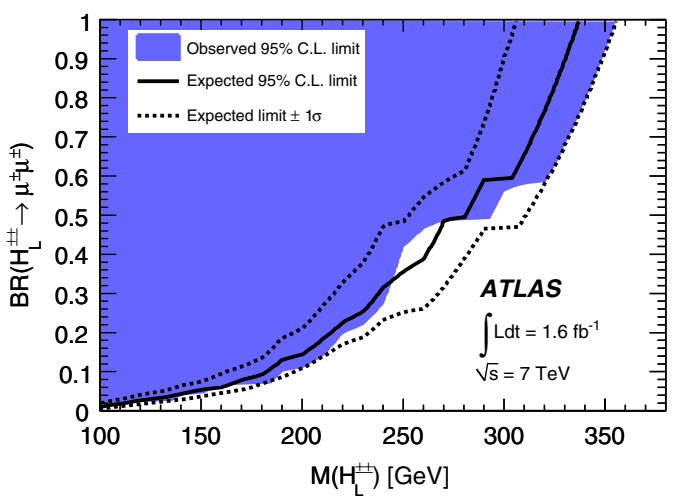

(a)

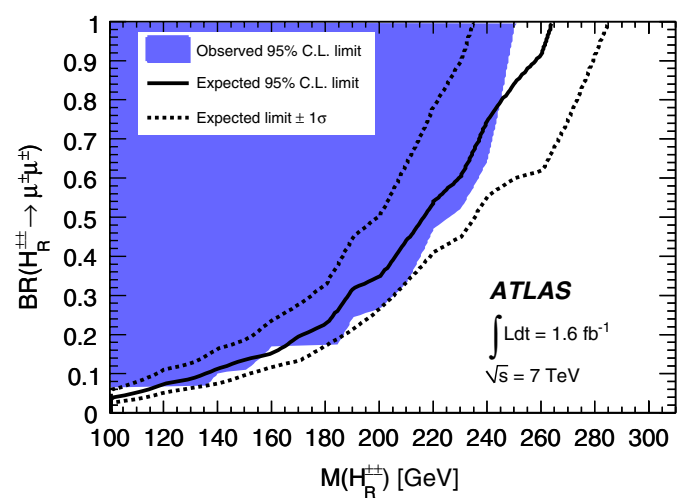

(b)

FIG. 3 (color online). Exclusion region at $95 \%$ C.L. of the $H^{ \pm \pm}$mass as a function of the branching ratio to muon pairs, $B R\left(H^{ \pm \pm} \rightarrow\right.$ $\mu^{ \pm} \mu^{ \pm}$), for a) $H_{L}^{ \pm \pm}$bosons and b) $H_{R}^{ \pm \pm}$bosons. The shaded areas show the observed exclusion region, the solid lines show the expected exclusion region, and the dashed lines show the $\pm 1 \sigma$ variations of the expected exclusion region.

$$
\sigma_{H H} \times B R\left(H^{ \pm \pm} \rightarrow \mu^{ \pm} \mu^{ \pm}\right)=\frac{N\left(\mu^{ \pm} \mu^{ \pm}\right)}{2 \times A \times \epsilon \times \mathcal{L} d t},
$$

where $A \times \epsilon$ is the acceptance times efficiency to detect a single $\mu^{ \pm} \mu^{ \pm}$pair with invariant mass within $10 \%$ of the considered $H^{ \pm \pm}$mass value. It was verified that for this process the efficiency for detecting a single $\mu^{ \pm} \mu^{ \pm}$pair is not affected by the presence of a second pair in the event.

The cross-section limits are obtained using the same procedure as described in Sec. VIII. The expected and observed upper limits at $95 \%$ C.L. on the cross section times the branching ratio, $\sigma\left(p p \rightarrow H^{++} H^{--}\right) \times$ $B R\left(H^{ \pm \pm} \rightarrow \mu^{ \pm} \mu^{ \pm}\right)$, are shown in Fig. 2. The observed upper limit is $11 \mathrm{fb}$ at $m\left(H^{ \pm \pm}\right)=100 \mathrm{GeV}$ and $1.7 \mathrm{fb}$ at $m\left(H^{ \pm \pm}\right)=400 \mathrm{GeV}$. The median expected upper limits based on the background expectation together with the $\pm 1 \sigma$ and $\pm 2 \sigma$ uncertainty bands are also shown. The results derived from data are consistent with the expectation over the full mass range.

The cross-section limit is compared to the prediction for the pair-production cross section of $H_{L}^{ \pm \pm}$and $H_{R}^{ \pm \pm}$bosons, assuming a branching ratio for the dimuon decay of $100 \%$. For this scenario, $H_{L}^{ \pm \pm}$bosons are excluded for $m\left(H_{L}^{ \pm \pm}\right)<$ $355 \mathrm{GeV}$, while $H_{R}^{ \pm \pm}$bosons are excluded for $m\left(H_{R}^{ \pm \pm}\right)<$ $251 \mathrm{GeV}$ at $95 \%$ C.L. for the central value of the theoretical prediction. The corresponding expected limits are $337 \mathrm{GeV}$ and $264 \mathrm{GeV}$, respectively. Using a $10 \%$ lower value for the theoretical prediction (corresponding to the $1 \sigma$ uncertainty on the cross section), the data exclude $m\left(H_{L}^{ \pm \pm}\right)<348 \mathrm{GeV}$ and $m\left(H_{R}^{ \pm \pm}\right)<248 \mathrm{GeV}$.

The observed and expected limits on the mass of doubly charged Higgs bosons are also determined as a function of the branching ratio to $\mu^{ \pm} \mu^{ \pm}$assuming the central value of the theoretical cross-section prediction. This is shown in Fig. 3 for $H_{L}^{ \pm \pm}$and $H_{R}^{ \pm \pm}$bosons, respectively. For example, assuming a branching ratio of $33 \%$ to muons, the respective lower mass limits are $244 \mathrm{GeV}$ for $H_{L}^{ \pm \pm}$and $209 \mathrm{GeV}$ for $H_{R}^{ \pm \pm}$bosons.

\section{CONCLUSIONS}

An inclusive search for production of pairs of prompt like-sign muons has been presented using a data set corresponding to an integrated luminosity of $1.6 \mathrm{fb}^{-1}$ recorded with the ATLAS detector at the LHC. The data agree with the background expectation and no sign of new physics has been found. The data are used to place model-independent upper limits on the cross section of new physics processes giving rise to like-sign dimuons ranging from $5.3 \mathrm{fb}$ for $m\left(\mu^{ \pm} \mu^{ \pm}\right)>300 \mathrm{GeV}$ to $58 \mathrm{fb}$ for $m\left(\mu^{ \pm} \mu^{ \pm}\right)>15 \mathrm{GeV}$. In addition, constraints are placed on like-sign top-quark and doubly charged Higgs boson production. The 95\% C.L. limit on the like-sign top-quark production cross section of $3.7 \mathrm{pb}$ is more than 4 times more restrictive than previous results. The lower mass limit on doubly charged Higgs bosons with a 100\% (33\%) branching ratio to muons is 355 (244) GeV and 251 (209) GeV for $H^{ \pm \pm}$ bosons coupling to left-handed and right-handed fermions, respectively.

\section{ACKNOWLEDGMENTS}

We thank CERN for the very successful operation of the LHC, as well as the support staff from our institutions without whom ATLAS could not be operated efficiently. We also thank Michael Spira for his help with some of the theoretical aspects of the analysis. We acknowledge the support of ANPCyT, Argentina; YerPhI, Armenia; ARC, Australia; BMWF, Austria; ANAS, Azerbaijan; SSTC, Belarus; CNPq and FAPESP, Brazil; NSERC, NRC and CFI, Canada; CERN; CONICYT, Chile; CAS, MOST and NSFC, China; COLCIENCIAS, Colombia; MSMT CR, MPO CR and VSC CR, Czech Republic; DNRF, DNSRC and Lundbeck Foundation, Denmark; ARTEMIS, European Union; IN2P3-CNRS, CEA-DSM/IRFU, France; GNAS, Georgia; BMBF, DFG, HGF, MPG and AvH Foundation, Germany; GSRT, Greece; ISF, MINERVA, GIF, DIP and Benoziyo Center, Israel; INFN, 
Italy; MEXT and JSPS, Japan; CNRST, Morocco; FOM and NWO, Netherlands; RCN, Norway; MNiSW, Poland; GRICES and FCT, Portugal; MERYS (MECTS), Romania; MES of Russia and ROSATOM, Russian Federation; JINR; MSTD, Serbia; MSSR, Slovakia; ARRS and MVZT, Slovenia; DST/NRF, South Africa; MICINN, Spain; SRC and Wallenberg Foundation, Sweden; SER, SNSF and Cantons of Bern and Geneva, Switzerland; NSC, Taiwan; TAEK, Turkey; STFC, the Royal Society and
Leverhulme Trust, United Kingdom; DOE and NSF, United States of America. The crucial computing support from all WLCG partners is acknowledged gratefully, in particular, from CERN and the ATLAS Tier-1 facilities at TRIUMF (Canada), NDGF (Denmark, Norway, Sweden), CC-IN2P3 (France), KIT/GridKA (Germany), INFNCNAF (Italy), NL-T1 (Netherlands), PIC (Spain), ASGC (Taiwan), RAL (UK) and BNL (USA) and in the Tier-2 facilities worldwide.
[1] R. M. Barnett, J. Gunion, and H. Haber, Phys. Lett. B 315, 349 (1993).

[2] J. Alwall, P. Schuster, and N. Toro, Phys. Rev. D 79, 075020 (2009).

[3] J. C. Pati and A. Salam, Phys. Rev. D 10, 275 (1974).

[4] R. N. Mohapatra and J.C. Pati, Phys. Rev. D 11, 566 (1975).

[5] G. Senjanovic and R. N. Mohapatra, Phys. Rev. D 12, 1502 (1975).

[6] T. Rizzo, Phys. Rev. D 25, 1355 (1982); 27, 657 (1983).

[7] J. E. Cieza Montalvo, N. V. Cortez, J. Sa Borges, and M. D. Tonasse, Nucl. Phys. B756, 1 (2006); B796, 422(E) (2008).

[8] H. Georgi and M. Machacek, Nucl. Phys. B262, 463 (1985).

[9] J. F. Gunion, R. Vega, and J. Wudka, Phys. Rev. D 42, 1673 (1990).

[10] N. Arkani-Hamed et al., J. High Energy Phys. 08 (2002) 021.

[11] P. H. Frampton, P. Q. Hung, and M. Sher, Phys. Rep. 330, 263 (2000)

[12] S. Jung, H. Murayama, A. Pierce, and J. D. Wells, Phys. Rev. D 81, 015004 (2010).

[13] J. Cao, Z. Heng, L. Wu, and J. M. Yang, Phys. Rev. D 81, 014016 (2010).

[14] D. Choudhury, R. M. Godbole, S. D. Rindani, and P. Saha, Phys. Rev. D 84, 014023 (2011).

[15] J. Cao, L. Wang, L. Wu, and J. M. Yang, Phys. Rev. D 84, 074001 (2011).

[16] B. Bhattacherjee, S. S. Biswal, and D. Ghosh, Phys. Rev. D 83, 091501 (2011).

[17] E. L. Berger, Q.-H. Cao, C.-R. Chen, C.-S. Li, and H. Zhang, Phys. Rev. Lett. 106, 201801 (2011).

[18] J. Aguilar-Saavedra and M. Perez-Victoria, Phys. Lett. B 701, 93 (2011).

[19] P. Ko, Y. Omura, and C. Yu, arXiv:1108.4005 [J. High Energy Phys. (to be published)].

[20] P. Ko, Y. Omura, and C. Yu, arXiv:1108.0350.

[21] ATLAS uses a right-handed coordinate system with its origin at the nominal interaction point (IP) in the center of the detector and the $z$-axis along the beam pipe. The $x$-axis points from the IP to the center of the LHC ring, and the $y$ axis points upward. Cylindrical coordinates $(r, \phi)$ are used in the transverse plane, $\phi$ being the azimuthal angle around the beam pipe. The pseudorapidity is defined in terms of the polar angle $\theta$ as $\eta=-\ln \tan (\theta / 2)$.

[22] G. Aad et al. (ATLAS Collaboration), J. High Energy Phys. 10 (2011) 107.

[23] CDF Collaboration, Phys. Rev. Lett. 98, 221803 (2007).

[24] CDF Collaboration, Phys. Rev. Lett. 107, 181801 (2011).

[25] CDF Collaboration, CDF Public Note Report No. 10466.

[26] S. Chatrchyan et al. (CMS Collaboration), J. High Energy Phys. 08 (2011) 005.

[27] CDF Collaboration, Phys. Rev. Lett. 93, 221802 (2004).

[28] D0 Collaboration, Phys. Rev. Lett. 101, 071803 (2008).

[29] D0 Collaboration, Phys. Rev. Lett. 108, 021801 (2012).

[30] ATLAS Collaboration, JINST 3, S08003 (2008).

[31] ATLAS Collaboration, Eur. Phys. J. C 72, 1849 (2012).

[32] G. Corcella et al., J. High Energy Phys. 01 (2001) 010.

[33] J. Alwall et al., J. High Energy Phys. 06 (2011) 128.

[34] T. Sjostrand, S. Mrenna, and P. Skands, J. High Energy Phys. 05 (2006) 026.

[35] J.M. Campbell, R. K. Ellis, and C. Williams, J. High Energy Phys. 07 (2011) 018.

[36] M. L. Mangano, M. Moretti, F. Piccinini, R. Pittau, and A. D. Polosa, J. High Energy Phys. 07 (2003) 001.

[37] S. Frixione and B. Webber, J. High Energy Phys. 06 (2002) 029.

[38] Q.-H. Cao, H. McKeen, J. L. Rosner, G. Shaughnessy, and C. E. M. Wagner, Phys. Rev. D 81, 114004 (2010).

[39] J. A. Aguilar-Saavedra, Nucl. Phys. 843, 638 (2011).

[40] V. Rentala, W. Shepherd, and S. Su, Phys. Rev. D 84, 035004 (2011).

[41] F. del Aguila, S. Bar-Shalom, A. Soni, and J. Wudka, Phys. Lett. B 670, 399 (2009).

[42] R. N. Mohapatra and J. C. Pati, Phys. Rev. D 11, 2558 (1975).

[43] J. Pumplin, D. R. Stump, J. Huston, H. L. Lai, P. Nadolsky, and W. K. Tung, J. High Energy Phys. 07 (2002) 012.

[44] P. M. Nadolsky et al. (CTEQ Collaboration), Phys. Rev. D 78, 013004 (2008).

[45] ATLAS Collaboration, Eur. Phys. J. C 70, 823 (2010).

[46] S. Agostinelli et al. (GEANT4 Collaboration), Nucl. Instrum. Methods Phys. Res., Sect. A 506, 250 (2003).

[47] R. Nicolaidou, L. Chevalier, S. Hassani, J. F. Laporte, E. Le Menedeu, and A. Ouraou, J. Phys. Conf. Ser. 219, 032052 (2010).

[48] ATLAS Collaboration, Phys. Lett. B 707, 438 (2012).

[49] ATLAS Collaboration, Eur. Phys. J. C 71, 1577 (2011). 
[50] The transverse mass is defined as $\sqrt{2 E_{\mathrm{T}}^{\text {miss }} p_{\mathrm{T}}(\mu)\left(1-\cos \Delta \phi\left(\mu, E_{\mathrm{T}}^{\text {miss }}\right)\right)}$ where $E_{\mathrm{T}}^{\text {miss }}$ is the missing transverse energy as explained in Ref. [51] and $\Delta \phi\left(\mu, E_{\mathrm{T}}^{\mathrm{miss}}\right)$ is the difference between the azimuthal angles of the muon and $E_{\mathrm{T}}^{\text {miss }}$.

[51] ATLAS Collaboration, Eur. Phys. J. C 72, 1844 (2012).

[52] ATLAS Collaboration, Report No. ATLAS-CONF-2011110, 2011 (unpublished).

[53] ATLAS Collaboration, Report No. ATLAS-CONF-2011046, 2011 (unpublished).

[54] ATLAS Collaboration, Report No. ATLAS-CONF-2011116, 2011 (unpublished).

[55] ATLAS Collaboration, Eur. Phys. J. C 71, 1630 (2011).

[56] A. Lazopoulos, T. McElmurry, K. Melnikov, and F. Petriello, Phys. Lett. B 666, 62 (2008).
[57] F. Petriello (private communication).

[58] B. Jäger, C. Oleari, and D. Zeppenfeld, Phys. Rev. D 80, 034022 (2009).

[59] A. D. Martin, W. J. Stirling, R. S. Thorne, and G. Watt, Eur. Phys. J. C 63, 189 (2009).

[60] J. Campbell, J. Huston, and W. J. Stirling, Rep. Prog. Phys. 70, 89 (2007).

[61] T. Junk, CDF note Report No. 8128, 2007 (unpublished).

[62] J. Heinrich, CDF note Report No. 7587, 2005 (unpublished).

[63] J. F. Gunion, J. Grifols, A. Mendez, B. Kayser, and F. Olness, Phys. Rev. D 40, 1546 (1989); N. G. Deshpande, J. F. Gunion, and B. Kayser, Phys. Rev. D 44, 837 (1991).

[64] M. Mühlleitner and M. Spira, Phys. Rev. D 68, 117701 (2003).

[65] M. Spira (private communication).

G. Aad, ${ }^{47}$ B. Abbott,${ }^{110}$ J. Abdallah, ${ }^{11}$ A. A. Abdelalim, ${ }^{48}$ A. Abdesselam, ${ }^{117}$ O. Abdinov, ${ }^{10}$ B. Abi, ${ }^{111}$ M. Abolins, ${ }^{87}$ O. S. AbouZeid ${ }^{157}$ H. Abramowicz, ${ }^{152}$ H. Abreu ${ }^{114}$ E. Acerbi, ${ }^{88 a, 88 b}$ B. S. Acharya, ${ }^{163 a, 163 b}$ L. Adamczyk,${ }^{37}$ D. L. Adams, ${ }^{24}$ T. N. Addy, ${ }^{55}$ J. Adelman,,${ }^{174}$ M. Aderholz, ${ }^{98}$ S. Adomeit,${ }^{97}$ P. Adragna, ${ }^{74}$ T. Adye, ${ }^{128}$ S. Aefsky, ${ }^{22}$ J. A. Aguilar-Saavedra, ${ }^{123 b, b}$ M. Aharrouche ${ }^{80}$ S. P. Ahlen,${ }^{21}$ F. Ahles ${ }^{47}$ A. Ahmad, ${ }^{147}$ M. Ahsan, ${ }^{40}$

G. Aielli, ${ }^{132 a, 1323 b}$ T. Akdogan, ${ }^{18 a}$ T. P. A. Åkesson, ${ }^{78}$ G. Akimoto, ${ }^{154}$ A. V. Akimov, ${ }^{93}$ A. Akiyama, ${ }^{66}$ M. S. Alam, ${ }^{1}$ M. A. Alam, ${ }^{75}$ J. Albert, ${ }^{168}$ S. Albrand, ${ }^{54}$ M. Aleksa, ${ }^{29}$ I. N. Aleksandrov, ${ }^{64}$ F. Alessandria, ${ }^{88 a}$ C. Alexa, ${ }^{25 a}$ G. Alexander, ${ }^{152}$ G. Alexandre, ${ }^{48}$ T. Alexopoulos, ${ }^{9}$ M. Alhroob,${ }^{20}$ M. Aliev, ${ }^{15}$ G. Alimonti, ${ }^{88 a}$ J. Alison, ${ }^{119}$ M. Aliyev, ${ }^{10}$ P. P. Allport ${ }^{72}$ S. E. Allwood-Spiers,${ }^{52}$ J. Almond, ${ }^{81}$ A. Aloisio, ${ }^{101 a, 101 b}$ R. Alon, ${ }^{170}$ A. Alonso, ${ }^{78}$ B. Alvarez Gonzalez, ${ }^{87}$ M. G. Alviggi, ${ }^{101 \mathrm{a}, 101 \mathrm{~b}}$ K. Amako, ${ }^{65}$ P. Amaral,${ }^{29}$ C. Amelung, ${ }^{22}$ V. V. Ammosov, ${ }^{127}$ A. Amorim, ${ }^{123 a, c}$ G. Amorós, ${ }^{166}$ N. Amram, ${ }^{152}$ C. Anastopoulos, ${ }^{29}$ L. S. Ancu, ${ }^{16}$ N. Andari, ${ }^{114}$ T. Andeen, ${ }^{34}$ C. F. Anders, ${ }^{20}$ G. Anders,${ }^{57 a}$ K. J. Anderson, ${ }^{30}$ A. Andreazza, ${ }^{88 a, 88 b}$ V. Andrei, ${ }^{57 a}$ M-L. Andrieux, ${ }^{54}$ X. S. Anduaga, ${ }^{69}$ A. Angerami, ${ }^{34}$ F. Anghinolfi, ${ }^{29}$ A. Anisenkov, ${ }^{106}$ N. Anjos, ${ }^{123 a}$ A. Annovi, ${ }^{46}$ A. Antonaki, ${ }^{8}$ M. Antonelli, ${ }^{46}$ A. Antonov, ${ }^{95} \mathrm{~J}$. Antos, ${ }^{143 \mathrm{~b}}$ F. Anulli, ${ }^{131 \mathrm{a}} \mathrm{S}$. Aoun, ${ }^{82}$ L. Aperio Bella, ${ }^{4}$ R. Apolle, ${ }^{17, \mathrm{~d}} \mathrm{G}$. Arabidze, ${ }^{87}$ I. Aracena, ${ }^{142}$ Y. Arai, ${ }^{65}$ A. T. H. Arce, ${ }^{44}$ J. P. Archambault, ${ }^{28}$ S. Arfaoui, ${ }^{82}$ J-F. Arguin, ${ }^{14}$ E. Arik, ${ }^{18 a, a}$ M. Arik, ${ }^{18 a}$ A. J. Armbruster, ${ }^{86}$ O. Arnaez, ${ }^{80}$ A. Artamonov, ${ }^{94}$ G. Artoni, ${ }^{131 \mathrm{a}, 131 \mathrm{~b}}$ D. Arutinov, ${ }^{20} \mathrm{~S}$. Asai, ${ }^{154}$ R. Asfandiyarov,${ }^{171}$ S. Ask, ${ }^{27}$ B. Åsman, ${ }^{145 a, 145 b}$ L. Asquith, ${ }^{5}$ K. Assamagan, ${ }^{24}$ A. Astbury, ${ }^{168}$ A. Astvatsatourov, ${ }^{51}$ B. Aubert, ${ }^{4}$

E. Auge, ${ }^{114}$ K. Augsten, ${ }^{126}$ M. Aurousseau, ${ }^{144 a}$ G. Avolio, ${ }^{162}$ R. Avramidou, ${ }^{9}$ D. Axen,,${ }^{167}$ C. Ay, ${ }^{53}$ G. Azuelos, ${ }^{92, e}$ Y. Azuma, ${ }^{154}$ M. A. Baak, ${ }^{29}$ G. Baccaglioni, ${ }^{88 a}$ C. Bacci, ${ }^{133 a, 133 b}$ A. M. Bach, ${ }^{14}$ H. Bachacou, ${ }^{135}$ K. Bachas,${ }^{29}$ G. Bachy, ${ }^{29}$ M. Backes ${ }^{48}$ M. Backhaus,${ }^{20}$ E. Badescu, ${ }^{25 a}$ P. Bagnaia, ${ }^{131 a, 131 b}$ S. Bahinipati, ${ }^{2}$ Y. Bai, ${ }^{32 a}$ D. C. Bailey, ${ }_{157}^{17}$ T. Bain, ${ }^{157}$ J. T. Baines, ${ }^{128}$ O. K. Baker,${ }^{174}$ M. D. Baker, ${ }^{24}$ S. Baker, ${ }^{76}$ E. Banas, ${ }^{38}$ P. Banerjee, ${ }^{92}$ Sw. Banerjee, ${ }^{171}$ D. Banfi, ${ }^{29}$ A. Bangert, ${ }^{149}$ V. Bansal, ${ }^{168}$ H. S. Bansil, ${ }^{17}$ L. Barak, ${ }^{170}$ S. P. Baranov, ${ }^{93}$ A. Barashkou, ${ }^{64}$ A. Barbaro Galtieri, ${ }^{14}$ T. Barber, ${ }^{47}$ E. L. Barberio, ${ }^{85}$ D. Barberis, ${ }^{49 a, 49 b}$ M. Barbero, ${ }^{20}$ D. Y. Bardin, ${ }^{64}$ T. Barillari, ${ }^{98}$ M. Barisonzi, ${ }^{173}$ T. Barklow, ${ }^{142}$ N. Barlow, ${ }^{27}$ B. M. Barnett, ${ }^{128}$ R. M. Barnett, ${ }^{14}$ A. Baroncelli, ${ }^{133 a}$ G. Barone, ${ }^{48}$

A. J. Barr, ${ }^{117}$ F. Barreiro, ${ }^{79}$ J. Barreiro Guimarães da Costa ${ }^{56}$ R. Bartoldus, ${ }^{142}$ A. E. Barton, ${ }^{70}$ V. Bartsch, ${ }^{148}$ R. L. Bates, ${ }^{52}$ L. Batkova, ${ }^{143 a}$ J. R. Batley, ${ }^{27}$ A. Battaglia, ${ }^{16}$ M. Battistin, ${ }^{29}$ F. Bauer, ${ }^{135}$ H. S. Bawa, ${ }^{142, f}$ S. Beale, ${ }^{97}$ B. Beare, ${ }^{157}$ T. Beau, ${ }^{77}$ P. H. Beauchemin, ${ }^{160}$ R. Beccherle, ${ }^{49 a}$ P. Bechtle,${ }^{20}$ H. P. Beck, ${ }^{16}$ S. Becker, ${ }^{97}$ M. Beckingham, ${ }^{137}$ K. H. Becks, ${ }^{173}$ A. J. Beddall, ${ }^{18 \mathrm{c}}$ A. Beddall, ${ }^{18 \mathrm{c}}$ S. Bedikian, ${ }^{174}$ V. A. Bednyakov, ${ }^{64}$ C. P. Bee, ${ }^{82}$ M. Begel,${ }^{24}$ S. Behar Harpaz, ${ }^{151}$ P. K. Behera, ${ }^{62}$ M. Beimforde, ${ }^{98}$ C. Belanger-Champagne, ${ }^{84}$ P. J. Bell, ${ }^{48}$

W. H. Bell,${ }^{48}$ G. Bella, ${ }^{152}$ L. Bellagamba, ${ }^{19 a}$ F. Bellina, ${ }^{29}$ M. Bellomo, ${ }^{29}$ A. Belloni, ${ }^{56}$ O. Beloborodova, ${ }^{106}$ K. Belotskiy, ${ }^{95}$ O. Beltramello, ${ }^{29}$ S. Ben Ami, ${ }^{151}$ O. Benary,${ }^{152}$ D. Benchekroun, ${ }^{134 a}$ C. Benchouk, ${ }^{82}$ M. Bendel, ${ }^{80}$ N. Benekos, ${ }^{164}$ Y. Benhammou, ${ }^{152}$ E. Benhar Noccioli, ${ }^{48}$ J. A. Benitez Garcia, ${ }^{158 b}$ D. P. Benjamin, ${ }^{44}$ M. Benoit, ${ }^{114}$

J. R. Bensinger, ${ }^{22}$ K. Benslama, ${ }^{129}$ S. Bentvelsen, ${ }^{104}$ D. Berge, ${ }^{29}$ E. Bergeaas Kuutmann, ${ }^{41}$ N. Berger, ${ }^{4}$

F. Berghaus, ${ }^{168}$ E. Berglund, ${ }^{104}$ J. Beringer, ${ }^{14}$ P. Bernat,${ }^{76}$ R. Bernhard,${ }^{47}$ C. Bernius, ${ }^{24}$ T. Berry,${ }^{75}$ C. Bertella, ${ }^{82}$ A. Bertin, ${ }^{19 a, 19 b}$ F. Bertinelli, ${ }^{29}$ F. Bertolucci, ${ }^{121 \mathrm{a}, 121 \mathrm{~b}}$ M. I. Besana ${ }^{88 \mathrm{a}, 88 \mathrm{~b}}$ N. Besson, ${ }^{135}$ S. Bethke, ${ }^{98} \mathrm{~W}$. Bhimji ${ }^{45}$ R. M. Bianchi, ${ }^{29}$ M. Bianco, ${ }^{71 \mathrm{a}, 71 \mathrm{~b}}$ O. Biebel,${ }^{97}$ S. P. Bieniek, ${ }^{76}$ K. Bierwagen, ${ }^{53}$ J. Biesiada, ${ }^{14}$ M. Biglietti, ${ }^{133 a, 133 b}$ 
H. Bilokon, ${ }^{46}$ M. Bindi, ${ }^{19 a, 19 b}$ S. Binet, ${ }^{114}$ A. Bingul, ${ }^{18 c}$ C. Bini,,${ }^{131 a, 131 b}$ C. Biscarat, ${ }^{176}$ U. Bitenc,${ }^{47}$ K. M. Black, ${ }^{21}$ R. E. Blair, ${ }^{5}$ J.-B. Blanchard, ${ }^{114}$ G. Blanchot, ${ }^{29}$ T. Blazek, ${ }^{143 a}$ C. Blocker, ${ }^{22}$ J. Blocki, ${ }^{38}$ A. Blondel,${ }^{48}$ W. Blum, ${ }^{80}$ U. Blumenschein, ${ }^{53}$ G. J. Bobbink, ${ }^{104}$ V. B. Bobrovnikov, ${ }^{106}$ S. S. Bocchetta, ${ }^{78}$ A. Bocci, ${ }^{44}$ C. R. Boddy,,${ }^{117}$ M. Boehler, ${ }^{41}$ J. Boek, ${ }^{173}$ N. Boelaert, ${ }^{35}$ S. Böser, ${ }^{76}$ J. A. Bogaerts, ${ }^{29}$ A. Bogdanchikov, ${ }^{106}$ A. Bogouch,${ }^{89, a}$ C. Bohm, ${ }^{145 a}$ V. Boisvert, ${ }^{75}$ T. Bold,${ }^{37}$ V. Boldea, ${ }^{25 a}$ N. M. Bolnet, ${ }^{135}$ M. Bona ${ }^{74}$ V. G. Bondarenko, ${ }^{95}$ M. Bondioli, ${ }^{162}$ M. Boonekamp, ${ }^{135}$ G. Boorman, ${ }^{75}$ C. N. Booth, ${ }^{138}$ S. Bordoni, ${ }^{77}$ C. Borer, ${ }^{16}$ A. Borisov, ${ }^{127}$ G. Borissov, ${ }^{70}$ I. Borjanovic, ${ }^{12 a}$ S. Borroni, ${ }^{86}$ K. Bos, ${ }^{104}$ D. Boscherini, ${ }^{19 a}$ M. Bosman, ${ }^{11}$ H. Boterenbrood,${ }^{104}$ D. Botterill, ${ }^{128}$ J. Bouchami, ${ }^{92}$ J. Boudreau, ${ }^{122}$ E. V. Bouhova-Thacker, ${ }^{70}$ D. Boumediene, ${ }^{33}$ C. Bourdarios,${ }^{114}$ N. Bousson, ${ }^{82}$ A. Boveia,${ }^{30}$ J. Boyd ${ }^{29}$ I. R. Boyko, ${ }^{64}$ N. I. Bozhko, ${ }^{127}$ I. Bozovic-Jelisavcic, ${ }^{12 b}$ J. Bracinik, ${ }^{17}$ A. Braem, ${ }^{29}$ P. Branchini, ${ }^{133 a}$ G. W. Brandenburg, ${ }^{56}$ A. Brandt, ${ }^{7}$ G. Brandt, ${ }^{117}$ O. Brandt ${ }^{53}$ U. Bratzler, ${ }^{155}$ B. Brau, ${ }^{83}$ J. E. Brau, ${ }^{113}$ H. M. Braun, ${ }^{173}$ B. Brelier, ${ }^{157}$ J. Bremer, ${ }^{29}$ R. Brenner, ${ }^{165}$ S. Bressler, ${ }^{170}$ D. Breton, ${ }^{114}$ D. Britton, ${ }^{52}$ F. M. Brochu, ${ }^{27}$ I. Brock,${ }^{20}$ R. Brock,${ }^{87}$ T. J. Brodbeck, ${ }^{70}$ E. Brodet,${ }^{152}$ F. Broggi, ${ }^{88 a}$ C. Bromberg, ${ }^{87}$ J. Bronner,${ }^{98}$ G. Brooijmans, ${ }^{34}$ W. K. Brooks, ${ }^{31 b}$ G. Brown, ${ }^{81}$ H. Brown, ${ }^{7}$ P. A. Bruckman de Renstrom, ${ }^{38}$ D. Bruncko, ${ }^{143 b}$

R. Bruneliere, ${ }^{47}$ S. Brunet ${ }^{60}$ A. Bruni, ${ }^{19 a}$ G. Bruni, ${ }^{19 a}$ M. Bruschi, ${ }^{19 a}$ T. Buanes, ${ }^{13}$ Q. Buat, ${ }^{54}$ F. Bucci ${ }^{48}$ J. Buchanan, ${ }^{117}$ N. J. Buchanan, ${ }^{2}$ P. Buchholz, ${ }^{140}$ R. M. Buckingham, ${ }^{117}$ A. G. Buckley, ${ }^{45}$ S. I. Buda, ${ }^{25 a}$ I. A. Budagov, ${ }^{64}$ B. Budick, ${ }^{107}$ V. Büscher ${ }^{80}$ L. Bugge, ${ }^{116}$ O. Bulekov, ${ }^{95}$ M. Bunse, ${ }^{42}$ T. Buran,,${ }^{16}$ H. Burckhart,${ }^{29}$ S. Burdin,${ }^{72}$ T. Burgess,${ }^{13}$ S. Burke, ${ }^{128}$ E. Busato, ${ }^{33}$ P. Bussey,${ }^{52}$ C. P. Buszello,,${ }^{165}$ F. Butin, ${ }^{29}$ B. Butler,${ }^{142}$ J. M. Butler, ${ }^{21}$ C. M. Buttar, ${ }^{52}$ J. M. Butterworth, ${ }^{76}$ W. Buttinger, ${ }^{27}$ S. Cabrera Urbán, ${ }^{166}$ D. Caforio, ${ }^{19 a, 19 b}$ O. Cakir, ${ }^{3 a}$ P. Calafiura, ${ }^{14}$ G. Calderini,${ }^{77}$ P. Calfayan, ${ }^{97}$ R. Calkins, ${ }^{105}$ L. P. Caloba,${ }^{23 a}$ R. Caloi, ${ }^{131 a, 131 b}$ D. Calvet, ${ }^{33}$ S. Calvet, ${ }^{33}$ R. Camacho Toro, ${ }^{33}$ P. Camarri, ${ }^{132 a, 1323 b}$ M. Cambiaghi, ${ }^{118 a, 118 b}$ D. Cameron, ${ }^{116}$ L. M. Caminada, ${ }^{14}$ S. Campana, ${ }^{29}$ M. Campanelli, ${ }^{76}$ V. Canale,${ }^{101 \mathrm{a}, 101 \mathrm{~b}}$ F. Canelli, ${ }^{30, \mathrm{~g}}$ A. Canepa, ${ }^{158 \mathrm{a}}$ J. Cantero, ${ }^{79}$ L. Capasso, ${ }^{101 \mathrm{a}, 101 \mathrm{~b}}$ M. D. M. Capeans Garrido, ${ }^{29}$ I. Caprini, ${ }^{25 a}$ M. Caprini, ${ }^{25 a}$ D. Capriotti, ${ }^{98}$ M. Capua,${ }^{36 a, 36 b}$ R. Caputo,${ }^{80}$ R. Cardarelli, ${ }^{132 \mathrm{a}}$ T. Carli, ${ }^{29}$ G. Carlino, ${ }^{101 \mathrm{a}}$ L. Carminati, ${ }^{88 a, 88 \mathrm{~b}}$ S. Caron,${ }^{47}$ G. D. Carrillo Montoya, ${ }^{171}$ A. A. Carter ${ }^{74}$ J. R. Carter, ${ }^{27}$ J. Carvalho, ${ }^{123 a, h}$ D. Casadei, ${ }^{107}$ M. P. Casado, ${ }^{11}$ M. Cascella, ${ }^{121 a, 121 b}$ C. Caso, ${ }^{49 a, 49 b, a}$ A. M. Castaneda Hernandez, ${ }^{171}$ E. Castaneda-Miranda, ${ }^{171}$ V. Castillo Gimenez, ${ }^{166}$ N. F. Castro, ${ }^{123 a}$ G. Cataldi, ${ }^{71 a}$ F. Cataneo, ${ }^{29}$ A. Catinaccio, ${ }^{29}$ J. R. Catmore, ${ }^{70}$ A. Cattai, ${ }^{29}$ G. Cattani, ${ }^{132 a, 1323 b}$ S. Caughron, ${ }^{87}$ D. Cauz, ${ }^{163 a, 163 c}$ P. Cavalleri, ${ }^{77}$ D. Cavalli, ${ }^{88 a}$ M. Cavalli-Sforza,${ }^{11}$ V. Cavasinni, ${ }^{121 a, 121 b}$ F. Ceradini, ${ }^{133 a, 133 b}$ A. S. Cerqueira, ${ }^{23 b}$ A. Cerri, ${ }^{29}$ L. Cerrito,${ }^{74}$ F. Cerutti, ${ }^{46}$ S. A. Cetin, ${ }^{18 b}$ F. Cevenini, ${ }^{101 a, 101 b}$ A. Chafaq, ${ }^{134 a}$ D. Chakraborty, ${ }^{105}$ K. Chan, ${ }^{2}$ B. Chapleau, ${ }^{84}$ J. D. Chapman, ${ }^{27}$ J. W. Chapman, ${ }^{86}$ E. Chareyre, ${ }^{77}$ D. G. Charlton, ${ }^{17}$ V. Chavda,${ }^{81}$

C. A. Chavez Barajas, ${ }^{29}$ S. Cheatham,${ }^{84}$ S. Chekanov, ${ }^{5}$ S. V. Chekulaev, ${ }^{158 a}$ G. A. Chelkov, ${ }^{64}$ M. A. Chelstowska, ${ }^{103}$ C. Chen,${ }^{63}$ H. Chen, ${ }^{24}$ S. Chen, ${ }^{32 \mathrm{c}}$ T. Chen,${ }^{32 \mathrm{c}}$ X. Chen, ${ }^{171}$ S. Cheng, ${ }^{32 \mathrm{a}}$ A. Cheplakov, ${ }^{64}$ V. F. Chepurnov, ${ }^{64}$ R. Cherkaoui El Moursli, ${ }^{134 \mathrm{e}}$ V. Chernyatin, ${ }^{24}$ E. Cheu, ${ }^{6}$ S. L. Cheung, ${ }^{157}$ L. Chevalier, ${ }^{135}$ G. Chiefari, ${ }^{101 a, 101 b}$ L. Chikovani, ${ }^{50 a}$ J. T. Childers, ${ }^{57 a}$ A. Chilingarov, ${ }^{70}$ G. Chiodini, ${ }^{71 a}$ M. V. Chizhov ${ }^{64}$ G. Choudalakis,${ }^{30}$ S. Chouridou, ${ }^{136}$ I. A. Christidi, ${ }^{76}$ A. Christov, ${ }^{47}$ D. Chromek-Burckhart, ${ }^{29}$ M. L. Chu, ${ }^{150}$ J. Chudoba, ${ }^{124}$ G. Ciapetti, ${ }^{131 \mathrm{a}, 13 \mathrm{bb}}$ K. Ciba, ${ }^{37}$ A. K. Ciftci, ${ }^{3 \mathrm{a}}$ R. Ciftci, ${ }^{3 \mathrm{a}}$ D. Cinca,${ }^{33}$ V. Cindro, ${ }^{73}$ M. D. Ciobotaru, ${ }^{162}$ C. Ciocca, ${ }^{19 a}$ A. Ciocio, ${ }^{14}$ M. Cirilli, ${ }^{86}$ M. Ciubancan, ${ }^{25 a}$ A. Clark, ${ }^{48}$ P. J. Clark,${ }^{45}$ W. Cleland, ${ }^{122}$ J. C. Clemens, ${ }^{82}$ B. Clement, ${ }^{54}$ C. Clement, ${ }^{145 a, 145 b}$ R. W. Clifft, ${ }^{128}$ Y. Coadou, ${ }^{82}$ M. Cobal, ${ }^{163 a, 163 c}$ A. Coccaro, ${ }^{49 a, 49 b}$ J. Cochran, ${ }^{63}$ P. Coe ${ }^{117}$ J. G. Cogan, ${ }^{142}$ J. Coggeshall, ${ }^{164}$ E. Cogneras, ${ }^{176}$ J. Colas,${ }^{4}$ A. P. Colijn, ${ }^{104}$ C. Collard, ${ }^{114}$ N. J. Collins, ${ }^{17}$

C. Collins-Tooth, ${ }^{52}$ J. Collot, ${ }^{54}$ G. Colon, ${ }^{83}$ P. Conde Muiño, ${ }^{123 a}$ E. Coniavitis, ${ }^{117}$ M. C. Conidi, ${ }^{11}$ M. Consonni, ${ }^{103}$ V. Consorti, ${ }^{47}$ S. Constantinescu, ${ }^{25 a}$ C. Conta, ${ }^{118 a, 118 b}$ F. Conventi, ${ }^{101 a, i}$ J. Cook,${ }^{29}$ M. Cooke, ${ }^{14}$ B. D. Cooper, ${ }^{76}$ A. M. Cooper-Sarkar, ${ }^{117}$ K. Copic, ${ }^{14}$ T. Cornelissen, ${ }^{173}$ M. Corradi, ${ }^{19 a}$ F. Corriveau, ${ }^{84, j}$ A. Cortes-Gonzalez, ${ }^{164}$ G. Cortiana, ${ }^{98}$ G. Costa, ${ }^{88 a}$ M. J. Costa, ${ }^{166}$ D. Costanzo, ${ }^{138}$ T. Costin,${ }^{30}$ D. Côté, ${ }^{29}$ L. Courneyea,${ }^{168}$ G. Cowan, ${ }^{75}$ C. Cowden,${ }^{27}$ B. E. Cox,${ }^{81}$ K. Cranmer, ${ }^{107}$ F. Crescioli, ${ }^{121 a, 121 b}$ M. Cristinziani, ${ }^{20}$ G. Crosetti, ${ }^{36 a, 36 b}$ R. Crupi, ${ }^{71 a, 71 b}$ S. Crépé-Renaudin, ${ }^{54}$ C.-M. Cuciuc, ${ }^{25 a}$ C. Cuenca Almenar, ${ }^{174}$ T. Cuhadar Donszelmann, ${ }^{138}$ M. Curatolo, ${ }^{46}$ C. J. Curtis, ${ }^{17}$ C. Cuthbert, ${ }^{149}$ P. Cwetanski, ${ }^{60}$ H. Czirr, ${ }^{140}$ P. Czodrowski, ${ }^{43}$ Z. Czyczula, ${ }^{174}$ S. D'Auria, ${ }^{52}$ M. D’Onofrio, ${ }^{72}$ A. D’ Orazio, ${ }^{131 a, 131 b}$ P. V. M. Da Silva, ${ }^{23 a}$ C. Da Via, ${ }^{81}$ W. Dabrowski, ${ }^{37}$ T. Dai, ${ }^{86}$ C. Dallapiccola, ${ }^{83}$

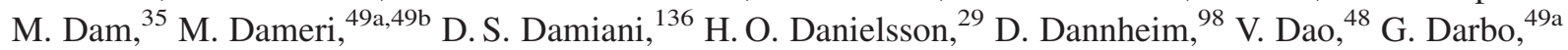
G. L. Darlea, ${ }^{25 b}$ C. Daum, ${ }^{104}$ W. Davey,${ }^{20}$ T. Davidek, ${ }^{125}$ N. Davidson,,${ }^{85}$ R. Davidson,${ }^{70}$ E. Davies, ${ }^{117, d}$ M. Davies, ${ }^{92}$ A. R. Davison, ${ }^{76}$ Y. Davygora, ${ }^{57 a}$ E. Dawe, ${ }^{141}$ I. Dawson, ${ }^{138}$ J. W. Dawson,,${ }^{5, a}$ R. K. Daya, ${ }^{22}$ K. De, ${ }^{7}$

R. de Asmundis, ${ }^{101 \mathrm{a}}$ S. De Castro, ${ }^{19 a, 19 \mathrm{~b}}$ P. E. De Castro Faria Salgado, ${ }^{24}$ S. De Cecco,${ }^{77}$ J. de Graat,${ }^{97}$ N. De Groot, ${ }^{103}$ P. de Jong, ${ }^{104}$ C. De La Taille, ${ }^{114}$ H. De la Torre, ${ }^{79}$ B. De Lotto, ${ }^{163 a, 163 c}$ L. de Mora, ${ }^{70}$ L. De Nooij, ${ }^{104}$ 
D. De Pedis, ${ }^{131 a}$ A. De Salvo, ${ }^{131 a}$ U. De Sanctis, ${ }^{163 a, 163 c}$ A. De Santo, ${ }^{148}$ J. B. De Vivie De Regie, ${ }^{114}$ S. Dean, ${ }^{76}$ W. J. Dearnaley, ${ }^{70}$ R. Debbe,${ }^{24}$ C. Debenedetti, ${ }^{45}$ D. V. Dedovich,${ }^{64}$ J. Degenhardt, ${ }^{119}$ M. Dehchar, ${ }^{117}$ C. Del Papa ${ }^{163 a, 163 c}$ J. Del Peso,${ }^{79}$ T. Del Prete, ${ }^{121 a, 121 b}$ T. Delemontex,${ }^{54}$ M. Deliyergiyev, ${ }^{73}$ A. Dell' Acqua ${ }^{29}$ L. Dell'Asta, ${ }^{21}$ M. Della Pietra, ${ }^{101 a, i}$ D. della Volpe,${ }^{101 a, 101 b}$ M. Delmastro, ${ }^{4}$ N. Delruelle,${ }^{29}$ P. A. Delsart,${ }^{54}$ C. Deluca, ${ }^{147}$ S. Demers, ${ }^{174}$ M. Demichev, ${ }^{64}$ B. Demirkoz,${ }^{11, k}$ J. Deng, ${ }^{162}$ S. P. Denisov, ${ }^{127}$ D. Derendarz,${ }^{38}$ J. E. Derkaoui, ${ }^{134 d}$ F. Derue,${ }^{77}$ P. Dervan, ${ }^{72}$ K. Desch,${ }^{20}$ E. Devetak,${ }^{147}$ P. O. Deviveiros, ${ }^{104}$ A. Dewhurst,${ }^{128}$ B. DeWilde, ${ }^{147}$ S. Dhaliwal, ${ }^{157}$ R. Dhullipudi, ${ }^{24,1}$ A. Di Ciaccio, ${ }^{132 a, 1323 b}$ L. Di Ciaccio, ${ }^{4}$ A. Di Girolamo, ${ }^{29}$ B. Di Girolamo, ${ }^{29}$ S. Di Luise, ${ }^{133 a, 133 b}$ A. Di Mattia, ${ }^{171}$ B. Di Micco,${ }^{29}$ R. Di Nardo, ${ }^{46}$ A. Di Simone, ${ }^{132 a, 1323 b}$ R. Di Sipio, ${ }^{19 a, 19 b}$ M. A. Diaz, ${ }^{31 a}$ F. Diblen, ${ }^{18 c}$ E. B. Diehl, ${ }^{86}$ J. Dietrich, ${ }^{41}$ T. A. Dietzsch, ${ }^{57 a}$ S. Diglio, ${ }^{85}$ K. Dindar Yagci, ${ }^{39}$ J. Dingfelder, ${ }^{20}$ C. Dionisi, ${ }^{131 a, 131 b}$ P. Dita, ${ }^{25 a}$ S. Dita, ${ }^{25 a}$ F. Dittus, ${ }^{29}$ F. Djama, ${ }^{82}$ T. Djobava, ${ }^{50 b}$

M. A. B. do Vale, ${ }^{23 c}$ A. Do Valle Wemans, ${ }^{123 a}$ T. K. O. Doan, ${ }^{4}$ M. Dobbs,${ }^{84}$ R. Dobinson, ${ }^{29, a}$ D. Dobos, ${ }^{29}$ E. Dobson,${ }^{29, \mathrm{~m}}$ M. Dobson, ${ }^{162}$ J. Dodd ${ }^{34}$ C. Doglioni, ${ }^{117}$ T. Doherty ${ }^{52}$ Y. Doi,${ }^{65, a}$ J. Dolejsi, ${ }^{125}$ I. Dolenc, ${ }^{73}$ Z. Dolezal, ${ }^{125}$ B. A. Dolgoshein, ${ }^{95, a}$ T. Dohmae, ${ }^{154}$ M. Donadelli, ${ }^{23 d}$ M. Donega, ${ }^{119}$ J. Donini, ${ }^{33}$ J. Dopke, ${ }^{29}$ A. Doria, ${ }^{101 \mathrm{a}}$ A. Dos Anjos, ${ }^{171}$ M. Dosil,${ }^{11}$ A. Dotti, ${ }^{121 \mathrm{a}, 121 \mathrm{~b}}$ M. T. Dova, ${ }^{69}$ J. D. Dowell, ${ }^{17}$ A. D. Doxiadis, ${ }^{104}$ A. T. Doyle, ${ }^{52}$ Z. Drasal, ${ }^{125}$ J. Drees,${ }^{173}$ N. Dressnandt, ${ }^{119}$ H. Drevermann, ${ }^{29}$ C. Driouichi, ${ }^{35}$ M. Dris, ${ }^{9}$ J. Dubbert,${ }^{98}$ S. Dube, ${ }^{14}$ E. Duchovni, ${ }^{170}$ G. Duckeck, ${ }^{97}$ A. Dudarev, ${ }^{29}$ F. Dudziak,${ }^{63}$ M. Dührssen, ${ }^{29}$ I. P. Duerdoth, ${ }^{81}$ L. Duflot,,${ }^{114}$

M-A. Dufour, ${ }^{84}$ M. Dunford, ${ }^{29}$ H. Duran Yildiz,${ }^{3 b}$ R. Duxfield, ${ }^{138}$ M. Dwuznik, ${ }^{37}$ F. Dydak, ${ }^{29}$ M. Düren, ${ }^{51}$

W. L. Ebenstein,${ }^{44}$ J. Ebke, ${ }^{97}$ S. Eckweiler, ${ }^{80}$ K. Edmonds, ${ }^{80}$ C. A. Edwards,${ }^{75}$ N. C. Edwards,${ }^{52}$ W. Ehrenfeld, ${ }^{41}$ T. Ehrich, ${ }^{98}$ T. Eifert, ${ }^{29}$ G. Eigen,${ }^{13}$ K. Einsweiler, ${ }^{14}$ E. Eisenhandler, ${ }^{74}$ T. Ekelof, ${ }^{165}$ M. El Kacimi, ${ }^{134 c}$ M. Ellert, ${ }^{165}$ S. Elles, ${ }^{4}$ F. Ellinghaus, ${ }^{80}$ K. Ellis,${ }^{74}$ N. Ellis,${ }^{29}$ J. Elmsheuser, ${ }^{97}$ M. Elsing, ${ }^{29}$ D. Emeliyanov, ${ }^{128}$ R. Engelmann, ${ }^{147}$ A. Engl, ${ }^{97}$ B. Epp ${ }^{61}$ A. Eppig, ${ }^{86}$ J. Erdmann, ${ }^{53}$ A. Ereditato, ${ }^{16}$ D. Eriksson, ${ }^{145 a}$ J. Ernst, ${ }^{1}$ M. Ernst, ${ }^{24}$ J. Ernwein, ${ }^{135}$ D. Errede,${ }^{164}$ S. Errede, ${ }^{164}$ E. Ertel,${ }^{80}$ M. Escalier, ${ }^{114}$ C. Escobar, ${ }^{122}$ X. Espinal Curull, ${ }^{11}$ B. Esposito, ${ }^{46}$ F. Etienne, ${ }^{82}$

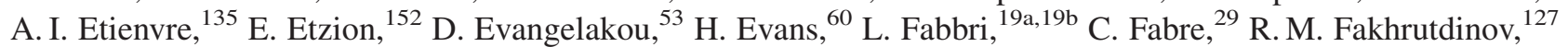
S. Falciano, ${ }^{131 \mathrm{a}}$ Y. Fang, ${ }^{171}$ M. Fanti, ${ }^{88 a}, 88 \mathrm{~b}$ A. Farbin, ${ }^{7}$ A. Farilla, ${ }^{133 \mathrm{a}}$ J. Farley, ${ }^{147}$ T. Farooque, ${ }^{157}$

S. M. Farrington, ${ }^{117}$ P. Farthouat, ${ }^{29}$ P. Fassnacht, ${ }^{29}$ D. Fassouliotis,${ }^{8}$ B. Fatholahzadeh, ${ }^{157}$ A. Favareto, ${ }^{88 a, 88 b}$ L. Fayard, ${ }^{114}$ S. Fazio, ${ }^{36 a, 36 b}$ R. Febbraro, ${ }^{33}$ P. Federic, ${ }^{143 a}$ O. L. Fedin, ${ }^{120}$ W. Fedorko,${ }^{87}$ M. Fehling-Kaschek, ${ }^{47}$ L. Feligioni, ${ }^{82}$ C. Feng, ${ }^{32 d}$ E. J. Feng, ${ }^{30}$ A. B. Fenyuk, ${ }^{127}$ J. Ferencei, ${ }^{143 b}$ J. Ferland,${ }^{92}$ W. Fernando, ${ }^{108}$ S. Ferrag,${ }^{52}$ J. Ferrando, ${ }^{52}$ V. Ferrara, ${ }^{41}$ A. Ferrari, ${ }^{165}$ P. Ferrari, ${ }^{104}$ R. Ferrari, ${ }^{118 a}$ A. Ferrer, ${ }^{166}$ M. L. Ferrer, ${ }^{46}$ D. Ferrere, ${ }^{48}$ C. Ferretti, ${ }^{86}$ A. Ferretto Parodi, ${ }^{49 a, 49 b}$ M. Fiascaris,${ }^{30}$ F. Fiedler, ${ }^{80}$ A. Filipčič, ${ }^{73}$ A. Filippas, ${ }^{9}$ F. Filthaut, ${ }^{103}$ M. Fincke-Keeler, ${ }^{168}$ M. C. N. Fiolhais, ${ }^{123 a, h}$ L. Fiorini,${ }^{166}$ A. Firan, ${ }^{39}$ P. Fischer,${ }^{20}$ M. J. Fisher,${ }^{108}$ M. Flechl, ${ }^{47}$ I. Fleck, ${ }^{140}$ J. Fleckner ${ }^{80}$ P. Fleischmann, ${ }^{172}$ S. Fleischmann, ${ }^{173}$ T. Flick, ${ }^{173}$ L. R. Flores Castillo, ${ }^{171}$ M. J. Flowerdew,${ }^{98}$ M. Fokitis, ${ }^{9}$ T. Fonseca Martin, ${ }^{16}$ D. A. Forbush, ${ }^{137}$ A. Formica, ${ }^{135}$ A. Forti ${ }^{81}$ D. Fortin, ${ }^{158 a}$ J. M. Foster, ${ }^{81}$ D. Fournier, ${ }^{114}$ A. Foussat, ${ }^{29}$ A. J. Fowler, ${ }^{44}$ K. Fowler, ${ }^{136}$ H. Fox, ${ }^{70}$ P. Francavilla, ${ }^{121 a, 121 b}$ S. Franchino, ${ }^{118 \mathrm{a}, 118 \mathrm{~b}}$ D. Francis, ${ }^{29}$ T. Frank, ${ }^{170}$ M. Franklin, ${ }^{56}$ S. Franz,${ }^{29}$ M. Fraternali, ${ }^{118 \mathrm{a}, 118 \mathrm{~b}}$ S. Fratina, ${ }^{119}$ S. T. French, ${ }^{27}$ F. Friedrich, ${ }^{43}$ R. Froeschl,${ }^{29}$ D. Froidevaux, ${ }^{29}$ J. A. Frost, ${ }^{27}$ C. Fukunaga, ${ }^{155}$ E. Fullana Torregrosa, ${ }^{29}$ J. Fuster, ${ }^{166}$ C. Gabaldon, ${ }^{29}$ O. Gabizon, ${ }^{170}$ T. Gadfort ${ }^{24}$ S. Gadomski, ${ }^{48}$ G. Gagliardi, ${ }^{49,49 b}$ P. Gagnon, ${ }^{60}$ C. Galea, ${ }^{97}$ E. J. Gallas, ${ }^{117}$ V. Gallo, ${ }^{16}$ B. J. Gallop, ${ }^{128}$ P. Gallus, ${ }^{124}$ K. K. Gan, ${ }^{108}$ Y. S. Gao, ${ }^{142, f}$ V. A. Gapienko, ${ }^{127}$ A. Gaponenko, ${ }^{14}$ F. Garberson, ${ }^{174}$ M. Garcia-Sciveres, ${ }^{14}$ C. García, ${ }^{166}$ J. E. García Navarro, ${ }^{166}$ R. W. Gardner, ${ }^{30}$ N. Garelli, ${ }^{29}$ H. Garitaonandia, ${ }^{104}$ V. Garonne, ${ }^{29}$ J. Garvey, ${ }^{17}$ C. Gatti, ${ }^{46}$ G. Gaudio, ${ }^{118 a}$ O. Gaumer, ${ }^{48}$ B. Gaur, ${ }^{140}$ L. Gauthier, ${ }^{135}$ I. L. Gavrilenko, ${ }^{93}$ C. Gay,${ }^{167}$ G. Gaycken, ${ }^{20}$ J-C. Gayde,${ }^{29}$ E. N. Gazis, ${ }^{9}$ P. Ge,${ }^{32 d}$ C. N. P. Gee, ${ }^{128}$ D. A. A. Geerts, ${ }^{104}$ Ch. Geich-Gimbel, ${ }^{20}$ K. Gellerstedt, ${ }^{145 a, 145 b}$ C. Gemme ${ }^{49 a}$ A. Gemmell, ${ }^{52}$ M. H. Genest,${ }^{97}$ S. Gentile, ${ }^{131 \mathrm{a}, 131 \mathrm{~b}}$ M. George ${ }^{53}$ S. George,${ }^{75}$ P. Gerlach, ${ }^{173}$ A. Gershon, ${ }^{152}$ C. Geweniger,${ }^{57 a}$ H. Ghazlane, ${ }^{134 b}$ N. Ghodbane, ${ }^{33}$ B. Giacobbe, ${ }^{19 a}$ S. Giagu, ${ }^{131 a, 131 b}$ V. Giakoumopoulou, ${ }^{8}$ F. Gianotti, ${ }^{29}$ B. Gibbard ${ }^{24}$ A. Gibson, ${ }^{157}$ S. M. Gibson, ${ }^{29}$ L. M. Gilbert, ${ }^{117}$ V. Gilewsky, ${ }^{90}$ D. Gillberg, ${ }^{28}$ A. R. Gillman, ${ }^{128}$ D. M. Gingrich,${ }^{2, e}$ J. Ginzburg, ${ }^{152}$ N. Giokaris, ${ }^{8}$ M. P. Giordani, ${ }^{163 \mathrm{c}}$ R. Giordano, ${ }^{101 \mathrm{a}, 101 \mathrm{~b}}$ F. M. Giorgi, ${ }^{15}$ P. Giovannini, ${ }^{98}$ P. F. Giraud, ${ }^{135}$ D. Giugni, ${ }^{88 \mathrm{a}}$ M. Giunta, ${ }^{92}$ P. Giusti, ${ }^{19 a}$ B. K. Gjelsten, ${ }^{116}$ L. K. Gladilin, ${ }^{96}$ C. Glasman, ${ }^{79}$ J. Glatzer, ${ }^{47}$ A. Glazov, ${ }^{41}$ G. L. Glonti, ${ }^{64}$ J. R. Goddard, ${ }^{74}$ J. Godfrey, ${ }^{141}$ J. Godlewski, ${ }^{29}$ M. Goebel, ${ }^{41}$ T. Göpfert ${ }^{43}$ C. Goeringer, ${ }^{80}$ C. Gössling, ${ }^{42}$

T. Göttfert, ${ }^{98}$ S. Goldfarb, ${ }^{86}$ T. Golling, ${ }^{174}$ S. N. Golovnia, ${ }^{127}$ A. Gomes, ${ }^{123 a, c}$ L. S. Gomez Fajardo, ${ }^{41}$ R. Gonçalo, ${ }^{75}$ J. Goncalves Pinto Firmino Da Costa, ${ }^{41}$ L. Gonella, ${ }^{20}$ A. Gonidec, ${ }^{29}$ S. Gonzalez, ${ }^{171}$ S. González de la Hoz, ${ }^{166}$ G. Gonzalez Parra, ${ }^{11}$ M. L. Gonzalez Silva, ${ }^{26}$ S. Gonzalez-Sevilla, ${ }^{48}$ J. J. Goodson, ${ }^{147}$ L. Goossens, ${ }^{29}$ P. A. Gorbounov, ${ }^{94}$ H. A. Gordon, ${ }^{24}$ I. Gorelov, ${ }^{102}$ G. Gorfine, ${ }^{173}$ B. Gorini, ${ }^{29}$ E. Gorini, ${ }^{71 a, 71 b}$ A. Gorišek, ${ }^{73}$ 
E. Gornicki, ${ }^{38}$ S. A. Gorokhov, ${ }^{127}$ V. N. Goryachev, ${ }^{127}$ B. Gosdzik, ${ }^{41}$ M. Gosselink, ${ }^{104}$ M. I. Gostkin, ${ }^{64}$ I. Gough Eschrich, ${ }^{162}$ M. Gouighri, ${ }^{134 a}$ D. Goujdami, ${ }^{134 \mathrm{c}}$ M. P. Goulette, ${ }^{48}$ A. G. Goussiou, ${ }^{137}$ C. Goy, ${ }^{4}$ S. Gozpinar, ${ }^{22}$ I. Grabowska-Bold, ${ }^{37}$ P. Grafström, ${ }^{29}$ K-J. Grahn, ${ }^{41}$ F. Grancagnolo, ${ }^{71 a}$ S. Grancagnolo, ${ }^{15}$ V. Grassi, ${ }^{147}$ V. Gratchev, ${ }^{120}$ N. Grau, ${ }^{34}$ H. M. Gray, ${ }^{29}$ J. A. Gray,${ }^{147}$ E. Graziani, ${ }^{133 a}$ O. G. Grebenyuk, ${ }^{120}$ T. Greenshaw, ${ }^{72}$ Z. D. Greenwood,${ }^{24,1}$ K. Gregersen,${ }^{35}$ I. M. Gregor, ${ }^{41}$ P. Grenier, ${ }^{142}$ J. Griffiths, ${ }^{137}$ N. Grigalashvili, ${ }^{64}$ A. A. Grillo, ${ }^{136}$ S. Grinstein, ${ }^{11}$ Y. V. Grishkevich, ${ }^{96}$ J.-F. Grivaz, ${ }^{114}$ M. Groh, ${ }^{98}$ E. Gross, ${ }^{170}$ J. Grosse-Knetter ${ }^{53}$ J. Groth-Jensen, ${ }^{170}$ K. Grybel, ${ }^{140}$ V. J. Guarino, ${ }^{5}$ D. Guest, ${ }^{174}$ C. Guicheney, ${ }^{33}$ A. Guida, ${ }^{71 a, 71 b}$ S. Guindon, ${ }^{53}$ H. Guler ${ }^{84, n}$ J. Gunther, ${ }^{124}$ B. Guo, ${ }^{157}$ J. Guo, ${ }^{34}$ A. Gupta,${ }^{30}$ Y. Gusakov, ${ }^{64}$ V. N. Gushchin, ${ }^{127}$ A. Gutierrez, ${ }^{92}$ P. Gutierrez, ${ }^{110}$ N. Guttman, ${ }^{152}$ O. Gutzwiller, ${ }^{171}$ C. Guyot, ${ }^{135}$ C. Gwenlan, ${ }^{117}$ C. B. Gwilliam, ${ }^{72}$ A. Haas, ${ }^{142}$ S. Haas,${ }^{29}$ C. Haber, ${ }^{14}$ R. Hackenburg, ${ }^{24}$ H. K. Hadavand, ${ }^{39}$ D. R. Hadley,${ }^{17}$ P. Haefner, ${ }^{98}$ F. Hahn, ${ }^{29}$ S. Haider, ${ }^{29}$ Z. Hajduk,${ }^{38}$ H. Hakobyan,,${ }^{175}$ J. Haller, ${ }^{53}$ K. Hamacher, ${ }^{173}$ P. Hamal, ${ }^{112}$ M. Hamer, ${ }^{53}$ A. Hamilton, ${ }^{144 b}$ S. Hamilton, ${ }^{160}$ H. Han,${ }^{32 a}$ L. Han,${ }^{32 b}$ K. Hanagaki, ${ }^{115}$ K. Hanawa, ${ }^{159}$ M. Hance,${ }^{14}$ C. Handel,${ }^{80}$ P. Hanke,${ }^{57 a}$ J. R. Hansen, ${ }^{35}$ J. B. Hansen, ${ }^{35}$ J. D. Hansen, ${ }^{35}$ P. H. Hansen, ${ }^{35}$ P. Hansson, ${ }^{142}$ K. Hara, ${ }^{159}$ G. A. Hare, ${ }^{136}$ T. Harenberg, ${ }^{173}$ S. Harkusha, ${ }^{89}$ D. Harper, ${ }^{86}$ R. D. Harrington, ${ }^{45}$ O. M. Harris, ${ }^{137}$ K. Harrison, ${ }^{17}$ J. Hartert, ${ }^{47}$ F. Hartjes ${ }^{104}$ T. Haruyama ${ }^{65}$ A. Harvey,${ }^{55}$ S. Hasegawa, ${ }^{100}$ Y. Hasegawa, ${ }^{139}$ S. Hassani, ${ }^{135}$ M. Hatch, ${ }^{29}$ D. Hauff, ${ }^{98}$ S. Haug, ${ }^{16}$ M. Hauschild, ${ }^{29}$ R. Hauser, ${ }^{87}$ M. Havranek, ${ }^{20}$ B. M. Hawes, ${ }^{117}$ C. M. Hawkes, ${ }^{17}$ R. J. Hawkings,${ }^{29}$ A. D. Hawkins, ${ }^{78}$ D. Hawkins, ${ }^{162}$ T. Hayakawa, ${ }^{66}$ T. Hayashi, ${ }^{159}$ D. Hayden, ${ }^{75}$ H. S. Hayward ${ }^{72}$ S. J. Haywood,${ }^{128}$ E. Hazen, ${ }^{21}$ M. He ${ }^{32 \mathrm{~d}}$ S. J. Head,${ }^{17}$ V. Hedberg, ${ }^{78}$ L. Heelan, ${ }^{7}$ S. Heim,${ }^{87}$ B. Heinemann, ${ }^{14}$ S. Heisterkamp,${ }^{35}$ L. Helary, ${ }^{4}$ C. Heller,${ }^{97}$ M. Heller ${ }^{29}$ S. Hellman, ${ }^{145 a, 145 b}$ D. Hellmich ${ }^{20}$ C. Helsens, ${ }^{11}$ R. C. W. Henderson, ${ }^{70}$ M. Henke, ${ }^{57 a}$ A. Henrichs, ${ }^{53}$ A. M. Henriques Correia, ${ }^{29}$ S. Henrot-Versille, ${ }^{114}$ F. Henry-Couannier, ${ }^{82}$ C. Hensel,${ }^{53}$ T. Hen $\beta,{ }^{173}$ C. M. Hernandez,${ }^{7}$ Y. Hernández Jiménez, ${ }^{166}$ R. Herrberg, ${ }^{15}$ A. D. Hershenhorn, ${ }^{151}$ G. Herten, ${ }^{47}$ R. Hertenberger, ${ }^{97}$ L. Hervas, ${ }^{29}$ N. P. Hessey, ${ }^{104}$ E. Higón-Rodriguez, ${ }^{166}$ D. Hill,,${ }^{5, a}$ J. C. Hill, ${ }^{27}$ N. Hill, ${ }^{5}$ K. H. Hiller ${ }^{41}$ S. Hillert, ${ }^{20}$ S. J. Hillier, ${ }^{17}$ I. Hinchliffe, ${ }^{14}$ E. Hines, ${ }^{119}$ M. Hirose,${ }^{115}$ F. Hirsch, ${ }^{42}$ D. Hirschbuehl, ${ }^{173}$ J. Hobbs, ${ }^{147}$ N. Hod,${ }^{152}$ M. C. Hodgkinson, ${ }^{138}$ P. Hodgson, ${ }^{138}$ A. Hoecker, ${ }^{29}$ M. R. Hoeferkamp, ${ }^{102}$ J. Hoffman, ${ }^{39}$ D. Hoffmann, ${ }^{82}$ M. Hohlfeld, ${ }^{80}$ M. Holder, ${ }^{140}$ S. O. Holmgren, ${ }^{145 a}$ T. Holy, ${ }^{126}$ J. L. Holzbauer, ${ }^{87}$ Y. Homma, ${ }^{66}$ T. M. Hong, ${ }^{119}$ L. Hooft van Huysduynen, ${ }^{107}$ T. Horazdovsky, ${ }^{126}$ C. Horn, ${ }^{142}$ S. Horner, ${ }^{47}$ J-Y. Hostachy, ${ }^{54}$ S. Hou, ${ }^{150}$ M. A. Houlden, ${ }^{72}$ A. Hoummada ${ }^{134 a}$ J. Howarth, ${ }^{81}$ D. F. Howell,,${ }^{117}$ I. Hristova, ${ }^{15}$ J. Hrivnac, ${ }^{114}$ I. Hruska, ${ }^{124}$ T. Hryn'ova, ${ }^{4}$ P. J. Hsu ${ }^{80}$ S.-C. Hsu, ${ }^{14}$ G. S. Huang,,${ }^{10}$ Z. Hubacek, ${ }^{126}$ F. Hubaut,${ }^{82}$ F. Huegging, ${ }^{20}$ A. Huettmann, ${ }^{41}$ T. B. Huffman, ${ }^{117}$ E. W. Hughes,${ }^{34}$ G. Hughes,${ }^{70}$ R. E. Hughes-Jones, ${ }^{81}$ M. Huhtinen, ${ }^{29}$ P. Hurst,${ }^{56}$ M. Hurwitz,${ }^{14}$

U. Husemann, ${ }^{41}$ N. Huseynov, ${ }^{64, o}$ J. Huston, ${ }^{87}$ J. Huth,${ }^{56}$ G. Iacobucci,${ }^{48}$ G. Iakovidis, ${ }^{9}$ M. Ibbotson, ${ }^{81}$

I. Ibragimov, ${ }^{140} \mathrm{R}$. Ichimiya, ${ }^{66} \mathrm{~L}$. Iconomidou-Fayard, ${ }^{114} \mathrm{~J}$. Idarraga, ${ }^{114} \mathrm{P}$. Iengo, ${ }^{101 \mathrm{a}, 101 \mathrm{~b}}$ O. Igonkina, ${ }^{104}$

Y. Ikegami ${ }^{65}$ M. Ikeno, ${ }^{65}$ Y. Ilchenko,${ }^{39}$ D. Iliadis, ${ }^{153}$ N. Ilic, ${ }^{157}$ D. Imbault,${ }^{77}$ M. Imori, ${ }^{154}$ T. Ince,${ }^{20}$ J. Inigo-Golfin, ${ }^{29}$ P. Ioannou, ${ }^{8} \mathrm{M}$. Iodice, ${ }^{133 a}$ V. Ippolito, ${ }^{131 \mathrm{a}, 131 \mathrm{~b}}$ A. Irles Quiles, ${ }^{166} \mathrm{C}$. Isaksson, ${ }^{165} \mathrm{~A}$. Ishikawa, ${ }^{66}$ M. Ishino, ${ }^{67} \mathrm{R}$. Ishmukhametov, ${ }^{39} \mathrm{C}$. Issever, ${ }^{117} \mathrm{~S}$. Istin, ${ }^{18 \mathrm{a}} \mathrm{A}$. V. Ivashin, ${ }^{127} \mathrm{~W}$. Iwanski, ${ }^{38} \mathrm{H}$. Iwasaki, ${ }^{65} \mathrm{~J}$. M. Izen, ${ }^{40}$ V. Izzo, ${ }^{101 a}$ B. Jackson, ${ }^{119}$ J. N. Jackson, ${ }^{72}$ P. Jackson, ${ }^{142}$ M. R. Jaekel, ${ }^{29}$ V. Jain, ${ }^{60}$ K. Jakobs,${ }^{47}$ S. Jakobsen, ${ }^{35}$ J. Jakubek, ${ }^{126}$ D. K. Jana, ${ }^{110}$ E. Jankowski, ${ }^{157}$ E. Jansen, ${ }^{76}$ H. Jansen, ${ }^{29}$ A. Jantsch,,${ }^{98}$ M. Janus, ${ }^{20}$ G. Jarlskog, ${ }^{78}$ L. Jeanty, ${ }^{56}$ K. Jelen, ${ }^{37}$ I. Jen-La Plante,${ }^{30}$ P. Jenni, ${ }^{29}$ A. Jeremie,,${ }^{4}$ P. Jež ${ }^{35}$ S. Jézéquel, ${ }^{4}$ M. K. Jha, ${ }^{19 a}$ H. Ji,${ }^{171}$ W. Ji, ${ }^{80}$ J. Jia, ${ }^{147}$ Y. Jiang, ${ }^{32 b}$ M. Jimenez Belenguer, ${ }^{41}$ G. Jin, ${ }^{32 b}$ S. Jin, ${ }^{32 a}$ O. Jinnouchi, ${ }^{156}$ M. D. Joergensen, ${ }^{35}$ D. Joffe, ${ }^{39}$ L. G. Johansen, ${ }^{13}$ M. Johansen, ${ }^{145 a, 145 b}$ K. E. Johansson, ${ }^{145 a}$ P. Johansson, ${ }^{138}$ S. Johnert, ${ }^{41}$ K. A. Johns, ${ }^{6}$ K. Jon-And, ${ }^{145 a, 145 b}$ G. Jones, ${ }^{81}$ R. W. L. Jones ${ }^{70}$ T. W. Jones ${ }^{76}$ T. J. Jones, ${ }^{72}$ O. Jonsson, ${ }^{29}$ C. Joram, ${ }^{29}$ P. M. Jorge, ${ }^{123 a}$ J. Joseph, ${ }^{14}$ T. Jovin, ${ }^{12 b}$ X. Ju, ${ }^{171}$ C. A. Jung ${ }^{42}$ R. M. Jungst,${ }^{29}$ V. Juranek, ${ }^{124}$ P. Jussel,,${ }^{61}$ A. Juste Rozas, ${ }^{11}$ V. V. Kabachenko, ${ }^{127}$ S. Kabana,${ }^{16}$ M. Kaci, ${ }^{166}$ A. Kaczmarska, ${ }^{38}$ P. Kadlecik, ${ }^{35}$ M. Kado,${ }^{114}$ H. Kagan, ${ }^{108}$ M. Kagan, ${ }^{56}$ S. Kaiser, ${ }^{98}$ E. Kajomovitz,${ }^{151}$ S. Kalinin, ${ }^{173}$ L. V. Kalinovskaya, ${ }^{64}$ S. Kama, ${ }^{39}$ N. Kanaya, ${ }^{154}$ M. Kaneda, ${ }^{29}$ S. Kaneti, ${ }^{27}$ T. Kanno, ${ }^{156}$ V. A. Kantserov, ${ }^{95}$ J. Kanzaki, ${ }^{65}$ B. Kaplan, ${ }^{174}$ A. Kapliy, ${ }^{30}$ J. Kaplon, ${ }^{29}$ D. Kar, ${ }^{43}$ M. Karagoz, ${ }^{117}$ M. Karnevskiy, ${ }^{41}$ K. Karr, ${ }^{5}$ V. Kartvelishvili, ${ }^{70}$ A. N. Karyukhin, ${ }^{127}$

L. Kashif, ${ }^{171}$ G. Kasieczka, ${ }^{57 b}$ A. Kasmi, ${ }^{39}$ R. D. Kass, ${ }^{108}$ A. Kastanas, ${ }^{13}$ M. Kataoka, ${ }^{4}$ Y. Kataoka, ${ }^{154}$ E. Katsoufis, ${ }^{9}$ J. Katzy, ${ }^{41}$ V. Kaushik, ${ }^{6}$ K. Kawagoe, ${ }^{66}$ T. Kawamoto, ${ }^{154}$ G. Kawamura, ${ }^{80}$ M. S. Kayl, ${ }^{104}$ V. A. Kazanin, ${ }^{106}$ M. Y. Kazarinov, ${ }^{64}$ R. Keeler, ${ }^{168}$ R. Kehoe,${ }^{39}$ M. Keil,${ }^{53}$ G. D. Kekelidze, ${ }^{64}$ J. Kennedy, ${ }^{97}$ C. J. Kenney,${ }^{142}$ M. Kenyon, ${ }^{52}$ O. Kepka, ${ }^{124}$ N. Kerschen, ${ }^{29}$ B. P. Kerševan, ${ }^{73}$ S. Kersten, ${ }^{173}$ K. Kessoku, ${ }^{154}$ J. Keung,,${ }^{157}$ M. Khakzad, ${ }^{28}$ F. Khalil-zada, ${ }^{10}$ H. Khandanyan, ${ }^{164}$ A. Khanov, ${ }^{111}$ D. Kharchenko, ${ }^{64}$ A. Khodinov, ${ }^{95}$ A. G. Kholodenko, ${ }^{127}$ A. Khomich, ${ }^{57 a}$ T. J. Khoo,${ }^{27}$ G. Khoriauli, ${ }^{20}$ A. Khoroshilov, ${ }^{173}$ N. Khovanskiy, ${ }^{64}$ 
V. Khovanskiy, ${ }^{94}$ E. Khramov, ${ }^{64}$ J. Khubua, ${ }^{50 b}$ H. Kim,,${ }^{145 a, 145 b}$ M. S. Kim, ${ }^{2}$ P. C. Kim, ${ }^{142}$ S. H. Kim,,${ }^{159}$ N. Kimura, ${ }^{169}$ O. Kind, ${ }^{15}$ B. T. King, ${ }^{72}$ M. King, ${ }^{66}$ R. S. B. King, ${ }^{117}$ J. Kirk, ${ }^{128}$ L. E. Kirsch,${ }^{22}$ A.E. Kiryunin,${ }^{98}$ T. Kishimoto, ${ }^{66}$ D. Kisielewska, ${ }^{37}$ T. Kittelmann, ${ }^{122}$ A. M. Kiver, ${ }^{127}$ E. Kladiva, ${ }^{143 b}$ J. Klaiber-Lodewigs, ${ }^{42}$ M. Klein, ${ }^{72}$ U. Klein, ${ }^{72}$ K. Kleinknecht,${ }^{80}$ M. Klemetti, ${ }^{84}$ A. Klier, ${ }^{170}$ P. Klimek, ${ }^{145 a, 145 b}$ A. Klimentov, ${ }^{24}$ R. Klingenberg, ${ }^{42}$ E. B. Klinkby, ${ }^{35}$ T. Klioutchnikova, ${ }^{29}$ P. F. Klok, ${ }^{103}$ S. Klous, ${ }^{104}$ E.-E. Kluge, ${ }^{57 a}$ T. Kluge, ${ }^{72}$ P. Kluit, ${ }^{104}$ S. Kluth, ${ }^{98}$ N. S. Knecht, ${ }^{157}$ E. Kneringer, ${ }^{61}$ J. Knobloch, ${ }^{29}$ E. B. F. G. Knoops, ${ }^{82}$ A. Knue, ${ }^{53}$ B. R. Ko, ${ }^{44}$ T. Kobayashi ${ }^{154}$ M. Kobel, ${ }^{43}$ M. Kocian, ${ }^{142}$ P. Kodys, ${ }^{125}$ K. Köneke, ${ }^{29}$ A. C. König, ${ }^{103}$ S. Koenig, ${ }^{80}$ L. Köpke, ${ }^{80}$ F. Koetsveld, ${ }^{103}$ P. Koevesarki, ${ }^{20}$ T. Koffas,${ }^{28}$ E. Koffeman, ${ }^{104}$ L. A. Kogan, ${ }^{117}$ F. Kohn,${ }^{53}$ Z. Kohout, ${ }^{126}$ T. Kohriki, ${ }^{65}$ T. Koi, ${ }^{142}$ T. Kokott, ${ }^{20}$ G. M. Kolachev, ${ }^{106}$ H. Kolanoski, ${ }^{15}$ V. Kolesnikov, ${ }^{64}$ I. Koletsou, ${ }^{88 a}$ J. Koll, ${ }^{87}$ D. Kollar, ${ }^{29}$

M. Kollefrath, ${ }^{47}$ S. D. Kolya, ${ }^{81}$ A. A. Komar, ${ }^{93}$ Y. Komori, ${ }^{154}$ T. Kondo, ${ }^{65}$ T. Kono, ${ }^{41, p}$ A. I. Kononov, ${ }^{47}$

R. Konoplich, ${ }^{107, q}$ N. Konstantinidis, ${ }^{76}$ A. Kootz, ${ }^{173}$ S. Koperny, ${ }^{37}$ K. Korcyl,${ }^{38}$ K. Kordas, ${ }^{153}$ V. Koreshev, ${ }^{127}$

A. Korn, ${ }^{117}$ A. Korol, ${ }^{106}$ I. Korolkov, ${ }^{11}$ E. V. Korolkova, ${ }^{138}$ V. A. Korotkov, ${ }^{127}$ O. Kortner, ${ }^{98}$ S. Kortner, ${ }^{98}$ V. V. Kostyukhin, ${ }^{20}$ M. J. Kotamäki, ${ }^{29}$ S. Kotov, ${ }^{98}$ V. M. Kotov, ${ }^{64}$ A. Kotwal, ${ }^{44}$ C. Kourkoumelis, ${ }^{8}$ V. Kouskoura, ${ }^{153}$ A. Koutsman, ${ }^{158 a}$ R. Kowalewski, ${ }^{168}$ T.Z. Kowalski, ${ }^{37}$ W. Kozanecki, ${ }^{135}$ A. S. Kozhin, ${ }^{127}$ V. Kral, ${ }^{126}$ V. A. Kramarenko, ${ }^{96}$ G. Kramberger, ${ }^{73}$ M. W. Krasny, ${ }^{77}$ A. Krasznahorkay, ${ }^{107}$ J. Kraus, ${ }^{87}$ J. K. Kraus,${ }^{20}$ A. Kreisel, ${ }^{152}$ F. Krejci, ${ }^{126}$ J. Kretzschmar, ${ }^{72}$ N. Krieger, ${ }^{53}$ P. Krieger, ${ }^{157}$ K. Kroeninger,${ }^{53}$ H. Kroha, ${ }^{98}$ J. Kroll, ${ }^{119}$ J. Kroseberg, ${ }^{20}$ J. Krstic, ${ }^{12 a}$ U. Kruchonak ${ }^{64}$ H. Krüger,${ }^{20}$ T. Kruker, ${ }^{16}$ N. Krumnack, ${ }^{63}$ Z. V. Krumshteyn, ${ }^{64}$ A. Kruth,${ }^{20}$ T. Kubota ${ }^{85}$ S. Kuehn, ${ }^{47}$ A. Kugel, ${ }^{57 \mathrm{c}}$ T. Kuhl, ${ }^{41}$ V. Kukhtin, ${ }^{64}$ Y. Kulchitsky, ${ }^{89}$ S. Kuleshov, ${ }^{31 \mathrm{~b}}$ C. Kummer, ${ }^{97}$ M. Kuna, ${ }^{77}$ N. Kundu, ${ }^{117}$ J. Kunkle, ${ }^{119}$ A. Kupco, ${ }^{124}$ H. Kurashige, ${ }^{66}$ M. Kurata, ${ }^{159}$ Y. A. Kurochkin,${ }^{89}$ V. Kus, ${ }^{124}$ E. S. Kuwertz, ${ }^{146}$ M. Kuze, ${ }^{156}$ J. Kvita, ${ }^{141}$ R. Kwee, ${ }^{15}$ A. La Rosa ${ }^{48}$ L. La Rotonda, ${ }^{36 a, 36 b}$ L. Labarga, ${ }^{79}$ J. Labbe, ${ }^{4}$ S. Lablak, ${ }^{134 a}$ C. Lacasta, ${ }^{166}$ F. Lacava, ${ }^{131 a, 131 b}$ H. Lacker, ${ }^{15}$ D. Lacour, ${ }^{77}$ V. R. Lacuesta, ${ }^{166}$ E. Ladygin,${ }^{64}$ R. Lafaye, ${ }^{4}$ B. Laforge,${ }^{77}$ T. Lagouri, ${ }^{79}$ S. Lai, ${ }^{47}$ E. Laisne,${ }^{54}$ M. Lamanna, ${ }^{29}$ C. L. Lampen, ${ }^{6}$ W. Lampl,${ }^{6}$ E. Lancon, ${ }^{135}$ U. Landgraf, ${ }^{47}$ M. P. J. Landon, ${ }^{74}$ H. Landsman, ${ }^{151}$ J. L. Lane,${ }^{81}$ C. Lange, ${ }^{41}$ A. J. Lankford, ${ }^{162}$ F. Lanni, ${ }^{24}$ K. Lantzsch, ${ }^{173}$ S. Laplace,${ }^{77}$ C. Lapoire, ${ }^{20}$ J. F. Laporte, ${ }^{135}$ T. Lari, ${ }^{88 a}$ A. V. Larionov, ${ }^{127}$ A. Larner, ${ }^{117}$ C. Lasseur, ${ }^{29}$ M. Lassnig, ${ }^{29}$ P. Laurelli, ${ }^{46}$ W. Lavrijsen, ${ }^{14}$ P. Laycock, ${ }^{72}$ A. B. Lazarev, ${ }^{64}$ O. Le Dortz, ${ }^{77}$ E. Le Guirriec, ${ }^{82}$ C. Le Maner, ${ }^{157}$ E. Le Menedeu, ${ }^{9}$ C. Lebel, ${ }^{92}$ T. LeCompte, ${ }^{5}$ F. Ledroit-Guillon,${ }^{54}$ H. Lee, ${ }^{104}$ J. S. H. Lee, ${ }^{115}$ S. C. Lee, ${ }^{150}$ L. Lee, ${ }^{174}$ M. Lefebvre, ${ }^{168}$ M. Legendre, ${ }^{135}$ A. Leger, ${ }^{48}$ B. C. LeGeyt, ${ }^{119}$ F. Legger, ${ }^{97}$ C. Leggett, ${ }^{14}$ M. Lehmacher, ${ }^{20}$ G. Lehmann Miotto, ${ }^{29}$ X. Lei, ${ }^{6}$ M. A. L. Leite, ${ }^{23 \mathrm{~d}}$ R. Leitner, ${ }^{125}$ D. Lellouch, ${ }^{170}$ M. Leltchouk, ${ }^{34}$ B. Lemmer, ${ }^{53}$ V. Lendermann, ${ }^{57 a}$ K. J. C. Leney, ${ }^{144 b}$ T. Lenz, ${ }^{104}$ G. Lenzen, ${ }^{173}$ B. Lenzi, ${ }^{29}$ K. Leonhardt, ${ }^{43}$ S. Leontsinis, ${ }^{9}$ C. Leroy, ${ }^{92}$ J-R. Lessard, ${ }^{168}$ J. Lesser, ${ }^{145 a}$ C. G. Lester, ${ }^{27}$ A. Leung Fook Cheong, ${ }^{171}$ J. Levêque, ${ }^{4}$ D. Levin, ${ }^{86}$ L. J. Levinson, ${ }^{170}$ M. S. Levitski, ${ }^{127}$ A. Lewis, ${ }^{117}$ G. H. Lewis, ${ }^{107}$ A. M. Leyko, ${ }^{20}$ M. Leyton, ${ }^{15}$ B. Li ${ }^{82}$ H. Li ${ }^{171}$ S. Li,${ }^{32 b, r}$ X. Li,${ }^{86}$ Z. Liang, ${ }^{117, s}$ H. Liao,${ }^{33}$ B. Liberti, ${ }^{132 a}$ P. Lichard, ${ }^{29}$ M. Lichtnecker ${ }^{97}$ K. Lie, ${ }^{164}$ W. Liebig, ${ }^{13}$ R. Lifshitz, ${ }^{151}$ J. N. Lilley, ${ }^{17}$ C. Limbach, ${ }^{20}$ A. Limosani,${ }^{85}$ M. Limper,${ }^{62}$ S. C. Lin ${ }^{150, t}$ F. Linde, ${ }^{104}$ J. T. Linnemann,${ }^{87}$ E. Lipeles, ${ }^{119}$ L. Lipinsky,${ }^{124}$ A. Lipniacka, ${ }^{13}$ T. M. Liss,${ }^{164}$ D. Lissauer, ${ }^{24}$ A. Lister, ${ }^{48}$ A. M. Litke, ${ }^{136}$ C. Liu, ${ }^{28}$ D. Liu, ${ }^{150}$ H. Liu, ${ }^{86}$ J. B. Liu, ${ }^{86}$ M. Liu, ${ }^{32 b}$ S. Liu, ${ }^{2}$ Y. Liu, ${ }^{32 b}$ M. Livan, ${ }^{118 a, 118 b}$ S. S. A. Livermore, ${ }^{117}$ A. Lleres,${ }^{54}$ J. Llorente Merino ${ }^{79}$ S. L. Lloyd ${ }^{74}$ E. Lobodzinska, ${ }^{41}$ P. Loch, ${ }^{6}$ W. S. Lockman, ${ }^{136}$ T. Loddenkoetter, ${ }^{20}$ F. K. Loebinger, ${ }^{81}$ A. Loginov, ${ }^{174}$ C. W. Loh, ${ }^{167}$ T. Lohse, ${ }^{15}$ K. Lohwasser ${ }^{47}$ M. Lokajicek, ${ }^{124}$ J. Loken, ${ }^{17}$ V. P. Lombardo, ${ }^{4}$ R. E. Long, ${ }^{70}$ L. Lopes, ${ }^{123 a, c}$ D. Lopez Mateos,${ }^{56}$ J. Lorenz, ${ }^{97}$ M. Losada, ${ }^{161}$ P. Loscutoff, ${ }^{14}$ F. Lo Sterzo, ${ }^{131 \mathrm{a}, 131 \mathrm{~b}}$ M. J. Losty,${ }^{158 \mathrm{a}}$ X. Lou ${ }^{40}$ A. Lounis, ${ }^{114}$ K. F. Loureiro, ${ }^{161}$ J. Love, ${ }^{21}$ P. A. Love, ${ }^{70}$ A. J. Lowe, ${ }^{142, f}$ F. Lu, ${ }^{32 a}$ H. J. Lubatti, ${ }^{137}$ C. Luci, ${ }^{131 a, 131 b}$ A. Lucotte, ${ }^{54}$ A. Ludwig, ${ }^{43}$ D. Ludwig, ${ }^{41}$ I. Ludwig, ${ }^{47}$ J. Ludwig, ${ }^{47}$ F. Luehring, ${ }^{60}$ G. Luijckx, ${ }^{104}$ D. Lumb,${ }^{47}$ L. Luminari, ${ }^{131 a}$ E. Lund, ${ }^{116}$ B. Lund-Jensen, ${ }^{146}$ B. Lundberg, ${ }^{78}$ J. Lundberg, ${ }^{145 a, 145 b}$ J. Lundquist,${ }^{35}$ M. Lungwitz, ${ }^{80}$ G. Lutz, ${ }^{98}$ D. Lynn, ${ }^{24}$ J. Lys, ${ }^{14}$ E. Lytken, ${ }^{78}$ H. Ma, ${ }^{24}$ L. L. Ma,${ }^{171}$ J. A. Macana Goia, ${ }^{92}$ G. Maccarrone, ${ }^{46}$ A. Macchiolo, ${ }^{98}$ B. Maček,${ }^{73}$ J. Machado Miguens, ${ }^{123 a}$ R. Mackeprang, ${ }^{35}$ R. J. Madaras, ${ }^{14}$ W. F. Mader, ${ }^{43}$ R. Maenner, ${ }^{57 c}$ T. Maeno, ${ }^{24}$ P. Mättig, ${ }^{173}$ S. Mättig, ${ }^{41}$ L. Magnoni, ${ }^{29}$ E. Magradze,${ }^{53}$ Y. Mahalalel, ${ }^{152}$ K. Mahboubi, ${ }^{47}$ G. Mahout, ${ }^{17}$ C. Maiani, ${ }^{131 a, 131 b}$ C. Maidantchik, ${ }^{23 a}$ A. Maio, ${ }^{123 a, c}$ S. Majewski, ${ }^{24}$ Y. Makida, ${ }^{65}$ N. Makovec, ${ }^{114}$ P. Mal, ${ }^{135}$ B. Malaescu, ${ }^{29}$ Pa. Malecki, ${ }^{38}$ P. Malecki, ${ }^{38}$ V. P. Maleev, ${ }^{120}$ F. Malek,${ }^{54}$ U. Mallik, ${ }^{62}$ D. Malon, ${ }^{5}$ C. Malone,${ }^{142}$ S. Maltezos,${ }^{9}$ V. Malyshev, ${ }^{106}$ S. Malyukov, ${ }^{29}$ R. Mameghani ${ }^{97}$ J. Mamuzic, ${ }^{12 b}$ A. Manabe,${ }^{65}$ L. Mandelli, ${ }^{88 a}$ I. Mandić, ${ }^{73}$ R. Mandrysch, ${ }^{15}$ J. Maneira, ${ }^{123 a}$ P. S. Mangeard, ${ }^{87}$ L. Manhaes de Andrade Filho, ${ }^{23 a}$ I. D. Manjavidze, ${ }^{64}$ A. Mann, ${ }^{53}$ P. M. Manning, ${ }^{136}$ A. Manousakis-Katsikakis, ${ }^{8}$ B. Mansoulie, ${ }^{135}$ A. Manz,${ }^{98}$ A. Mapelli, ${ }^{29}$ L. Mapelli, ${ }^{29}$ L. March, ${ }^{79}$ J.F. Marchand, ${ }^{28}$ F. Marchese, ${ }^{132 a, 1323 b}$ G. Marchiori, ${ }^{77}$ M. Marcisovsky, ${ }^{124}$ A. Marin, ${ }^{21, a}$ C. P. Marino, ${ }^{168}$ 
F. Marroquim, ${ }^{23 a}$ R. Marshall, ${ }^{81}$ Z. Marshall, ${ }^{29}$ F. K. Martens, ${ }^{157}$ S. Marti-Garcia, ${ }^{166}$ A. J. Martin, ${ }^{174}$ B. Martin, ${ }^{29}$ B. Martin, ${ }^{87}$ F. F. Martin, ${ }^{119}$ J. P. Martin, ${ }^{92}$ Ph. Martin, ${ }^{54}$ T. A. Martin, ${ }^{17}$ V. J. Martin, ${ }^{45}$ B. Martin dit Latour, ${ }^{48}$ S. Martin-Haugh, ${ }^{148}$ M. Martinez,${ }^{11}$ V. Martinez Outschoorn ${ }^{56}$ A. C. Martyniuk, ${ }^{168}$ M. Marx,${ }^{81}$ F. Marzano, ${ }^{131 a}$ A. Marzin, ${ }^{110}$ L. Masetti, ${ }^{80}$ T. Mashimo, ${ }^{154}$ R. Mashinistov, ${ }^{93}$ J. Masik, ${ }^{81}$ A. L. Maslennikov, ${ }^{106}$ I. Massa, ${ }^{19 a, 19 b}$ G. Massaro, ${ }^{104}$ N. Massol, ${ }^{4}$ P. Mastrandrea, ${ }^{131 a, 131 b}$ A. Mastroberardino, ${ }^{36 a, 36 b}$ T. Masubuchi, ${ }^{154}$ M. Mathes,${ }^{20}$

H. Matsumoto, ${ }^{154}$ H. Matsunaga, ${ }^{154}$ T. Matsushita, ${ }^{66}$ C. Mattravers, ${ }^{117, d}$ J. M. Maugain, ${ }^{29}$ J. Maurer, ${ }^{82}$

S. J. Maxfield, ${ }^{72}$ D. A. Maximov, ${ }^{106}$ E. N. May, ${ }^{5}$ A. Mayne, ${ }^{138}$ R. Mazini, ${ }^{150}$ M. Mazur, ${ }^{20}$ M. Mazzanti, ${ }^{88 a}$ E. Mazzoni, ${ }^{121 a, 121 b}$ S. P. Mc Kee, ${ }^{86}$ A. McCarn, ${ }^{164}$ R. L. McCarthy, ${ }^{147}$ T. G. McCarthy, ${ }^{28}$ N. A. McCubbin, ${ }^{128}$ K. W. McFarlane, ${ }^{55}$ J. A. Mcfayden, ${ }^{138}$ H. McGlone, ${ }^{52}$ G. Mchedlidze, ${ }^{50 b}$ R. A. McLaren, ${ }^{29}$ T. Mclaughlan, ${ }^{17}$ S. J. McMahon, ${ }^{128}$ R. A. McPherson, ${ }^{168, j}$ A. Meade, ${ }^{83}$ J. Mechnich, ${ }^{104}$ M. Mechtel,${ }^{173}$ M. Medinnis, ${ }^{41}$ R. Meera-Lebbai, ${ }^{110}$ T. Meguro, ${ }^{115}$ R. Mehdiyev, ${ }^{92}$ S. Mehlhase, ${ }^{35}$ A. Mehta, ${ }^{72}$ K. Meier,${ }^{57 a}$ B. Meirose,${ }^{78}$ C. Melachrinos, ${ }^{30}$ B. R. Mellado Garcia, ${ }^{171}$ L. Mendoza Navas, ${ }^{161}$ Z. Meng, ${ }^{150, \mathrm{u}}$ A. Mengarelli, ${ }^{19 a, 19 b}$ S. Menke, ${ }^{98}$ C. Menot ${ }^{29}$ E. Meoni, ${ }^{11}$ K. M. Mercurio, ${ }^{56}$ P. Mermod, ${ }^{48}$ L. Merola, ${ }^{101 a, 101 b}$ C. Meroni, ${ }^{88 a}$ F. S. Merritt, ${ }^{30}$ A. Messina, ${ }^{29}$ J. Metcalfe, ${ }^{102}$ A. S. Mete, ${ }^{63}$ C. Meyer, ${ }^{80}$ C. Meyer, ${ }^{30}$ J-P. Meyer, ${ }^{135}$ J. Meyer, ${ }^{172}$ J. Meyer, ${ }^{53}$ T. C. Meyer, ${ }^{29}$ W. T. Meyer, ${ }^{63}$ J. Miao, ${ }^{32 d}$ S. Michal, ${ }^{29}$ L. Micu, ${ }^{25 a}$ R. P. Middleton, ${ }^{128}$ S. Migas, ${ }^{72}$ L. Mijović,${ }^{41}$ G. Mikenberg, ${ }^{170}$ M. Mikestikova, ${ }^{124}$ M. Mikuž, ${ }^{73}$ D. W. Miller, ${ }^{30}$ R. J. Miller, ${ }^{87}$ W. J. Mills, ${ }^{167}$ C. Mills, ${ }^{56}$ A. Milov, ${ }^{170}$ D. A. Milstead, ${ }^{145 a, 145 b}$ D. Milstein, ${ }^{170}$ A. A. Minaenko, ${ }^{127}$ M. Miñano Moya, ${ }^{166}$ I. A. Minashvili, ${ }^{64}$ A. I. Mincer, ${ }^{107}$ B. Mindur, ${ }^{37}$ M. Mineev, ${ }^{64}$ Y. Ming, ${ }^{171}$ L. M. Mir, ${ }^{11}$ G. Mirabelli, ${ }^{131 a}$ L. Miralles Verge,${ }^{11}$ A. Misiejuk, ${ }^{75}$ J. Mitrevski, ${ }^{136}$ G. Y. Mitrofanov, ${ }^{127}$ V. A. Mitsou, ${ }^{166}$ S. Mitsui, ${ }^{65}$ P. S. Miyagawa ${ }^{138}$ K. Miyazaki, ${ }^{66}$ J. U. Mjörnmark, ${ }^{78}$ T. Moa, ${ }^{145 a, 145 b}$ P. Mockett, ${ }^{137}$ S. Moed,${ }^{56}$ V. Moeller, ${ }^{27}$ K. Mönig, ${ }^{41}$ N. Möser,${ }^{20}$ S. Mohapatra ${ }^{147}$ W. Mohr ${ }^{47}$ S. Mohrdieck-Möck, ${ }^{98}$ A. M. Moisseev, ${ }^{127, a}$ R. Moles-Valls, ${ }^{166}$ J. Molina-Perez, ${ }^{29}$ J. Monk ${ }^{76}$ E. Monnier, ${ }^{82}$ S. Montesano, ${ }^{88 a, 88 b}$ F. Monticelli, ${ }^{69}$ S. Monzani, ${ }^{19 a, 19 b}$ R. W. Moore, ${ }^{2}$ G. F. Moorhead ${ }^{85}$ C. Mora Herrera, ${ }^{48}$ A. Moraes ${ }^{52}$ N. Morange, ${ }^{135}$ J. Morel,${ }^{53}$ G. Morello, ${ }^{36 a, 36 b}$ D. Moreno, ${ }^{80}$ M. Moreno Llácer, ${ }^{166}$ P. Morettini, ${ }^{49 a}$ M. Morii, ${ }^{56}$ J. Morin,${ }^{74}$ A. K. Morley, ${ }^{29}$ G. Mornacchi, ${ }^{29}$ S. V. Morozov, ${ }^{95}$ J. D. Morris,${ }^{74}$ L. Morvaj, ${ }^{100}$ H. G. Moser, ${ }^{98}$ M. Mosidze, ${ }^{50 b}$ J. Moss, ${ }^{108}$ R. Mount, ${ }^{142}$ E. Mountricha, ${ }^{9}$ S. V. Mouraviev, ${ }^{93}$ E. J. W. Moyse, ${ }^{83}$ M. Mudrinic, ${ }^{12 b}$ F. Mueller, ${ }^{57 a}$ J. Mueller, ${ }^{122}$ K. Mueller, ${ }^{20}$ T. A. Müller, ${ }^{97}$ T. Mueller, ${ }^{80}$ D. Muenstermann, ${ }^{29}$ A. Muir, ${ }^{167}$ Y. Munwes, ${ }^{152}$ W. J. Murray, ${ }^{128}$ I. Mussche, ${ }^{104}$ E. Musto, ${ }^{101 a, 101 b}$ A. G. Myagkov, ${ }^{127}$ J. Nadal, ${ }^{11}$ K. Nagai, ${ }^{159}$ K. Nagano, ${ }^{65}$ Y. Nagasaka,${ }^{59}$ M. Nagel,${ }^{98}$ A. M. Nairz ${ }^{29}$ Y. Nakahama, ${ }^{29}$ K. Nakamura,${ }^{154}$ T. Nakamura, ${ }^{154}$ I. Nakano, ${ }^{109}$ G. Nanava,${ }^{20}$ A. Napier ${ }^{160}$ R. Narayan, ${ }^{57 b}$ M. Nash, ${ }^{76, d}$ N. R. Nation, ${ }^{21}$

T. Nattermann, ${ }^{20}$ T. Naumann,${ }^{41}$ G. Navarro, ${ }^{161}$ H. A. Neal,${ }^{86}$ E. Nebot,${ }^{79}$ P. Yu. Nechaeva, ${ }^{93}$ A. Negri,,${ }^{18 a, 118 b}$ G. Negri, ${ }^{29}$ S. Nektarijevic, ${ }^{48}$ A. Nelson, ${ }^{162}$ S. Nelson, ${ }^{142}$ T. K. Nelson, ${ }^{142}$ S. Nemecek, ${ }^{124}$ P. Nemethy,,${ }^{107}$ A. A. Nepomuceno, ${ }^{23 a}$ M. Nessi,,${ }^{29, v}$ M. S. Neubauer, ${ }^{164}$ A. Neusiedl,${ }^{80}$ R. M. Neves, ${ }^{107}$ P. Nevski, ${ }^{24}$ P. R. Newman, ${ }^{17}$ V. Nguyen Thi Hong, ${ }^{135}$ R. B. Nickerson, ${ }^{117}$ R. Nicolaidou, ${ }^{135}$ L. Nicolas, ${ }^{138}$ B. Nicquevert, ${ }^{29}$ F. Niedercorn, ${ }^{114}$ J. Nielsen, ${ }^{136}$ T. Niinikoski, ${ }^{29}$ N. Nikiforou, ${ }^{34}$ A. Nikiforov, ${ }^{15}$ V. Nikolaenko, ${ }^{127}$ K. Nikolaev, ${ }^{64}$ I. Nikolic-Audit, ${ }^{77}$

K. Nikolics, ${ }^{48}$ K. Nikolopoulos,${ }^{24}$ H. Nilsen, ${ }^{47}$ P. Nilsson, ${ }^{7}$ Y. Ninomiya,${ }^{154}$ A. Nisati, ${ }^{131 a}$ T. Nishiyama, ${ }^{66}$ R. Nisius, ${ }^{98}$ L. Nodulman, ${ }^{5}$ M. Nomachi, ${ }^{115}$ I. Nomidis, ${ }^{153}$ M. Nordberg, ${ }^{29}$ B. Nordkvist, ${ }^{145 a, 145 b}$ P. R. Norton, ${ }^{128}$ J. Novakova, ${ }^{125}$ M. Nozaki, ${ }^{65}$ L. Nozka, ${ }^{112}$ I. M. Nugent, ${ }^{158 a}$ A.-E. Nuncio-Quiroz,${ }^{20}$ G. Nunes Hanninger, ${ }^{85}$ T. Nunnemann, ${ }^{97}$ E. Nurse, ${ }^{76}$ T. Nyman, ${ }^{29}$ B. J. O’Brien, ${ }^{45}$ S. W. O’Neale, ${ }^{17, a}$ D. C. O'Neil, ${ }^{141}$ V. O'Shea, ${ }^{52}$ L. B. Oakes, ${ }^{97}$ F. G. Oakham, ${ }^{28, e}$ H. Oberlack,${ }^{98}$ J. Ocariz,${ }^{77}$ A. Ochi,${ }^{66}$ S. Oda,${ }^{154}$ S. Odaka, ${ }^{65}$ J. Odier, ${ }^{82}$ H. Ogren, ${ }^{60}$ A. Oh, ${ }^{81}$ S. H. Oh, ${ }^{44}$ C. C. Ohm, ${ }^{145 a, 145 b}$ T. Ohshima, ${ }^{100}$ H. Ohshita, ${ }^{139}$ T. Ohsugi, ${ }^{58}$ S. Okada, ${ }^{66}$ H. Okawa, ${ }^{162}$ Y. Okumura ${ }^{100}$ T. Okuyama, ${ }^{154}$ A. Olariu, ${ }^{25 a}$ M. Olcese,${ }^{49 a}$ A. G. Olchevski, ${ }^{64}$ M. Oliveira,,${ }^{123 a, h}$

D. Oliveira Damazio, ${ }^{24}$ E. Oliver Garcia, ${ }^{166}$ D. Olivito, ${ }^{119}$ A. Olszewski, ${ }^{38}$ J. Olszowska, ${ }^{38}$ C. Omachi, ${ }^{66}$ A. Onofre, ${ }^{123 a, w}$ P. U. E. Onyisi, ${ }^{30}$ C. J. Oram, ${ }^{158 a}$ M. J. Oreglia, ${ }^{30}$ Y. Oren, ${ }^{152}$ D. Orestano, ${ }^{133 a, 133 b}$ C. Oropeza Barrera, ${ }^{52}$ R. S. Orr, ${ }^{157}$ B. Osculati, ${ }^{49 a, 49 b}$ R. Ospanov, ${ }^{19}$ C. Osuna, ${ }^{11}$ G. Otero y Garzon, ${ }^{26}$ J. P. Ottersbach, ${ }^{104}$ M. Ouchrif, ${ }^{134 d}$ E. A. Ouellette, ${ }^{168}$ F. Ould-Saada, ${ }^{116}$ A. Ouraou, ${ }^{135}$ Q. Ouyang, ${ }^{32 a}$ A. Ovcharova, ${ }^{14}$ M. Owen, ${ }^{81}$ S. Owen,,${ }^{138}$ V. E. Ozcan, ${ }^{18 a}$ N. Ozturk, ${ }^{7}$ A. Pacheco Pages, ${ }^{11}$ C. Padilla Aranda, ${ }^{11}$ S. Pagan Griso, ${ }^{14}$ E. Paganis, ${ }^{138}$ F. Paige,${ }^{24}$ P. Pais,${ }^{83}$ K. Pajchel, ${ }^{116}$ G. Palacino, ${ }^{158 b}$ C. P. Paleari, ${ }^{6}$ S. Palestini, ${ }^{29}$ D. Pallin, ${ }^{33}$ A. Palma,${ }^{123 a}$ J. D. Palmer, ${ }^{17}$ Y. B. Pan, ${ }^{171}$ E. Panagiotopoulou, ${ }^{9}$ B. Panes, ${ }^{31 a}$ N. Panikashvili,${ }^{86}$ S. Panitkin, ${ }^{24}$ D. Pantea, ${ }^{25 a}$ M. Panuskova, ${ }^{124}$ V. Paolone, ${ }^{122}$ A. Papadelis, ${ }^{145 a}$ Th. D. Papadopoulou, ${ }^{9}$ A. Paramonov, ${ }^{5}$ W. Park, ${ }^{24, \mathrm{x}}$ M. A. Parker, ${ }^{27}$ F. Parodi,${ }^{49 a, 49 b}$ J. A. Parsons, ${ }^{34}$ U. Parzefall,${ }^{47}$ E. Pasqualucci, ${ }^{131 a}$ S. Passaggio, ${ }^{49 a}$

A. Passeri, ${ }^{133 a}$ F. Pastore,${ }^{133 a, 133 b}$ Fr. Pastore,${ }^{75}$ G. Pásztor,${ }^{48, y}$ S. Pataraia,${ }^{173}$ N. Patel, ${ }^{149}$ J. R. Pater, ${ }^{81}$ 
S. Patricelli, ${ }^{101 a, 101 b}$ T. Pauly, ${ }^{29}$ M. Pecsy, ${ }^{143 a}$ M. I. Pedraza Morales, ${ }^{171}$ S. V. Peleganchuk, ${ }^{106}$ H. Peng,${ }^{32 b}$ R. Pengo, ${ }^{29}$ A. Penson, ${ }^{34}$ J. Penwell, ${ }^{60}$ M. Perantoni, ${ }^{23 a}$ K. Perez,${ }^{34, z}$ T. Perez Cavalcanti, ${ }^{41}$ E. Perez Codina, ${ }^{11}$ M. T. Pérez García-Estañ, ${ }^{166}$ V. Perez Reale,${ }^{34}$ L. Perini, ${ }^{88 a, 88 b}$ H. Pernegger,${ }^{29}$ R. Perrino, ${ }^{71 a}$ P. Perrodo, ${ }^{4}$ S. Persembe, ${ }^{3 a}$ V. D. Peshekhonov,${ }^{64}$ B. A. Petersen,${ }^{29}$ J. Petersen,${ }^{29}$ T. C. Petersen,${ }^{35}$ E. Petit,${ }^{4}$ A. Petridis, ${ }^{153}$ C. Petridou, ${ }^{153}$ E. Petrolo, ${ }^{131 a}$ F. Petrucci, ${ }^{133 a, 133 b}$ D. Petschull, ${ }^{41}$ M. Petteni,${ }^{141}$ R. Pezoa, ${ }^{31 b}$ A. Phan,${ }^{85}$ P. W. Phillips, ${ }^{128}$ G. Piacquadio, ${ }^{29}$ E. Piccaro, ${ }^{74}$ M. Piccinini, ${ }^{19 a, 19 b}$ S. M. Piec, ${ }^{41}$ R. Piegaia, ${ }^{26}$ D. T. Pignotti, ${ }^{108}$ J. E. Pilcher, ${ }^{30}$ A. D. Pilkington, ${ }^{81}$ J. Pina, ${ }^{123 a, c}$ M. Pinamonti, ${ }^{163 a, 163 c}$ A. Pinder, ${ }^{117}$ J. L. Pinfold, ${ }^{2}$ J. Ping, ${ }^{32 c}$ B. Pinto, ${ }^{123 a, c}$ O. Pirotte, ${ }^{29}$ C. Pizio, ${ }^{88 a, 88 b}$ R. Placakyte, ${ }^{41}$ M. Plamondon, ${ }^{168}$ M.-A. Pleier, ${ }^{24}$ A. V. Pleskach, ${ }^{127}$ A. Poblaguev,${ }^{24}$ S. Poddar,${ }^{57 a}$ F. Podlyski, ${ }^{33}$ L. Poggioli, ${ }^{114}$ T. Poghosyan,${ }^{20}$ M. Pohl, ${ }^{48}$ F. Polci, ${ }^{54}$ G. Polesello, ${ }^{118 a}$ A. Policicchio, ${ }^{137}$ A. Polini, ${ }^{19 a}$ J. Poll, ${ }^{74}$ V. Polychronakos,${ }^{24}$ D. M. Pomarede, ${ }^{135}$ D. Pomeroy,${ }^{22}$ K. Pommès, ${ }^{29}$

L. Pontecorvo, ${ }^{131 a}$ B. G. Pope,${ }^{87}$ G. A. Popeneciu, ${ }^{25 a}$ D. S. Popovic, ${ }^{12 a}$ A. Poppleton, ${ }^{29}$ X. Portell Bueso, ${ }^{29}$ C. Posch, ${ }^{21}$ G. E. Pospelov, ${ }^{98}$ S. Pospisil, ${ }^{126}$ I. N. Potrap,${ }^{98}$ C. J. Potter,${ }^{148}$ C. T. Potter,${ }^{113}$ G. Poulard, ${ }^{29}$ J. Poveda, ${ }^{171}$ R. Prabhu, ${ }^{76}$ P. Pralavorio, ${ }^{82}$ A. Pranko, ${ }^{14}$ S. Prasad,${ }^{56}$ R. Pravahan, ${ }^{7}$ S. Prell,${ }^{63} \mathrm{~K}$. Pretzl,${ }^{16}$ L. Pribyl, ${ }^{29}$ D. Price, ${ }^{60}$ J. Price, ${ }^{72}$ L. E. Price,${ }^{5}$ M. J. Price, ${ }^{29}$ D. Prieur, ${ }^{122}$ M. Primavera, ${ }^{71 \mathrm{a}}$ K. Prokofiev, ${ }^{107}$ F. Prokoshin,${ }^{31 \mathrm{~b}}$ S. Protopopescu, ${ }^{24}$ J. Proudfoot, ${ }^{5}$ X. Prudent, ${ }^{43}$ M. Przybycien, ${ }^{37}$ H. Przysiezniak, ${ }^{4}$ S. Psoroulas, ${ }^{20}$ E. Ptacek, ${ }^{113}$ E. Pueschel, ${ }^{83}$ J. Purdham, ${ }^{86}$ M. Purohit, ${ }^{24, x}$ P. Puzo, ${ }^{114}$ Y. Pylypchenko, ${ }^{62}$ J. Qian, ${ }^{86}$ Z. Qian, ${ }^{82}$ Z. Qin, ${ }^{41}$ A. Quadt,${ }^{53}$ D. R. Quarrie, ${ }^{14}$ W. B. Quayle, ${ }^{171}$ F. Quinonez, ${ }^{31 \mathrm{a}}$ M. Raas, ${ }^{103}$ V. Radescu, ${ }^{57 b}$ B. Radics, ${ }^{20}$ P. Radloff, ${ }^{113}$ T. Rador, ${ }^{18 a}$ F. Ragusa, ${ }^{88 a, 88 b}$ G. Rahal, ${ }^{176}$ A. M. Rahimi, ${ }^{108}$ D. Rahm, ${ }^{24}$ S. Rajagopalan, ${ }^{24}$ M. Rammensee, ${ }^{47}$ M. Rammes,${ }^{140}$ A. S. Randle-Conde ${ }^{39}$ K. Randrianarivony, ${ }^{28}$ P. N. Ratoff, ${ }^{70}$ F. Rauscher, ${ }^{97}$ M. Raymond, ${ }^{29}$ A. L. Read, ${ }^{116}$

D. M. Rebuzzi, ${ }^{118 \mathrm{a}, 118 \mathrm{~b}}$ A. Redelbach, ${ }^{172}$ G. Redlinger, ${ }^{24}$ R. Reece, ${ }^{119} \mathrm{~K}$. Reeves, ${ }^{40}$ A. Reichold,${ }^{104}$

E. Reinherz-Aronis, ${ }^{152}$ A. Reinsch, ${ }^{113}$ I. Reisinger, ${ }^{42}$ D. Reljic, ${ }^{12 a}$ C. Rembser,${ }^{29}$ Z. L. Ren ${ }^{150}$ A. Renaud, ${ }^{114}$ P. Renkel, ${ }^{39}$ M. Rescigno, ${ }^{131 a}$ S. Resconi ${ }^{88 a}$ B. Resende, ${ }^{135}$ P. Reznicek,${ }^{97}$ R. Rezvani, ${ }^{157}$ A. Richards, ${ }^{76}$ R. Richter, ${ }^{98}$ E. Richter-Was, ${ }^{4, \text { aa }}$ M. Ridel,${ }^{77}$ M. Rijpstra, ${ }^{104}$ M. Rijssenbeek, ${ }^{147}$ A. Rimoldi, ${ }^{118 a, 118 b}$ L. Rinaldi, ${ }^{19 a}$ R. R. Rios, ${ }^{39}$ I. Riu, ${ }^{11}$ G. Rivoltella, ${ }^{88 a, 88 b}$ F. Rizatdinova, ${ }^{111}$ E. Rizvi, ${ }^{74}$ S. H. Robertson, ${ }^{84, j}$ A. Robichaud-Veronneau, ${ }^{117}$ D. Robinson, ${ }^{27}$ J. E. M. Robinson, ${ }^{76}$ M. Robinson, ${ }^{113}$ A. Robson, ${ }^{52}$ J. G. Rocha de Lima, ${ }^{105}$ C. Roda, ${ }^{121 a, 121 b}$ D. Roda Dos Santos, ${ }^{29}$ D. Rodriguez, ${ }^{161}$ A. Roe, ${ }^{53}$ S. Roe, ${ }^{29}$ O. Røhne, ${ }^{116}$ V. Rojo, ${ }^{1}$ S. Rolli, ${ }^{160}$ A. Romaniouk, ${ }^{95}$ M. Romano, ${ }^{19 a, 19 b}$ V. M. Romanov,${ }^{64}$ G. Romeo, ${ }^{26}$ E. Romero Adam, ${ }^{166}$ L. Roos, ${ }^{77}$ E. Ros,${ }^{166}$ S. Rosati, ${ }^{131 \mathrm{a}, 131 \mathrm{~b}}$ K. Rosbach, ${ }^{48}$ A. Rose, ${ }^{148}$ M. Rose, ${ }^{75}$ G. A. Rosenbaum, ${ }^{157}$

E. I. Rosenberg, ${ }^{63}$ P. L. Rosendahl,${ }^{13}$ O. Rosenthal, ${ }^{140}$ L. Rosselet ${ }^{48}$ V. Rossetti, ${ }^{11}$ E. Rossi, ${ }^{131 a, 131 b}$ L. P. Rossi ${ }^{49 a}$ M. Rotaru, ${ }^{25 a}$ I. Roth, ${ }^{170}$ J. Rothberg, ${ }^{137}$ D. Rousseau, ${ }^{114}$ C. R. Royon, ${ }^{135}$ A. Rozanov, ${ }^{82}$ Y. Rozen, ${ }^{151}$ X. Ruan, ${ }^{114, b b}$ I. Rubinskiy, ${ }^{41}$ B. Ruckert, ${ }^{97}$ N. Ruckstuhl, ${ }^{104}$ V. I. Rud,${ }^{96}$ C. Rudolph, ${ }^{43}$ F. Rühr, ${ }^{6}$ F. Ruggieri, ${ }^{133 a, 133 b}$ A. Ruiz-Martinez, ${ }^{63}$ V. Rumiantsev, ${ }^{90, a}$ L. Rumyantsev,${ }^{64} \mathrm{~K}$. Runge,${ }^{47}$ Z. Rurikova, ${ }^{47}$ N. A. Rusakovich, ${ }^{64}$ D. R. Rust, ${ }^{60}$ J. P. Rutherfoord, ${ }^{6}$ C. Ruwiedel, ${ }^{14}$ P. Ruzicka, ${ }^{124}$ Y. F. Ryabov, ${ }^{120}$ V. Ryadovikov, ${ }^{127}$ P. Ryan, ${ }^{87}$ M. Rybar, ${ }^{125}$ G. Rybkin,,${ }^{114}$ N. C. Ryder, ${ }^{117}$ S. Rzaeva, ${ }^{10}$ A. F. Saavedra, ${ }^{149}$ I. Sadeh, ${ }^{152}$ H. F-W. Sadrozinski, ${ }^{136}$ R. Sadykov, ${ }^{64}$ F. Safai Tehrani, ${ }^{131 \mathrm{a}, 13 \mathrm{~b}}$ H. Sakamoto, ${ }^{154}$ G. Salamanna, ${ }^{74}$ A. Salamon, ${ }^{132 \mathrm{a}}$ M. Saleem, ${ }^{110}$ D. Salihagic, ${ }^{98}$ A. Salnikov, ${ }^{142}$ J. Salt, ${ }^{166}$ B. M. Salvachua Ferrando, ${ }^{5}$ D. Salvatore,${ }^{36,36 \mathrm{~b}}$ F. Salvatore, ${ }^{148}$ A. Salvucci, ${ }^{103}$ A. Salzburger ${ }^{29}$ D. Sampsonidis, ${ }^{153}$ B. H. Samset, ${ }^{116}$ A. Sanchez,,${ }^{101 \mathrm{a}, 101 \mathrm{~b}}$ H. Sandaker, ${ }^{13}$ H. G. Sander, ${ }^{80}$ M. P. Sanders, ${ }^{97}$ M. Sandhoff, ${ }^{173}$ T. Sandoval, ${ }^{27}$ C. Sandoval, ${ }^{161}$ R. Sandstroem, ${ }^{98}$ S. Sandvoss, ${ }^{173}$ D. P. C. Sankey, ${ }^{128}$ A. Sansoni, ${ }^{46}$ C. Santamarina Rios, ${ }^{84}$ C. Santoni, ${ }^{33}$ R. Santonico $,{ }^{132 a}, 1323 b$ H. Santos,${ }^{123 a}$ J. G. Saraiva, ${ }^{123 a}$ T. Sarangi, ${ }^{171}$ E. Sarkisyan-Grinbaum, ${ }^{7}$ F. Sarri, ${ }^{121 a, 121 b}$ G. Sartisohn, ${ }^{173}$ O. Sasaki ${ }^{65}$ T. Sasaki, ${ }^{65}$ N. Sasao, ${ }^{67}$ I. Satsounkevitch, ${ }^{89}$ G. Sauvage, ${ }^{4}$ E. Sauvan, ${ }^{4}$ J. B. Sauvan, ${ }^{114}$ P. Savard,${ }^{157, e}$ V. Savinov, ${ }^{122}$ D. O. Savu, ${ }^{29}$ L. Sawyer, ${ }^{24,1}$ D. H. Saxon, ${ }^{52}$ L. P. Says,${ }^{33}$ C. Sbarra, ${ }^{19 a}$ A. Sbrizzi, ${ }^{19 a, 19 b}$ O. Scallon, ${ }^{92}$ D. A. Scannicchio, ${ }^{162}$ M. Scarcella, ${ }^{149}$ J. Schaarschmidt, ${ }^{114}$ P. Schacht, ${ }^{98}$ U. Schäfer, ${ }^{80}$ S. Schaepe,${ }^{20}$ S. Schaetzel,${ }^{57 b}$ A. C. Schaffer, ${ }^{114}$ D. Schaile, ${ }^{97}$ R. D. Schamberger, ${ }^{147}$ V. Scharf, ${ }^{57 a}$ V. A. Schegelsky, ${ }^{120}$ D. Scheirich, ${ }^{86}$ M. Schernau, ${ }^{162}$ M. I. Scherzer, ${ }^{34}$ C. Schiavi, ${ }^{49 a, 49 b}$ J. Schieck, ${ }^{97}$ M. Schioppa,${ }^{36 a, 36 b}$ S. Schlenker, ${ }^{29}$ J. L. Schlereth, ${ }^{5}$ E. Schmidt, ${ }^{47}$ K. Schmieden, ${ }^{20}$ C. Schmitt, ${ }^{80}$ S. Schmitt,${ }^{57 b}$ M. Schmitz, ${ }^{20}$ A. Schöning, ${ }^{57 b}$ M. Schott, ${ }^{29}$ D. Schouten, ${ }^{158 a}$ J. Schovancova, ${ }^{124}$ M. Schram, ${ }^{84}$ C. Schroeder, ${ }^{80}$ N. Schroer, ${ }^{57 c}$ S. Schuh,${ }^{29}$ G. Schuler, ${ }^{29}$ J. Schultes,,${ }^{173}$ H.-C. Schultz-Coulon, ${ }^{57 a}$ H. Schulz, ${ }^{15}$ J. W. Schumacher, ${ }^{20}$ M. Schumacher, ${ }^{47}$ B. A. Schumm, ${ }^{136} \mathrm{Ph}$. Schune, ${ }^{135}$ C. Schwanenberger, ${ }^{81}$ A. Schwartzman, ${ }^{142} \mathrm{Ph}$. Schwemling, ${ }^{77}$ R. Schwienhorst, ${ }^{87}$ R. Schwierz ${ }^{43}$ J. Schwindling, ${ }^{135}$ T. Schwindt, ${ }^{20}$ M. Schwoerer, ${ }^{4}$ W. G. Scott, ${ }^{128}$ J. Searcy, ${ }^{113}$ G. Sedov,${ }^{41}$ E. Sedykh, ${ }^{120}$ E. Segura, ${ }^{11}$ S. C. Seidel, ${ }^{102}$ A. Seiden, ${ }^{136}$ F. Seifert, ${ }^{43}$ J. M. Seixas, ${ }^{23 a}$ G. Sekhniaidze, ${ }^{101 a}$ K. E. Selbach, ${ }^{45}$ D. M. Seliverstov, ${ }^{120}$ B. Sellden, ${ }^{145 a}$ 
G. Sellers, ${ }^{72}$ M. Seman,${ }^{143 b}$ N. Semprini-Cesari, ${ }^{19 a, 19 b}$ C. Serfon, ${ }^{97}$ L. Serin,${ }^{114}$ R. Seuster,${ }^{98}$ H. Severini,,${ }^{10}$ M.E. Sevior, ${ }^{85}$ A. Sfyrla, ${ }^{29}$ E. Shabalina, ${ }^{53}$ M. Shamim, ${ }^{113}$ L. Y. Shan, ${ }^{32 a}$ J. T. Shank ${ }^{21}$ Q. T. Shao, ${ }^{85}$ M. Shapiro, ${ }^{14}$ P. B. Shatalov, ${ }^{94}$ L. Shaver, ${ }^{6}$ K. Shaw, ${ }^{163 a, 163 c}$ D. Sherman, ${ }^{174}$ P. Sherwood, ${ }^{76}$ A. Shibata, ${ }^{107}$ H. Shichi, ${ }^{100}$ S. Shimizu, ${ }^{29}$ M. Shimojima, ${ }^{99}$ T. Shin,${ }^{55}$ M. Shiyakova, ${ }^{64}$ A. Shmeleva, ${ }^{93}$ M. J. Shochet, ${ }^{30}$ D. Short, ${ }^{117}$ S. Shrestha, ${ }^{63}$ M. A. Shupe,${ }^{6}$ P. Sicho, ${ }^{124}$ A. Sidoti, ${ }^{131 a, 131 b}$ F. Siegert, ${ }^{47}$ Dj. Sijacki, ${ }^{12 a}$ O. Silbert, ${ }^{170}$ J. Silva, ${ }^{123 a, c}$ Y. Silver, ${ }^{152}$ D. Silverstein, ${ }^{142}$ S. B. Silverstein, ${ }^{145 a}$ V. Simak, ${ }^{126}$ O. Simard, ${ }^{135}$ Lj. Simic, ${ }^{12 a}$ S. Simion, ${ }^{114}$

B. Simmons, ${ }^{76}$ M. Simonyan, ${ }^{35}$ P. Sinervo, ${ }^{157}$ N. B. Sinev, ${ }^{113}$ V. Sipica, ${ }^{140}$ G. Siragusa, ${ }^{172}$ A. Sircar, ${ }^{24}$

A. N. Sisakyan, ${ }^{64}$ S. Yu. Sivoklokov, ${ }^{96}$ J. Sjölin, ${ }^{145 a, 145 b}$ T. B. Sjursen, ${ }^{13}$ L. A. Skinnari, ${ }^{14}$ H. P. Skottowe,${ }^{56}$ K. Skovpen, ${ }^{106}$ P. Skubic, ${ }^{110}$ N. Skvorodnev, ${ }^{22}$ M. Slater, ${ }^{17}$ T. Slavicek, ${ }^{126}$ K. Sliwa, ${ }^{160}$ J. Sloper, ${ }^{29}$ V. Smakhtin, ${ }^{170}$ S. Yu. Smirnov, ${ }^{95}$ L. N. Smirnova, ${ }^{96}$ O. Smirnova ${ }^{78}$ B. C. Smith, ${ }^{56}$ K. M. Smith, ${ }^{52}$ M. Smizanska,${ }^{70}$ K. Smolek, ${ }^{126}$ A. A. Snesarev, ${ }^{93}$ S. W. Snow, ${ }^{81}$ J. Snow, ${ }^{110}$ J. Snuverink, ${ }^{104}$ S. Snyder, ${ }^{24}$ M. Soares, ${ }^{123 a}$ R. Sobie, ${ }^{168, j}$ J. Sodomka, ${ }^{126}$ A. Soffer ${ }^{152}$ C. A. Solans, ${ }^{166}$ M. Solar, ${ }^{126}$ J. Solc, ${ }^{126}$ E. Soldatov, ${ }^{95}$ U. Soldevila, ${ }^{166}$ E. Solfaroli Camillocci, ${ }^{131 a, 131 b}$ A. A. Solodkov, ${ }^{127}$ O. V. Solovyanov, ${ }^{127}$ N. Soni, ${ }^{2}$ V. Sopko, ${ }^{126}$ B. Sopko, ${ }^{126}$ M. Sosebee, ${ }^{7}$ R. Soualah,,${ }^{163 a, 163 c}$ A. Soukharev, ${ }^{106}$ S. Spagnolo,${ }^{71 a, 71 b}$ F. Spanò ${ }^{75}$ R. Spighi, ${ }^{19 a}$ G. Spigo, ${ }^{29}$ F. Spila, ${ }^{131 a, 131 b}$ R. Spiwoks, ${ }^{29}$ M. Spousta, ${ }^{125}$ T. Spreitzer, ${ }^{157}$ B. Spurlock, ${ }^{7}$ R. D. St. Denis, ${ }^{52}$ T. Stahl, ${ }^{140}$ J. Stahlman, ${ }^{119}$ R. Stamen, ${ }^{57 a}$ E. Stanecka, ${ }^{38}$ R. W. Stanek, ${ }^{5}$ C. Stanescu, ${ }^{133 a}$ S. Stapnes, ${ }^{116}$ E. A. Starchenko, ${ }^{127}$ J. Stark, ${ }^{54}$ P. Staroba, ${ }^{124}$ P. Starovoitov, ${ }^{90}$ A. Staude, ${ }^{97}$ P. Stavina, ${ }^{143}$ G. Stavropoulos, ${ }^{14}$ G. Steele, ${ }^{52}$ P. Steinbach, ${ }^{43}$ P. Steinberg, ${ }^{24}$ I. Stekl, ${ }^{126}$ B. Stelzer, ${ }^{141}$ H. J. Stelzer, ${ }^{87}$ O. Stelzer-Chilton, ${ }^{158 a}$ H. Stenzel, ${ }^{51}$ S. Stern,,${ }^{98}$ K. Stevenson, ${ }^{74}$ G. A. Stewart, ${ }^{29}$ J. A. Stillings, ${ }^{20}$ M. C. Stockton, ${ }^{29}$ K. Stoerig,${ }^{47}$ G. Stoicea, ${ }^{25 a}$ S. Stonjek,${ }^{98}$ P. Strachota, ${ }^{125}$ A. R. Stradling, ${ }^{7}$ A. Straessner, ${ }^{43}$ J. Strandberg, ${ }^{146}$ S. Strandberg, ${ }^{145 a, 145 b}$ A. Strandlie, ${ }^{116}$ M. Strang, ${ }^{108}$ E. Strauss, ${ }^{142}$ M. Strauss, ${ }^{110}$ P. Strizenec, ${ }^{143 b}$ R. Ströhmer, ${ }^{172}$ D. M. Strom, ${ }^{113}$ J. A. Strong,${ }^{75, a}$ R. Stroynowski, ${ }^{39}$ J. Strube, ${ }^{128}$ B. Stugu, ${ }^{13}$ I. Stumer, ${ }^{24, a}$ J. Stupak, ${ }^{147}$ P. Sturm, ${ }^{173}$ N. A. Styles, ${ }^{41}$ D. A. Soh, ${ }^{150, s}$ D. Su, ${ }^{142}$ HS. Subramania, ${ }^{2}$ A. Succurro, ${ }^{11}$ Y. Sugaya, ${ }^{115}$ T. Sugimoto, ${ }^{100}$ C. Suhr, ${ }^{105}$ K. Suita,${ }^{66}$ M. Suk, ${ }^{125}$ V. V. Sulin, ${ }^{93}$ S. Sultansoy, ${ }^{3 \mathrm{~d}}$ T. Sumida, ${ }^{67}$ X. Sun, ${ }^{54}$ J. E. Sundermann, ${ }^{47}$ K. Suruliz, ${ }^{138}$ S. Sushkov, ${ }^{11}$ G. Susinno, ${ }^{36 a, 36 b}$ M. R. Sutton, ${ }^{148}$ Y. Suzuki, ${ }^{65}$ Y. Suzuki, ${ }^{66}$ M. Svatos, ${ }^{124}$ Yu. M. Sviridov, ${ }^{127}$ S. Swedish, ${ }^{167}$ I. Sykora, ${ }^{143 a}$ T. Sykora, ${ }^{125}$ B. Szeless, ${ }^{29}$ J. Sánchez, ${ }^{166}$ D. Ta, ${ }^{104}$ K. Tackmann, ${ }^{41}$ A. Taffard, ${ }^{162}$ R. Tafirout, ${ }^{158 a}$ N. Taiblum, ${ }^{152}$ Y. Takahashi,${ }^{100}$ H. Takai, ${ }^{24}$ R. Takashima,${ }^{68}$ H. Takeda, ${ }^{66}$ T. Takeshita, ${ }^{139}$ Y. Takubo, ${ }^{65}$ M. Talby,${ }^{82}$ A. Talyshev, ${ }^{106}$ M. C. Tamsett, ${ }^{24}$ J. Tanaka, ${ }^{154}$ R. Tanaka, ${ }^{114}$ S. Tanaka ${ }^{130}$ S. Tanaka, ${ }^{65}$ Y. Tanaka, ${ }^{99}$ A. J. Tanasijczuk, ${ }^{141}$ K. Tani, ${ }^{66}$ N. Tannoury, ${ }^{82}$ G. P. Tappern,${ }^{29}$

S. Tapprogge ${ }^{80}$ D. Tardif,${ }^{157}$ S. Tarem,${ }^{151}$ F. Tarrade,${ }^{28}$ G. F. Tartarelli, ${ }^{88 a}$ P. Tas, ${ }^{125}$ M. Tasevsky, ${ }^{124}$ E. Tassi, ${ }^{36 a, 36 b}$ M. Tatarkhanov, ${ }^{14}$ Y. Tayalati, ${ }^{134 \mathrm{~d}}$ C. Taylor, ${ }^{76}$ F. E. Taylor, ${ }^{91}$ G. N. Taylor, ${ }^{85}$ W. Taylor, ${ }^{158 b}$ M. Teinturier, ${ }^{114}$ M. Teixeira Dias Castanheira, ${ }^{74}$ P. Teixeira-Dias, ${ }^{75}$ K. K. Temming, ${ }^{47}$ H. Ten Kate, ${ }^{29}$ P. K. Teng, ${ }^{150}$ S. Terada, ${ }^{65}$ K. Terashi, ${ }^{154}$ J. Terron, ${ }^{79}$ M. Testa, ${ }^{46}$ R. J. Teuscher ${ }^{157, j}$ J. Thadome, ${ }^{173}$ J. Therhaag, ${ }^{20}$ T. Theveneaux-Pelzer, ${ }^{77}$ M. Thioye, ${ }^{174}$ S. Thoma,${ }^{47}$ J.P. Thomas, ${ }^{17}$ E. N. Thompson, ${ }^{34}$ P. D. Thompson, ${ }^{17}$ P. D. Thompson,,${ }^{157}$ A. S. Thompson, ${ }^{52}$ E. Thomson, ${ }^{119}$ M. Thomson,${ }^{27}$ R. P. Thun,${ }^{86}$ F. Tian,${ }^{34}$ M. J. Tibbetts,${ }^{14}$ T. Tic, ${ }^{124}$ V. O. Tikhomirov, ${ }^{93}$ Y. A. Tikhonov, ${ }^{106} \mathrm{~S}$. Timoshenko, ${ }^{95} \mathrm{P}$. Tipton, ${ }^{174}$ F. J. Tique Aires Viegas, ${ }^{29} \mathrm{~S}$. Tisserant, ${ }^{82}$ J. Tobias, ${ }^{47} \mathrm{~B}$. Toczek, ${ }^{37} \mathrm{~T}$. Todorov, ${ }^{4} \mathrm{~S}$. Todorova-Nova, ${ }^{160} \mathrm{~B}$. Toggerson, ${ }^{162} \mathrm{~J}$. Tojo, ${ }^{65} \mathrm{~S}$. Tokár, ${ }^{143 \mathrm{a}} \mathrm{K}$. Tokunaga, ${ }^{66}$ K. Tokushuku, ${ }^{65} \mathrm{~K}$. Tollefson, ${ }^{87} \mathrm{M}$. Tomoto, ${ }^{100} \mathrm{~L}$. Tompkins, ${ }^{30} \mathrm{~K}$. Toms, ${ }^{102} \mathrm{G}$. Tong, ${ }^{32 \mathrm{a}}$ A. Tonoyan, ${ }^{13} \mathrm{C}$. Topfel, ${ }^{16}$ N. D. Topilin, ${ }^{64}$ I. Torchiani, ${ }^{29}$ E. Torrence, ${ }^{113}$ H. Torres, ${ }^{77}$ E. Torró Pastor, ${ }^{166}$ J. Toth,${ }^{82, y}$ F. Touchard ${ }^{82}$

D. R. Tovey, ${ }^{138}$ T. Trefzger, ${ }^{172}$ L. Tremblet ${ }^{29}$ A. Tricoli, ${ }^{29}$ I. M. Trigger, ${ }^{158 a}$ S. Trincaz-Duvoid ${ }^{77}$ T. N. Trinh,${ }^{77}$

M. F. Tripiana, ${ }^{69}$ W. Trischuk,${ }^{157}$ A. Trivedi, ${ }^{24, \mathrm{x}}$ B. Trocmé,${ }^{54}$ C. Troncon,${ }^{88 a}$ M. Trottier-McDonald, ${ }^{141}$

M. Trzebinski, ${ }^{38}$ A. Trzupek,${ }^{38}$ C. Tsarouchas, ${ }^{29}$ J. C-L. Tseng, ${ }^{117}$ M. Tsiakiris,${ }^{104}$ P. V. Tsiareshka, ${ }^{89}$ D. Tsionou, ${ }^{4, c c}$ G. Tsipolitis, ${ }^{9}$ V. Tsiskaridze,${ }^{47}$ E. G. Tskhadadze,${ }^{50 a}$ I. I. Tsukerman, ${ }^{94}$ V. Tsulaia,${ }^{14}$ J.-W. Tsung, ${ }^{20}$ S. Tsuno,${ }^{65}$ D. Tsybychev ${ }^{147}$ A. Tua, ${ }^{138}$ A. Tudorache, ${ }^{25 a}$ V. Tudorache,${ }^{25 a}$ J. M. Tuggle,${ }^{30}$ M. Turala,${ }^{38}$ D. Turecek, ${ }^{126}$ I. Turk Cakir, ${ }^{3 \mathrm{e}}$ E. Turlay, ${ }^{104}$ R. Turra ${ }^{88 \mathrm{a}, 88 \mathrm{~b}}$ P. M. Tuts, ${ }^{34}$ A. Tykhonov, ${ }^{73}$ M. Tylmad,${ }^{145 a, 145 \mathrm{~b}}$ M. Tyndel, ${ }^{128}$ G. Tzanakos, ${ }^{8}$ K. Uchida, ${ }^{20}$ I. Ueda, ${ }^{154}$ R. Ueno,${ }^{28}$ M. Ugland, ${ }^{13}$ M. Uhlenbrock, ${ }^{20}$ M. Uhrmacher, ${ }^{53}$ F. Ukegawa, ${ }^{159}$ G. Unal, ${ }^{29}$ D. G. Underwood, ${ }^{5}$ A. Undrus,${ }^{24}$ G. Unel, ${ }^{162}$ Y. Unno, ${ }^{65}$ D. Urbaniec,${ }^{34}$ G. Usai, ${ }^{7}$ L. Vacavant ${ }^{82}$ V. Vacek, ${ }^{126}$ B. Vachon, ${ }^{84}$ S. Vahsen, ${ }^{14}$ J. Valenta, ${ }^{124}$ P. Valente, ${ }^{131 \mathrm{a}}$ S. Valentinetti, ${ }^{19 a, 19 \mathrm{~b}}$ S. Valkar,${ }^{125}$ E. Valladolid Gallego, ${ }^{166}$ S. Vallecorsa, ${ }^{151}$ J. A. Valls Ferrer, ${ }^{166}$ H. van der Graaf, ${ }^{104}$ E. van der Kraaij, ${ }^{104}$

R. Van Der Leeuw, ${ }^{104}$ E. van der Poel, ${ }^{104}$ D. van der Ster, ${ }^{29}$ N. van Eldik, ${ }^{83}$ P. van Gemmeren, ${ }^{5}$ Z. van Kesteren, ${ }^{104}$ I. van Vulpen, ${ }^{104}$ M. Vanadia, ${ }^{98}$ W. Vandelli, ${ }^{29}$ G. Vandoni,${ }^{29}$ A. Vaniachine, ${ }^{5}$ P. Vankov,${ }^{41}$ F. Vannucci, ${ }^{77}$ F. Varela Rodriguez, ${ }^{29}$ R. Vari, ${ }^{131 a}$ D. Varouchas, ${ }^{14}$ A. Vartapetian, ${ }^{7}$ K. E. Varvell, ${ }^{149}$ V. I. Vassilakopoulos, ${ }^{55}$ 
F. Vazeille, ${ }^{33}$ G. Vegni $,{ }^{88 a}, 88 \mathrm{~b}$ J. J. Veillet, ${ }^{114} \mathrm{C}$. Vellidis, ${ }^{8} \mathrm{~F}$. Veloso, ${ }^{123 a}$ R. Veness, ${ }^{29} \mathrm{~S}$. Veneziano, ${ }^{131 \mathrm{a}}$ A. Ventura, ${ }^{71 a, 71 b}$ D. Ventura, ${ }^{137}$ M. Venturi, ${ }^{47}$ N. Venturi, ${ }^{157}$ V. Vercesi, ${ }^{118 a}$ M. Verducci, ${ }^{137}$ W. Verkerke,${ }^{104}$ J. C. Vermeulen, ${ }^{104}$ A. Vest,${ }^{43}$ M. C. Vetterli, ${ }^{141, e}$ I. Vichou, ${ }^{164}$ T. Vickey, ${ }^{144 b, d d}$ O. E. Vickey Boeriu, ${ }^{144 b}$ G. H. A. Viehhauser ${ }^{117}$ S. Viel, ${ }^{167}$ M. Villa, ${ }^{19 a, 19 b}$ M. Villaplana Perez,${ }^{166}$ E. Vilucchi,${ }^{46}$ M. G. Vincter, ${ }^{28}$ E. Vinek, ${ }^{29}$ V. B. Vinogradov ${ }^{64}$ M. Virchaux,${ }^{135, a}$ J. Virzi,${ }^{14}$ O. Vitells, ${ }^{170}$ M. Viti, ${ }^{41}$ I. Vivarelli, ${ }^{47}$ F. Vives Vaque, ${ }^{2}$ S. Vlachos, ${ }^{9}$

D. Vladoiu, ${ }^{97}$ M. Vlasak, ${ }^{126}$ N. Vlasov, ${ }^{20}$ A. Vogel,${ }^{20}$ P. Vokac, ${ }^{126}$ G. Volpi,${ }^{46}$ M. Volpi,${ }^{85}$ G. Volpini, ${ }^{88 a}$ H. von der Schmitt, ${ }^{98}$ J. von Loeben, ${ }^{98}$ H. von Radziewski, ${ }^{47}$ E. von Toerne, ${ }^{20}$ V. Vorobel, ${ }^{125}$ A. P. Vorobiev, ${ }^{127}$ V. Vorwerk, ${ }^{11}$ M. Vos, ${ }^{166}$ R. Voss, ${ }^{29}$ T. T. Voss, ${ }^{173}$ J. H. Vossebeld, ${ }^{72}$ N. Vranjes, ${ }^{12 a}$ M. Vranjes Milosavljevic,,${ }^{104}$ V. Vrba, ${ }^{124}$ M. Vreeswijk, ${ }^{104}$ T. Vu Anh, ${ }^{80}$ R. Vuillermet, ${ }^{29}$ I. Vukotic, ${ }^{114}$ W. Wagner, ${ }^{173}$ P. Wagner, ${ }^{119}$ H. Wahlen, ${ }^{173}$ J. Wakabayashi, ${ }^{100}$ J. Walbersloh, ${ }^{42}$ S. Walch, ${ }^{86}$ J. Walder, ${ }^{70}$ R. Walker, ${ }^{97}$ W. Walkowiak, ${ }^{140}$ R. Wall, ${ }^{174}$ P. Waller, ${ }^{72}$

C. Wang, ${ }^{44}$ H. Wang, ${ }^{171}$ H. Wang, ${ }^{32 b, e e}$ J. Wang, ${ }^{150}$ J. Wang, ${ }^{54}$ J. C. Wang, ${ }^{137}$ R. Wang, ${ }^{102}$ S. M. Wang, ${ }^{150}$ A. Warburton, ${ }^{84}$ C. P. Ward, ${ }^{27}$ M. Warsinsky,${ }^{47}$ P. M. Watkins, ${ }^{17}$ A. T. Watson, ${ }^{17}$ I. J. Watson, ${ }^{149}$ M. F. Watson, ${ }^{17}$ G. Watts, ${ }^{137}$ S. Watts, ${ }^{81}$ A. T. Waugh, ${ }^{149}$ B. M. Waugh,${ }^{76}$ M. Weber, ${ }^{128}$ M. S. Weber, ${ }^{16}$ P. Weber, ${ }^{53}$ A. R. Weidberg, ${ }^{117}$ P. Weigell, ${ }^{98}$ J. Weingarten, ${ }^{53}$ C. Weiser, ${ }^{47}$ H. Wellenstein, ${ }^{22}$ P. S. Wells, ${ }^{29}$ M. Wen,${ }^{46}$ T. Wenaus, ${ }^{24}$ S. Wendler,${ }^{122}$ Z. Weng, ${ }^{150, \mathrm{~s}}$ T. Wengler, ${ }^{29} \mathrm{~S}$. Wenig, ${ }^{29} \mathrm{~N}$. Wermes,${ }^{20} \mathrm{M}$. Werner, ${ }^{47} \mathrm{P}$. Werner, ${ }^{29} \mathrm{M}$. Werth,${ }^{162} \mathrm{M}$. Wessels, ${ }^{57 \mathrm{a}}$ C. Weydert, ${ }^{54}$ K. Whalen, ${ }^{28}$ S. J. Wheeler-Ellis, ${ }^{162}$ S. P. Whitaker, ${ }^{21}$ A. White, ${ }^{7}$ M. J. White, ${ }^{85}$ S. R. Whitehead,${ }^{117}$ D. Whiteson, ${ }^{162}$ D. Whittington, ${ }^{60}$ D. Wicke, ${ }^{173}$ F. J. Wickens, ${ }^{128}$ W. Wiedenmann, ${ }^{171}$ M. Wielers, ${ }^{128}$ P. Wienemann, ${ }^{20}$ C. Wiglesworth, ${ }^{74}$ L. A. M. Wiik,${ }^{47}$ P. A. Wijeratne, ${ }^{76}$ A. Wildauer, ${ }^{166}$ M. A. Wildt, ${ }^{41, p}$ I. Wilhelm, ${ }^{125}$ H. G. Wilkens, ${ }^{29}$ J.Z. Will,${ }^{97}$ E. Williams, ${ }^{34}$ H. H. Williams, ${ }^{119}$ W. Willis, ${ }^{34}$ S. Willocq, ${ }^{83}$ J. A. Wilson, ${ }^{17}$ M. G. Wilson, ${ }^{142}$ A. Wilson, ${ }^{86}$ I. Wingerter-Seez, ${ }^{4}$ S. Winkelmann, ${ }^{47}$ F. Winklmeier, ${ }^{29}$ M. Wittgen, ${ }^{142}$ M. W. Wolter, ${ }^{38}$ H. Wolters, ${ }^{123 a, h}$ W. C. Wong, ${ }^{40}$ G. Wooden, ${ }^{86}$ B. K. Wosiek ${ }^{38}$ J. Wotschack,${ }^{29}$ M. J. Woudstra, ${ }^{83}$ K. W. Wozniak, ${ }^{38}$ K. Wraight, ${ }^{52}$ C. Wright, ${ }^{52}$ M. Wright, ${ }^{52}$ B. Wrona,${ }^{72}$ S. L. Wu, ${ }^{171}$ X. Wu, ${ }^{48}$ Y. Wu, ${ }^{32 b, f f}$ E. Wulf, ${ }^{34}$ R. Wunstorf, ${ }^{42}$ B. M. Wynne, ${ }^{45}$ S. Xella,${ }^{35}$ M. Xiao, ${ }^{135}$ S. Xie, ${ }^{47}$ Y. Xie,${ }^{32 a}$ C. Xu, ${ }^{32 b, g g}$ D. Xu, ${ }^{138}$ G. Xu, ${ }^{32 a}$ B. Yabsley, ${ }^{149}$ S. Yacoob ${ }^{144 b}$ M. Yamada ${ }^{65}$ H. Yamaguchi, ${ }^{154}$ A. Yamamoto, ${ }^{65}$ K. Yamamoto, ${ }^{63}$ S. Yamamoto, ${ }^{154}$ T. Yamamura, ${ }^{154}$ T. Yamanaka, ${ }^{154}$ J. Yamaoka, ${ }^{44}$ T. Yamazaki, ${ }^{154}$ Y. Yamazaki, ${ }^{66}$ Z. Yan, ${ }^{21}$ H. Yang, ${ }^{86}$ U. K. Yang, ${ }^{81}$ Y. Yang, ${ }^{60}$ Y. Yang, ${ }^{32 \mathrm{a}}$ Z. Yang, ${ }^{145 a, 145 b}$ S. Yanush, ${ }^{90}$ Y. Yasu, ${ }^{65}$ G. V. Ybeles Smit,${ }^{129}$ J. Ye, ${ }^{39}$ S. Ye,${ }^{24}$ M. Yilmaz ${ }^{3 c}$ R. Yoosoofmiya, ${ }^{122}$ K. Yorita, ${ }^{169}$ R. Yoshida ${ }^{5}$ C. Young, ${ }^{142}$ S. Youssef, ${ }^{21}$ D. Yu, ${ }^{24}$ J. Yu, ${ }^{7}$ J Yu, ${ }^{111}$ L. Yuan, ${ }^{32 a, h h}$ A. Yurkewicz, ${ }^{105}$ B. Zabinski, ${ }^{38}$ V. G. Zaets, ${ }^{127}$ R. Zaidan, ${ }^{62}$ A. M. Zaitsev, ${ }^{127}$ Z. Zajacova, ${ }^{29}$ L. Zanello, ${ }^{131 a, 131 b}$ P. Zarzhitsky, ${ }^{39}$ A. Zaytsev, ${ }^{106}$ C. Zeitnitz, ${ }^{173}$ M. Zeller, ${ }^{174}$ M. Zeman, ${ }^{124}$ A. Zemla, ${ }^{38}$ C. Zendler, ${ }^{20}$ O. Zenin, ${ }^{127}$

T. Ženiš, ${ }^{143 a}$ Z. Zenonos, ${ }^{121 \mathrm{a}, 121 \mathrm{~b}}$ S. Zenz, ${ }^{14}$ D. Zerwas, ${ }^{114}$ G. Zevi della Porta, ${ }^{56}$ Z. Zhan, ${ }^{32 \mathrm{~d}}$ D. Zhang, ${ }^{32 \mathrm{~b}, e e}$ H. Zhang, ${ }^{87}$ J. Zhang, ${ }^{5}$ X. Zhang, ${ }^{32 \mathrm{~d}}$ Z. Zhang, ${ }^{114}$ L. Zhao, ${ }^{107}$ T. Zhao, ${ }^{137}$ Z. Zhao, ${ }^{32 \mathrm{~b}}$ A. Zhemchugov, ${ }^{64}$ S. Zheng, ${ }^{32 \mathrm{a}}$ J. Zhong, ${ }^{117}$ B. Zhou, ${ }^{86}$ N. Zhou, ${ }^{162}$ Y. Zhou, ${ }^{150}$ C. G. Zhu, ${ }^{32 \mathrm{~d}}$ H. Zhu, ${ }^{41}$ J. Zhu, ${ }^{86}$ Y. Zhu, ${ }^{32 \mathrm{~b}}$ X. Zhuang, ${ }^{97}$

V. Zhuravlov, ${ }^{98}$ D. Zieminska, ${ }^{60}$ R. Zimmermann, ${ }^{20} \mathrm{~S}$. Zimmermann, ${ }^{20} \mathrm{~S}$. Zimmermann, ${ }^{47} \mathrm{M}$. Ziolkowski, ${ }^{140}$ R. Zitoun, ${ }^{4}$ L. Živković, ${ }^{34}$ V. V. Zmouchko, ${ }^{127, a}$ G. Zobernig, ${ }^{171}$ A. Zoccoli, ${ }^{19 a, 19 b}$ Y. Zolnierowski, ${ }^{4}$ A. Zsenei, ${ }^{29}$ M. zur Nedden, ${ }^{15}$ V. Zutshi, ${ }^{105}$ and L. Zwalinski ${ }^{29}$

(ATLAS Collaboration)

\footnotetext{
${ }^{1}$ University at Albany, Albany, New York, USA

${ }^{2}$ Department of Physics, University of Alberta, Edmonton AB, Canada

${ }^{3 a}$ Department of Physics, Ankara University, Ankara, Turkey

${ }^{3 \mathrm{~b}}$ Department of Physics, Dumlupinar University, Kutahya, Turkey

${ }^{3 c}$ Department of Physics, Gazi University, Ankara, Turkey

${ }^{3 \mathrm{~d}}$ Division of Physics, TOBB University of Economics and Technology, Ankara, Turkey

${ }^{3 e}$ Turkish Atomic Energy Authority, Ankara, Turkey

${ }^{4} L A P P, C N R S / I N 2 P 3$ and Université de Savoie, Annecy-le-Vieux, France

${ }^{5}$ High Energy Physics Division, Argonne National Laboratory, Argonne, Illinois, USA

${ }^{6}$ Department of Physics, University of Arizona, Tucson, Arizona, USA

${ }^{7}$ Department of Physics, The University of Texas at Arlington, Arlington, Texas, USA

${ }^{8}$ Physics Department, University of Athens, Athens, Greece

${ }^{9}$ Physics Department, National Technical University of Athens, Zografou, Greece

${ }^{10}$ Institute of Physics, Azerbaijan Academy of Sciences, Baku, Azerbaijan

${ }^{11}$ Institut de Física d'Altes Energies and Departament de Física de la Universitat Autònoma de Barcelona and ICREA, Barcelona, Spain
} 
${ }^{12 a}$ Institute of Physics, University of Belgrade, Belgrade, Serbia

${ }^{12 \mathrm{~b}}$ Vinca Institute of Nuclear Sciences, Belgrade, Serbia

${ }^{13}$ Department for Physics and Technology, University of Bergen, Bergen, Norway

${ }^{14}$ Physics Division, Lawrence Berkeley National Laboratory and University of California, Berkeley, California, USA

${ }^{15}$ Department of Physics, Humboldt University, Berlin, Germany

${ }^{16}$ Albert Einstein Center for Fundamental Physics and Laboratory for High Energy Physics, University of Bern, Bern, Switzerland

${ }^{17}$ School of Physics and Astronomy, University of Birmingham, Birmingham, United Kingdom

${ }^{18 a}$ Department of Physics, Bogazici University, Istanbul, Turkey

${ }^{18 \mathrm{~b}}$ Division of Physics, Dogus University, Istanbul, Turkey

${ }^{18 c}$ Department of Physics Engineering, Gaziantep University, Gaziantep, Turkey

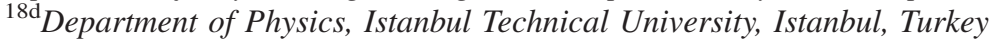

${ }^{19 a}$ INFN Sezione di Bologna, Italy

${ }^{19 \mathrm{~b}}$ Dipartimento di Fisica, Università di Bologna, Bologna, Italy

${ }^{20}$ Physikalisches Institut, University of Bonn, Bonn, Germany

${ }^{21}$ Department of Physics, Boston University, Boston, Massachusetts, USA

${ }^{22}$ Department of Physics, Brandeis University, Waltham, Massachusetts, USA

${ }^{23 a}$ Universidade Federal do Rio De Janeiro COPPE/EE/IF, Rio de Janeiro, Brazil

${ }^{23 \mathrm{~b}}$ Federal University of Juiz de Fora (UFJF), Juiz de Fora, Brazil

${ }^{23 \mathrm{c}}$ Federal University of Sao Joao del Rei (UFSJ), Sao Joao del Rei, Brazil

${ }^{23 \mathrm{~d}}$ Instituto de Fisica, Universidade de Sao Paulo, Sao Paulo, Brazil

${ }^{24}$ Physics Department, Brookhaven National Laboratory, Upton, New York, USA

${ }^{25 \mathrm{a}}$ National Institute of Physics and Nuclear Engineering, Bucharest, Romania

${ }^{25 \mathrm{~b}}$ University Politehnica Bucharest, Bucharest, Romania

${ }^{25 c}$ West University in Timisoara, Timisoara, Romania

${ }^{26}$ Departamento de Física, Universidad de Buenos Aires, Buenos Aires, Argentina

${ }^{27}$ Cavendish Laboratory, University of Cambridge, Cambridge, United Kingdom

${ }^{28}$ Department of Physics, Carleton University, Ottawa ON, Canada

${ }^{29}$ CERN, Geneva, Switzerland

${ }^{30}$ Enrico Fermi Institute, University of Chicago, Chicago, Illinois, USA

${ }^{31 a}$ Departamento de Fisica, Pontificia Universidad Católica de Chile, Santiago, Chile

${ }^{31 \mathrm{~b}}$ Departamento de Física, Universidad Técnica Federico Santa María, Valparaíso, Chile

${ }^{32 \mathrm{a}}$ Institute of High Energy Physics, Chinese Academy of Sciences, Beijing, China

${ }^{32 \mathrm{~b}}$ Department of Modern Physics, University of Science and Technology of China, Anhui, China

${ }^{32 \mathrm{c}}$ Department of Physics, Nanjing University, Jiangsu, China

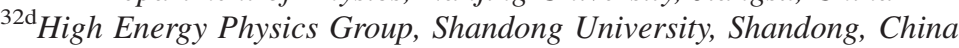

${ }^{33}$ Laboratoire de Physique Corpusculaire, Clermont Université and Université Blaise Pascal and CNRS/IN2P3,

Aubiere Cedex, France

${ }^{34}$ Nevis Laboratory, Columbia University, Irvington, New York, USA

${ }^{35}$ Niels Bohr Institute, University of Copenhagen, Kobenhavn, Denmark

${ }^{36 a}$ INFN Gruppo Collegato di Cosenza, Italy

${ }^{36 \mathrm{~b}}$ Dipartimento di Fisica, Università della Calabria, Arcavata di Rende, Italy

${ }^{37}$ AGH-University of Science and Technology,Faculty of Physics and Applied Computer Science, Krakow, Poland

${ }^{38}$ The Henryk Niewodniczanski Institute of Nuclear Physics, Polish Academy of Sciences, Krakow, Poland

${ }^{39}$ Physics Department, Southern Methodist University, Dallas, Texas, USA

${ }^{40}$ Physics Department, University of Texas at Dallas, Richardson, Texas, USA

${ }^{41}$ DESY, Hamburg and Zeuthen, Germany

${ }^{42}$ Institut für Experimentelle Physik IV, Technische Universität Dortmund, Dortmund, Germany

${ }^{43}$ Institut für Kern- und Teilchenphysik, Technical University Dresden, Dresden, Germany

${ }^{44}$ Department of Physics, Duke University, Durham, North Carolina, USA

${ }^{45}$ SUPA-School of Physics and Astronomy, University of Edinburgh, Edinburgh, United Kingdom

${ }^{46}$ INFN Laboratori Nazionali di Frascati, Frascati, Italy

${ }^{47}$ Fakultät für Mathematik und Physik, Albert-Ludwigs-Universität, Freiburg i.Br., Germany

${ }^{48}$ Section de Physique, Université de Genève, Geneva, Switzerland

${ }^{49}$ INFN Sezione di Genova, Italy

${ }^{49 b}$ Dipartimento di Fisica, Università di Genova, Genova, Italy

${ }^{50 a}$ E.Andronikashvili Institute of Physics, Georgian Academy of Sciences, Tbilisi, Georgia

${ }^{50 b}$ High Energy Physics Institute, Tbilisi State University, Tbilisi, Georgia

${ }^{51}$ II Physikalisches Institut, Justus-Liebig-Universität Giessen, Giessen, Germany

${ }^{52}$ SUPA-School of Physics and Astronomy, University of Glasgow, Glasgow, United Kingdom

${ }^{53}$ II Physikalisches Institut, Georg-August-Universität, Göttingen, Germany 
${ }^{54}$ Laboratoire de Physique Subatomique et de Cosmologie, Université Joseph Fourier and CNRS/IN2P3 and Institut National Polytechnique de Grenoble, Grenoble, France

${ }^{55}$ Department of Physics, Hampton University, Hampton, Virginia, USA

${ }^{56}$ Laboratory for Particle Physics and Cosmology, Harvard University, Cambridge, Massachusetts, USA

${ }^{57}$ Kirchhoff-Institut für Physik, Ruprecht-Karls-Universität Heidelberg, Heidelberg, Germany

${ }^{57 b}$ Physikalisches Institut, Ruprecht-Karls-Universität Heidelberg, Heidelberg, Germany

${ }^{57 c}$ ZITI Institut für technische Informatik, Ruprecht-Karls-Universität Heidelberg, Mannheim, Germany

${ }^{58}$ Faculty of Science, Hiroshima University, Hiroshima, Japan

${ }^{59}$ Faculty of Applied Information Science, Hiroshima Institute of Technology, Hiroshima, Japan

${ }^{60}$ Department of Physics, Indiana University, Bloomington, Indiana, USA

${ }^{61}$ Institut für Astro- und Teilchenphysik, Leopold-Franzens-Universität, Innsbruck, Austria

${ }^{62}$ University of Iowa, Iowa City, Iowa, USA

${ }^{63}$ Department of Physics and Astronomy, Iowa State University, Ames, Iowa, USA

${ }^{64}$ Joint Institute for Nuclear Research, JINR Dubna, Dubna, Russia

${ }^{65}$ KEK, High Energy Accelerator Research Organization, Tsukuba, Japan

${ }^{66}$ Graduate School of Science, Kobe University, Kobe, Japan

${ }^{67}$ Faculty of Science, Kyoto University, Kyoto, Japan

${ }^{68}$ Kyoto University of Education, Kyoto, Japan

${ }^{69}$ Instituto de Física La Plata, Universidad Nacional de La Plata and CONICET, La Plata, Argentina

${ }^{70}$ Physics Department, Lancaster University, Lancaster, United Kingdom

${ }^{71 \mathrm{a}}$ INFN Sezione di Lecce, Italy

${ }^{71 \mathrm{~b}}$ Dipartimento di Fisica, Università del Salento, Lecce, Italy

${ }^{72}$ Oliver Lodge Laboratory, University of Liverpool, Liverpool, United Kingdom

${ }^{73}$ Department of Physics, Jožef Stefan Institute and University of Ljubljana, Ljubljana, Slovenia

${ }^{74}$ School of Physics and Astronomy, Queen Mary University of London, London, United Kingdom

${ }^{75}$ Department of Physics, Royal Holloway University of London, Surrey, United Kingdom

${ }^{76}$ Department of Physics and Astronomy, University College London, London, United Kingdom

${ }^{77}$ Laboratoire de Physique Nucléaire et de Hautes Energies, UPMC and Université Paris-Diderot and CNRS/IN2P3, Paris, France

${ }^{78}$ Fysiska institutionen, Lunds universitet, Lund, Sweden

${ }^{79}$ Departamento de Fisica Teorica C-15, Universidad Autonoma de Madrid, Madrid, Spain

${ }^{80}$ Institut für Physik, Universität Mainz, Mainz, Germany

${ }^{81}$ School of Physics and Astronomy, University of Manchester, Manchester, United Kingdom

${ }^{82}$ CPPM, Aix-Marseille Université and CNRS/IN2P3, Marseille, France

${ }^{83}$ Department of Physics, University of Massachusetts, Amherst, Massachusetts, USA

${ }^{84}$ Department of Physics, McGill University, Montreal QC, Canada

${ }^{85}$ School of Physics, University of Melbourne, Victoria, Australia

${ }^{86}$ Department of Physics, The University of Michigan, Ann Arbor, Michigan, USA

${ }^{87}$ Department of Physics and Astronomy, Michigan State University, East Lansing, Michigan, USA

${ }^{88}$ INFN Sezione di Milano, Italy

${ }^{88 \mathrm{~b}}$ Dipartimento di Fisica, Università di Milano, Milano, Italy

${ }^{89}$ B.I. Stepanov Institute of Physics, National Academy of Sciences of Belarus, Minsk, Republic of Belarus

${ }^{90}$ National Scientific and Educational Centre for Particle and High Energy Physics, Minsk, Republic of Belarus

${ }^{91}$ Department of Physics, Massachusetts Institute of Technology, Cambridge, Massachusetts, USA

${ }^{92}$ Group of Particle Physics, University of Montreal, Montreal QC, Canada

${ }^{93}$ P.N. Lebedev Institute of Physics, Academy of Sciences, Moscow, Russia

${ }^{94}$ Institute for Theoretical and Experimental Physics (ITEP), Moscow, Russia

${ }^{95}$ Moscow Engineering and Physics Institute (MEPhI), Moscow, Russia

${ }^{96}$ Skobeltsyn Institute of Nuclear Physics, Lomonosov Moscow State University, Moscow, Russia

${ }^{97}$ Fakultät für Physik, Ludwig-Maximilians-Universität München, München, Germany

${ }^{98}$ Max-Planck-Institut für Physik (Werner-Heisenberg-Institut), München, Germany

${ }^{99}$ Nagasaki Institute of Applied Science, Nagasaki, Japan

${ }^{100}$ Graduate School of Science, Nagoya University, Nagoya, Japan

${ }^{101 a}$ INFN Sezione di Napoli, Italy

${ }^{101 \mathrm{~b}}$ Dipartimento di Scienze Fisiche, Università di Napoli, Napoli, Italy

${ }^{102}$ Department of Physics and Astronomy, University of New Mexico, Albuquerque New Mexico, USA

${ }^{103}$ Institute for Mathematics, Astrophysics and Particle Physics, Radboud University Nijmegen/Nikhef, Nijmegen, Netherlands

${ }^{104}$ Nikhef National Institute for Subatomic Physics and University of Amsterdam, Amsterdam, Netherlands

${ }^{105}$ Department of Physics, Northern Illinois University, DeKalb, Illinois, USA

${ }^{106}$ Budker Institute of Nuclear Physics (BINP), Novosibirsk, Russia

${ }^{107}$ Department of Physics, New York University, New York, New York, USA

${ }^{108}$ Ohio State University, Columbus, Ohio, USA 
${ }^{109}$ Faculty of Science, Okayama University, Okayama, Japan

${ }^{110}$ Homer L. Dodge Department of Physics and Astronomy, University of Oklahoma, Norman, Oklahoma, USA

${ }^{111}$ Department of Physics, Oklahoma State University, Stillwater, Oklahoma, USA

${ }^{112}$ Palacký University, RCPTM, Olomouc, Czech Republic

${ }^{113}$ Center for High Energy Physics, University of Oregon, Eugene, Oregon, USA

${ }^{114}$ LAL, Univ. Paris-Sud and CNRS/IN2P3, Orsay, France

${ }^{115}$ Graduate School of Science, Osaka University, Osaka, Japan

${ }^{116}$ Department of Physics, University of Oslo, Oslo, Norway

${ }^{117}$ Department of Physics, Oxford University, Oxford, United Kingdom

${ }^{118 a}$ INFN Sezione di Pavia, Italy

${ }^{118 b}$ Dipartimento di Fisica Nucleare e Teorica, Università di Pavia, Pavia, Italy

${ }^{119}$ Department of Physics, University of Pennsylvania, Philadelphia, Pennsylvania, USA

${ }^{120}$ Petersburg Nuclear Physics Institute, Gatchina, Russia

${ }^{121 a}$ INFN Sezione di Pisa, Italy

${ }^{121 \mathrm{~b}}$ Dipartimento di Fisica E. Fermi, Università di Pisa, Pisa, Italy

${ }^{122}$ Department of Physics and Astronomy, University of Pittsburgh, Pittsburgh, Pennsylvania, USA

${ }^{123 a}$ Laboratorio de Instrumentacao e Fisica Experimental de Particulas-LIP, Lisboa, Portugal

${ }^{123 \mathrm{~b}}$ Departamento de Fisica Teorica y del Cosmos and CAFPE, Universidad de Granada, Granada, Portugal

${ }^{124}$ Institute of Physics, Academy of Sciences of the Czech Republic, Praha, Czech Republic

${ }^{125}$ Faculty of Mathematics and Physics, Charles University in Prague, Praha, Czech Republic

${ }^{126}$ Czech Technical University in Prague, Praha, Czech Republic

${ }^{127}$ State Research Center Institute for High Energy Physics, Protvino, Russia

${ }^{128}$ Particle Physics Department, Rutherford Appleton Laboratory, Didcot, United Kingdom

${ }^{129}$ Physics Department, University of Regina, Regina SK, Canada

${ }^{130}$ Ritsumeikan University, Kusatsu, Shiga, Japan

${ }^{131 \mathrm{a} I N F N}$ Sezione di Roma I, Italy

${ }^{131 \mathrm{~b}}$ Dipartimento di Fisica, Università La Sapienza, Roma, Italy

${ }^{132 a}$ INFN Sezione di Roma Tor Vergata, Italy

${ }^{1323 b}$ Dipartimento di Fisica, Università di Roma Tor Vergata, Roma, Italy

${ }^{133 a}$ INFN Sezione di Roma Tre, Italy

${ }^{133 \mathrm{~b}}$ Dipartimento di Fisica, Università Roma Tre, Roma, Italy

${ }^{134 a}$ Faculté des Sciences Ain Chock, Réseau Universitaire de Physique des Hautes Energies-Université Hassan II, Casablanca, Morocco

${ }^{134 b}$ Centre National de l'Energie des Sciences Techniques Nucleaires, Rabat, Morocco

${ }^{134 c}$ Université Cadi Ayyad, Faculté des sciences Semlalia Département de Physique, B.P. 2390 Marrakech 40000, Morocco

${ }^{134 \mathrm{~d}}$ Faculté des Sciences, Université Mohamed Premier and LPTPM, Oujda, Morocco

${ }^{134 \mathrm{e}}$ Faculté des Sciences, Université Mohammed V, Rabat, Morocco

${ }^{135}$ DSM/IRFU (Institut de Recherches sur les Lois Fondamentales de l'Univers),

CEA Saclay (Commissariat a l'Energie Atomique), Gif-sur-Yvette, France

${ }^{136}$ Santa Cruz, Institute for Particle Physics, University of California Santa Cruz, Santa Cruz, California, USA

${ }^{137}$ Department of Physics, University of Washington, Seattle, Washington, USA

${ }^{138}$ Department of Physics and Astronomy, University of Sheffield, Sheffield, United Kingdom

${ }^{139}$ Department of Physics, Shinshu University, Nagano, Japan

${ }^{140}$ Fachbereich Physik, Universität Siegen, Siegen, Germany

${ }^{141}$ Department of Physics, Simon Fraser University, Burnaby BC, Canada

${ }^{142}$ SLAC National Accelerator Laboratory, Stanford, California, USA

${ }^{143 a}$ Faculty of Mathematics, Physics \& Informatics, Comenius University, Bratislava, Slovak Republic

${ }^{143 b}$ Department of Subnuclear Physics, Institute of Experimental Physics of the Slovak Academy of Sciences, Kosice, Slovak Republic

${ }^{144 a}$ Department of Physics, University of Johannesburg, Johannesburg, South Africa

${ }^{144 \mathrm{~b}}$ School of Physics, University of the Witwatersrand, Johannesburg, South Africa

${ }^{145}$ Department of Physics, Stockholm University, Sweden

${ }^{145 \mathrm{~b}}$ The Oskar Klein Centre, Stockholm, Sweden

${ }^{146}$ Physics Department, Royal Institute of Technology, Stockholm, Sweden

${ }^{147}$ Department of Physics and Astronomy, Stony Brook University, Stony Brook, New York, USA

${ }^{148}$ Department of Physics and Astronomy, University of Sussex, Brighton, United Kingdom

${ }^{149}$ School of Physics, University of Sydney, Sydney, Australia

${ }^{150}$ Institute of Physics, Academia Sinica, Taipei, Taiwan

${ }^{151}$ Department of Physics, Technion: Israel Inst. of Technology, Haifa, Israel

${ }^{152}$ Raymond and Beverly Sackler School of Physics and Astronomy, Tel Aviv University, Tel Aviv, Israel

${ }^{153}$ Department of Physics, Aristotle University of Thessaloniki, Thessaloniki, Greece

${ }^{154}$ International Center for Elementary Particle Physics and Department of Physics, The University of Tokyo, Tokyo, Japan 
${ }^{155}$ Graduate School of Science and Technology, Tokyo Metropolitan University, Tokyo, Japan

${ }^{156}$ Department of Physics, Tokyo Institute of Technology, Tokyo, Japan

${ }^{157}$ Department of Physics, University of Toronto, Toronto ON, Canada

${ }^{158}$ TRIUMF, Vancouver BC, Canada

${ }^{158 b}$ Department of Physics and Astronomy, York University, Toronto ON, Canada

${ }^{159}$ Institute of Pure and Applied Sciences, University of Tsukuba, 1-1-1 Tennodai,Tsukuba, Ibaraki 305-8571, Japan

${ }^{160}$ Science and Technology Center, Tufts University, Medford, Massachusetts, USA

${ }^{161}$ Centro de Investigaciones, Universidad Antonio Narino, Bogota, Colombia

${ }^{162}$ Department of Physics and Astronomy, University of California Irvine, Irvine, California, USA

${ }^{163 a}$ INFN Gruppo Collegato di Udine, Italy

${ }^{163 \mathrm{~b}}$ ICTP, Trieste, Italy

${ }^{163 \mathrm{c}}$ Dipartimento di Chimica, Fisica e Ambiente, Università di Udine, Udine, Italy

${ }^{164}$ Department of Physics, University of Illinois, Urbana, Illinois, USA

${ }^{165}$ Department of Physics and Astronomy, University of Uppsala, Uppsala, Sweden

${ }^{166}$ Instituto de Física Corpuscular (IFIC) and Departamento de Física Atómica, Molecular y Nuclear and Departamento de Ingeniería Electrónica and Instituto de Microelectrónica de Barcelona (IMB-CNM), University of Valencia and CSIC, Valencia, Spain

${ }^{167}$ Department of Physics, University of British Columbia, Vancouver BC, Canada

${ }^{168}$ Department of Physics and Astronomy, University of Victoria, Victoria BC, Canada

${ }^{169}$ Waseda University, Tokyo, Japan

${ }^{170}$ Department of Particle Physics, The Weizmann Institute of Science, Rehovot, Israel

${ }^{171}$ Department of Physics, University of Wisconsin, Madison, Wisconsin, USA

${ }^{172}$ Fakultät für Physik und Astronomie, Julius-Maximilians-Universität, Würzburg, Germany

${ }^{173}$ Fachbereich C Physik, Bergische Universität Wuppertal, Wuppertal, Germany

${ }^{174}$ Department of Physics, Yale University, New Haven, Connecticut, USA

${ }^{175}$ Yerevan Physics Institute, Yerevan, Armenia

${ }^{176}$ Domaine scientifique de la Doua, Centre de Calcul CNRS/IN2P3, Villeurbanne Cedex, France

${ }^{\mathrm{a}}$ Deceased.

${ }^{\mathrm{b}}$ Also at Laboratorio de Instrumentacao e Fisica Experimental de Particulas-LIP, Lisboa, Portugal.

${ }^{\mathrm{c}}$ Also at Faculdade de Ciencias and CFNUL, Universidade de Lisboa, Lisboa, Portugal.

${ }^{\mathrm{d}}$ Also at Particle Physics Department, Rutherford Appleton Laboratory, Didcot, United Kingdom.

${ }^{\mathrm{e}}$ Also at TRIUMF, Vancouver BC, Canada.

${ }^{\mathrm{f}}$ Also at Department of Physics, California State University, Fresno, CA, USA.

${ }^{\mathrm{g}}$ Also at Fermilab, Batavia, IL, USA.

${ }^{\mathrm{h}}$ Also at Department of Physics, University of Coimbra, Coimbra, Portugal.

${ }^{\mathrm{i}}$ Also at Università di Napoli Parthenope, Napoli, Italy.

${ }^{\mathrm{j}}$ Also at Institute of Particle Physics (IPP), Canada.

${ }^{\mathrm{k}}$ Also at Department of Physics, Middle East Technical University, Ankara, Turkey.

${ }^{1}$ Also at Louisiana Tech University, Ruston, LA, USA.

${ }^{\mathrm{m}}$ Also at Department of Physics and Astronomy, University College London, London, United Kingdom.

${ }^{\mathrm{n}}$ Also at Group of Particle Physics, University of Montreal, Montreal QC, Canada.

${ }^{\circ}$ Also at Institute of Physics, Azerbaijan Academy of Sciences, Baku, Azerbaijan.

${ }^{\mathrm{p}}$ Also at Institut für Experimentalphysik, Universität Hamburg, Hamburg, Germany.

${ }^{\mathrm{q}}$ Also at Manhattan College, New York, NY, USA.

${ }^{\mathrm{r}}$ Also at CPPM, Aix-Marseille Université and CNRS/IN2P3, Marseille, France.

${ }^{\mathrm{s}}$ Also at School of Physics and Engineering, Sun Yat-sen University, Guanzhou, China.

${ }^{t}$ Also at Academia Sinica Grid Computing, Institute of Physics, Academia Sinica, Taipei, Taiwan.

${ }^{\mathrm{u}}$ Also at High Energy Physics Group, Shandong University, Shandong, China.

${ }^{v}$ Also at Section de Physique, Université de Genève, Geneva, Switzerland.

${ }^{\mathrm{w}}$ Also at Departamento de Fisica, Universidade de Minho, Braga, Portugal.

${ }^{\mathrm{x}}$ Also at Department of Physics and Astronomy, University of South Carolina, Columbia, SC, USA.

${ }^{y}$ Also at KFKI Research Institute for Particle and Nuclear Physics, Budapest, Hungary.

${ }^{z}$ Also at California Institute of Technology, Pasadena, CA, USA.

${ }^{\text {aa } A l s o ~ a t ~ I n s t i t u t e ~ o f ~ P h y s i c s, ~ J a g i e l l o n i a n ~ U n i v e r s i t y, ~ K r a k o w, ~ P o l a n d ~}$

${ }^{\mathrm{bb}}$ Also at Institute of High Energy Physics, Chinese Academy of Sciences, Beijing, China.

${ }^{\mathrm{cc}}$ Also at Department of Physics and Astronomy, University of Sheffield, Sheffield, United Kingdom.

${ }^{\mathrm{dd}}$ Also at Department of Physics, Oxford University, Oxford, United Kingdom.

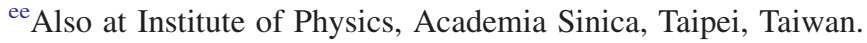


${ }^{\mathrm{ff}}$ Also at Department of Physics, The University of Michigan, Ann Arbor, MI, USA.

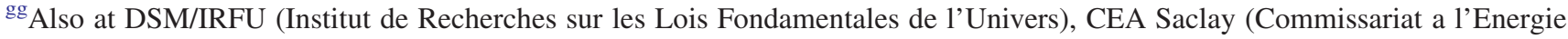
Atomique), Gif-sur-Yvette, France.

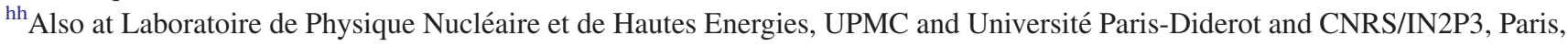
France. 